\title{
Supporting Information: Mechanistic Photochemistry of Methyl-4-Hydroxycinnamate Chromophore and Its One-Water Complexes: Insights from MS-CASPT2 Study
}

\author{
Xiao-Ying Xie, Chun-Xiang Li, Qiu Fang, and Ganglong Cui ${ }^{\star}$ \\ Key Laboratory of Theoretical and Computational Photochemistry, Ministry of Education, \\ College of Chemistry, Beijing Normal University, Beijing 100875, China \\ Email: ganglong.cui@bnu.edu.cn
}

\section{Table of Contents}

1. Geometric Structures and Energy of OMpCA and Its One-Water Complexes

2. Active Orbitals used in CASSCF and MS-CASPT2 Computations

3. Molecular Orbitals Involved in Vertical Electronic Transitions

4. Vertical Excitation Energies at the Franck-Condon Points

5. Cartesian Coordinates of Optimized Structures and Paths 
1. Geometric Structures and Energy of OMpCA and Its One-Water Complexes<smiles>COC(=O)/C=C/c1ccc(O)cc1</smiles>

I. s-cis OH anti-OMpCA<smiles>COC(=O)/C=C/c1ccc(O)cc1</smiles>

III. s-trans $\mathrm{OH}$ anti-OMpCA<smiles>COC(=O)/C=C/c1ccc(O)cc1</smiles>

II. s-cis $\mathrm{OH}$ syn-OMpCA<smiles>COC(=O)/C=C/c1ccc(O)cc1</smiles>

IV. s-trans $\mathrm{OH}$ syn-OMpCA

Fig. S1: Schematic geometric structures of four $\mathrm{S}_{0}$ conformers of OMpCA in the trans form.

Table S1: Relative Energy (eV) of Four $\mathrm{S}_{0}$ Conformers of OMpCA in the Trans Form Calculated by the B3LYP Method with Different Basis Sets.

\begin{tabular}{|c|c|c|}
\hline Energy & $6-31 \mathrm{G}^{*}(\mathrm{OPT})$ & $6-31++\mathrm{G}^{* *}(\mathrm{SP})$ \\
\hline s-cis OH anti-OMpCA & $\mathbf{0 . 0}$ & $\mathbf{0 . 0}$ \\
\hline s-cis OH syn-OMpCA & 0.059 & 0.005 \\
\hline s-trans OH anti-OMpCA & 0.092 & 0.041 \\
\hline s-trans OH syn-OMpCA & 0.147 & 0.042 \\
\hline
\end{tabular}

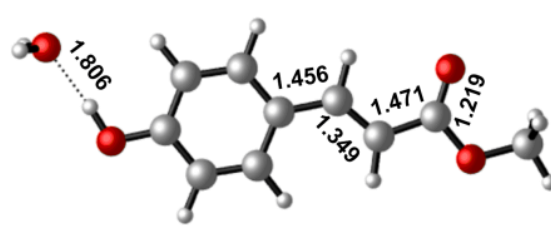

OH anti-OMpCA-W1-MIN

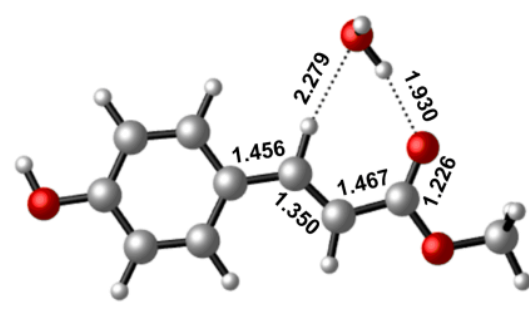

OH anti-OMpCA-W2-MIN

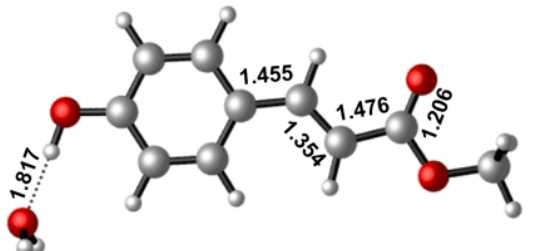

OH syn-OMpCA-W1-MIN

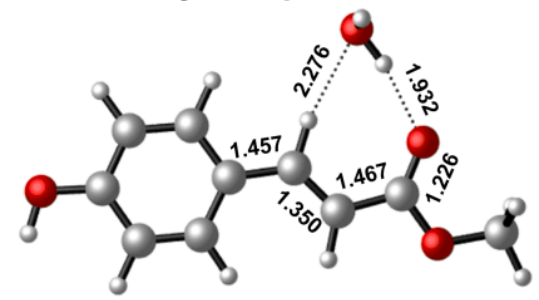

OH syn-OMpCA-W2-MIN

Fig. S2: Schematic geometric structures of four $\mathrm{S}_{0}$ conformers of $\mathrm{OMpCA}-\mathrm{H}_{2} \mathrm{O}$ complexes. 
Table S2: Relative Energy $(\mathrm{eV})$ of Four $\mathrm{S}_{0}$ Conformers of $\mathrm{OMpCA}-\mathrm{H}_{2} \mathrm{O}$ in the Trans Form Calculated by the B3LYP and MS-CASPT2//CASSCF Methods with Different Basis Sets.

\begin{tabular}{|c|c|c|c|}
\hline Energy & $6-31 \mathrm{G}^{*}(\mathrm{OPT})$ & $6-31++\mathrm{G}^{* *}(\mathrm{SP})$ & MS-CASPT2 \\
\hline OH anti-OMpCA-W1 & 0.0 & 0.0 & 0.0 \\
\hline OH syn-OMpCA-W1 & 0.01 & 0.05 & - \\
\hline OH anti-OMpCA-W2 & 0.01 & 0.04 & 0.03 \\
\hline OH syn-OMpCA-W2 & 0.02 & 0.05 & - \\
\hline
\end{tabular}

2. Active Orbitals used in CASSCF and MS-CASPT2 Computations CASSCF $(10,8)$ for ${ }^{1} \pi \pi^{*}$ state

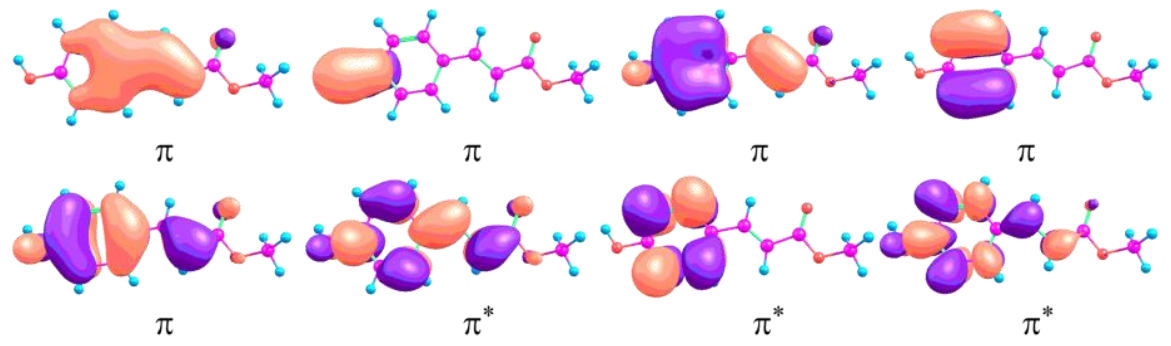

$\operatorname{CASSCF}(10,8)$ for ${ }^{1} \mathrm{n} \pi^{*}$ state
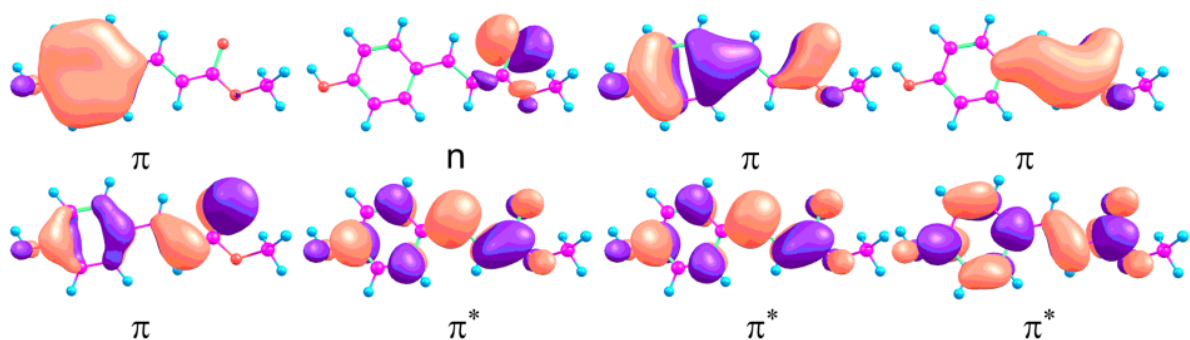

MS-CASPT2//CASSCF $(12,9)$ for ${ }^{1} \pi \pi^{*}$ and ${ }^{1} n \pi^{*}$ states

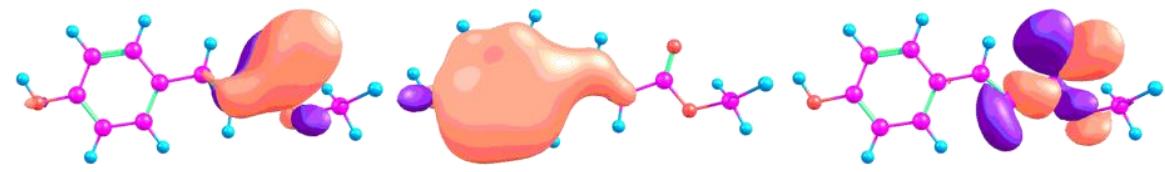

$\pi$

$\mathrm{n}$

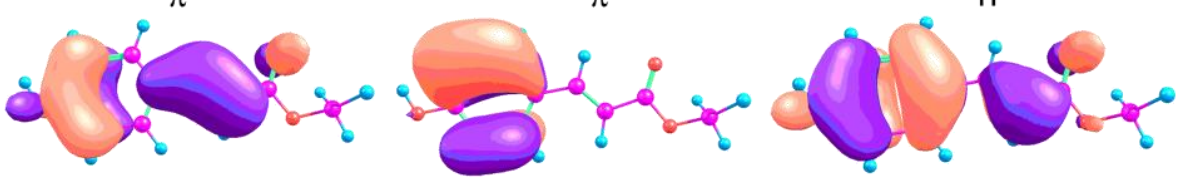

$\pi$

$\pi$

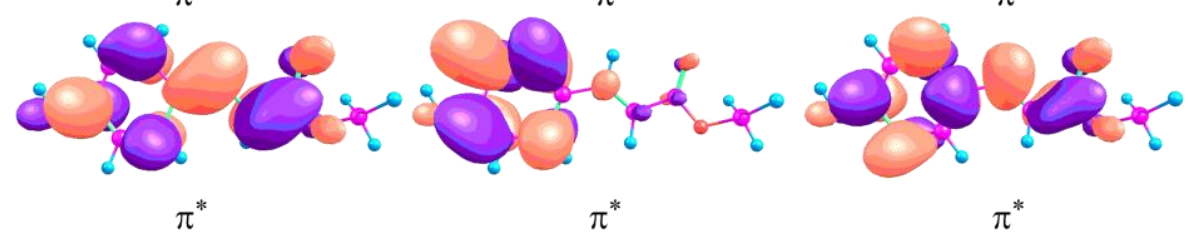

$\operatorname{CASSCF}(10,8)$ for ${ }^{1} \pi \pi^{*}$ state
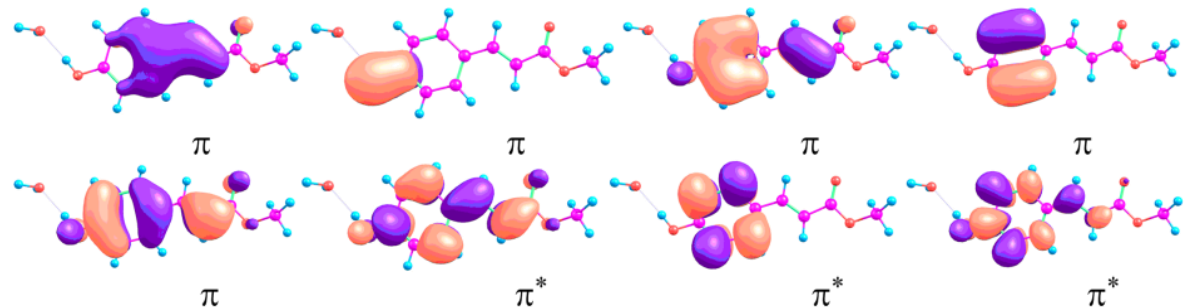
CASSCF $(10,8)$ for ${ }^{1} \mathrm{n} \pi^{*}$ state

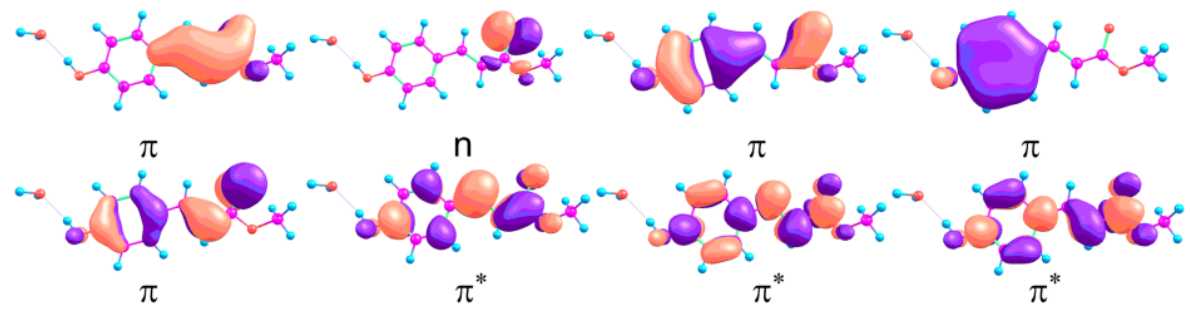

MS-CASPT2//CASSCF $(12,9)$ for ${ }^{1} \pi \pi^{*}$ and ${ }^{1} n \pi^{*}$ states
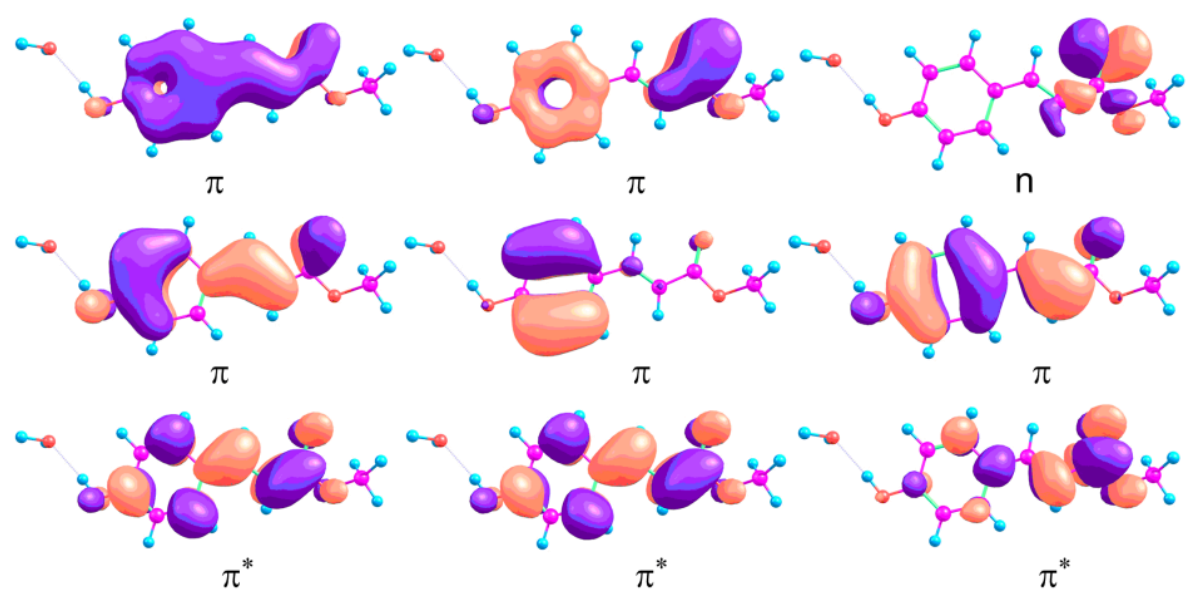

$\operatorname{CASSCF}(10,8)$ for ${ }^{1} \pi \pi^{*}$ state

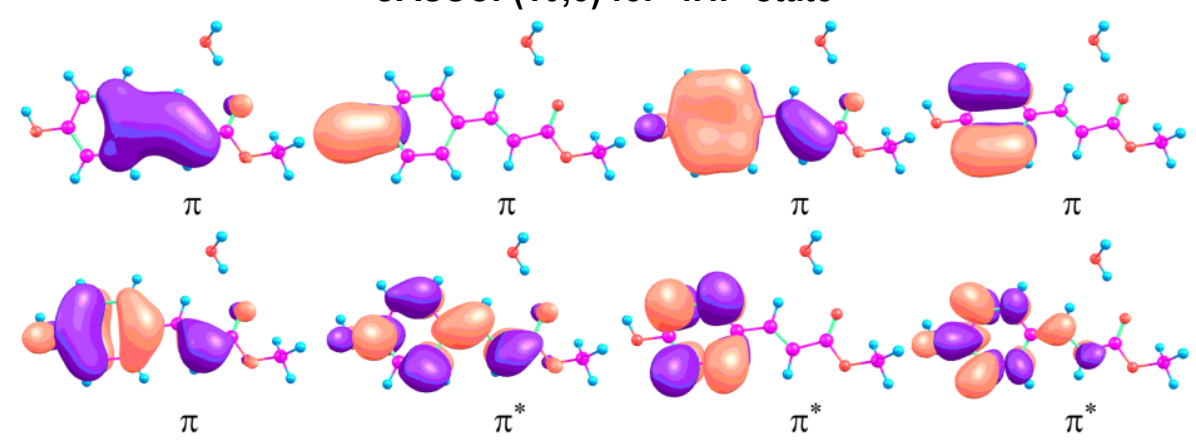

CASSCF $(10,8)$ for ${ }^{1} \mathrm{n} \pi^{*}$ state

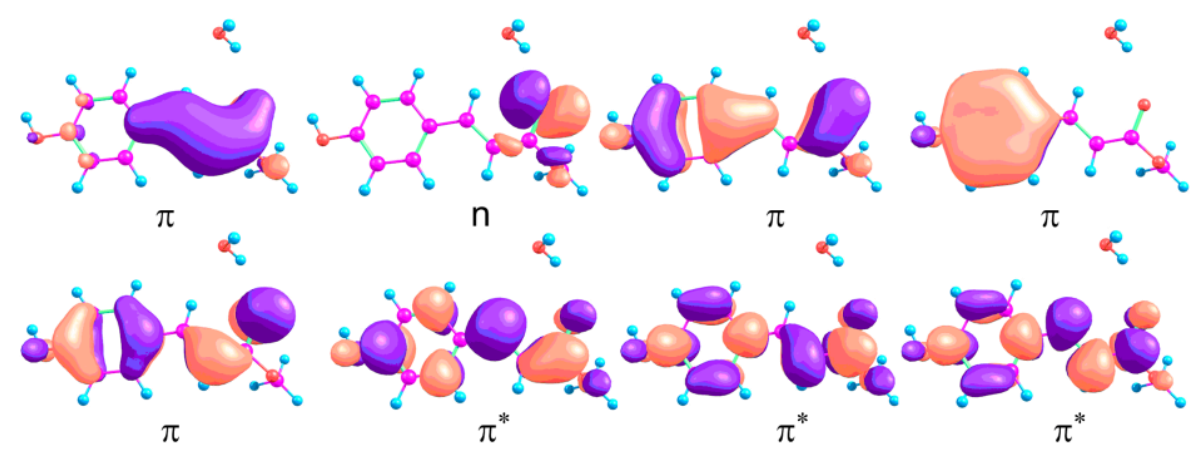


MS-CASPT2//CASSCF $(12,9)$ for ${ }^{1} \pi \pi^{*}$ and ${ }^{1} \mathrm{n} \pi^{*}$ states
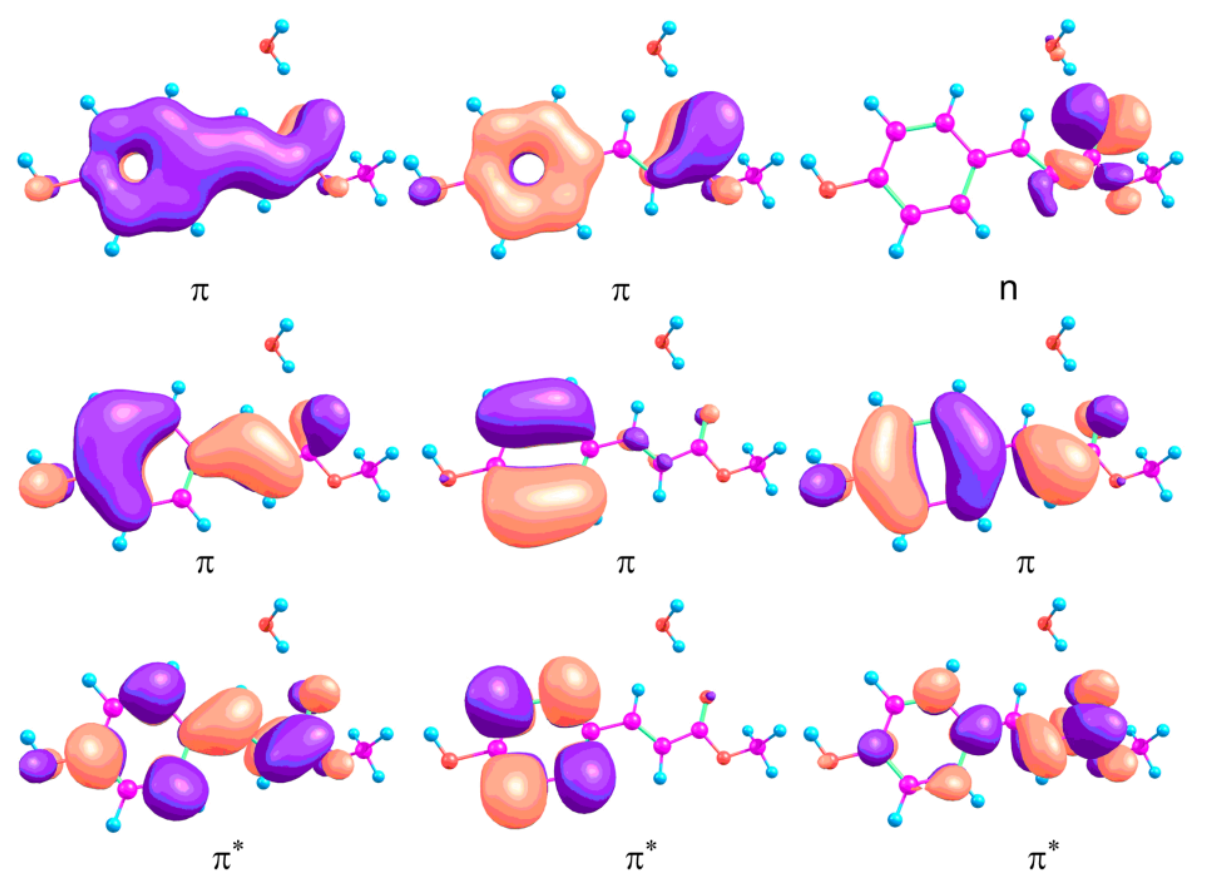

Fig. S3: Active spaces used in the CASSCF optimizations and MS- CASPT2 single-point energy computations.

3. Molecular Orbitals Involved in Vertical Electronic Transitions
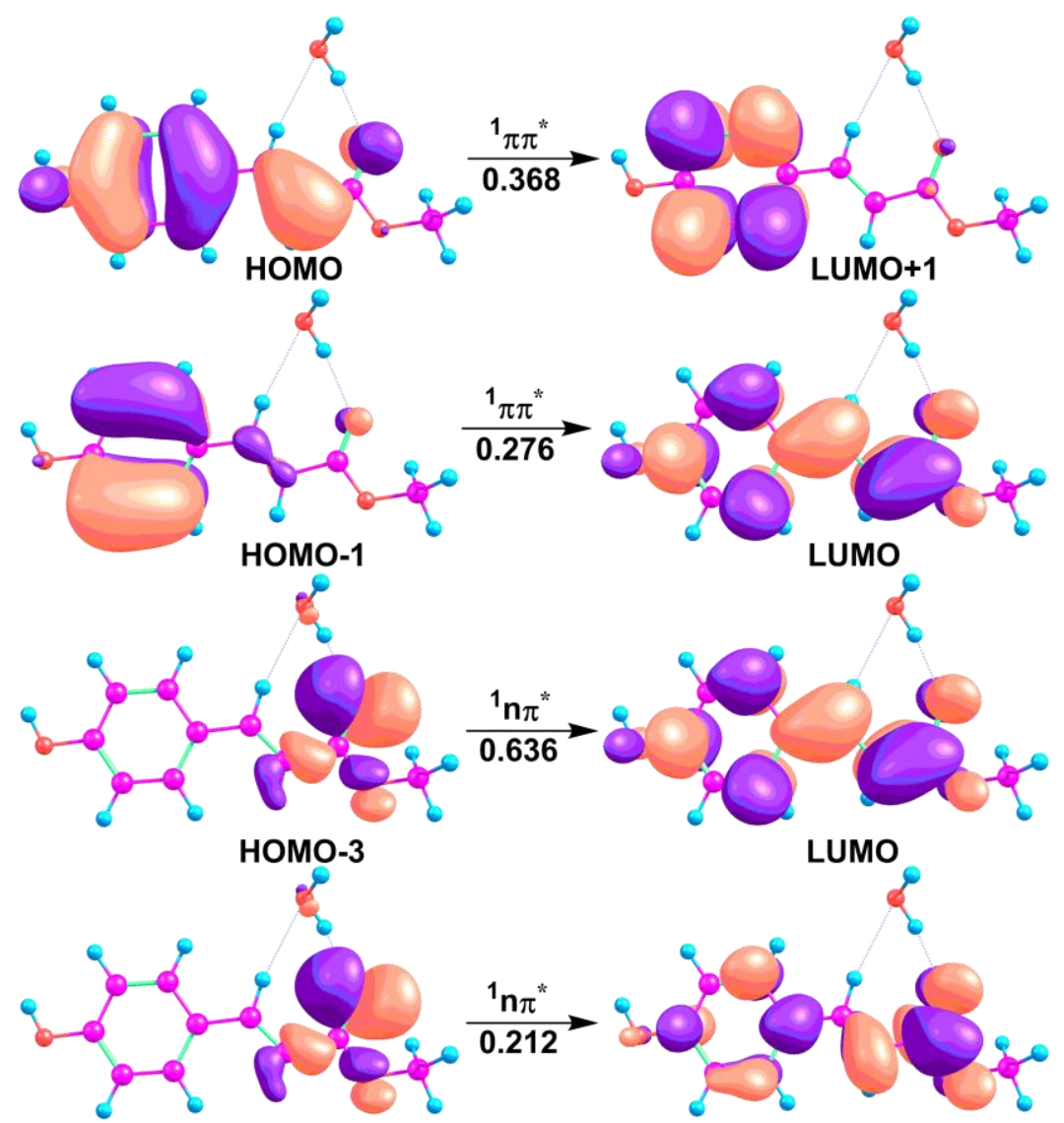

HOMO-3 


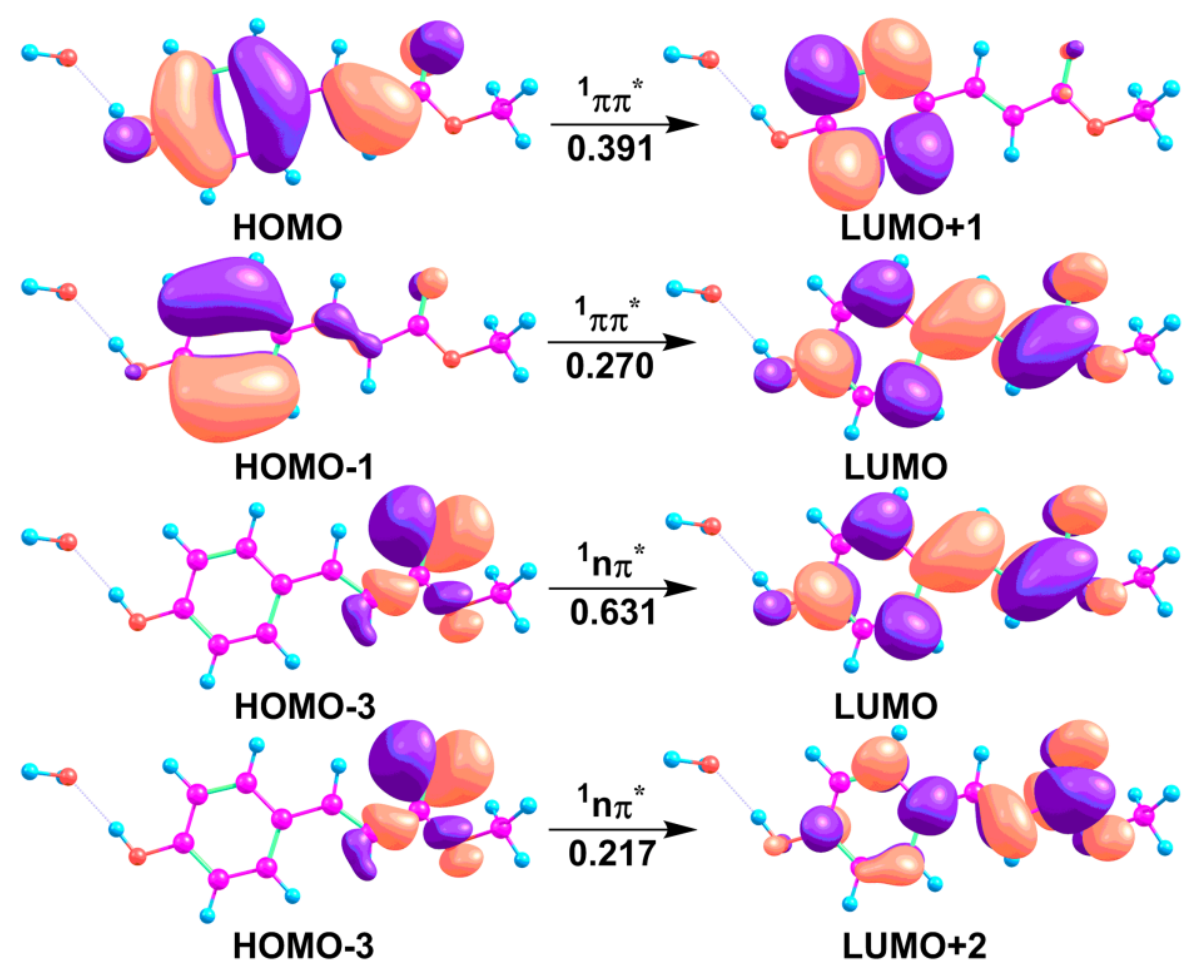

Fig. S4: MS-CASPT2 computed molecular orbitals involved in the $\mathrm{S}_{0} \rightarrow{ }^{1} \pi \pi^{*}$ and $\mathrm{S}_{0} \rightarrow{ }^{1} \mathrm{n} \pi^{*}$ electronic transitions

\section{4. $V^{\prime}\left({ }^{1} \pi \pi^{*}\right)$ and $V\left({ }^{1} \pi \pi^{*}\right)$ of OMpCA and lts Two One-Water Complexes}

Table S3: MS-CASPT2//CASSCF Computed Vertical Excitation Energies (Kcal/Mol) to the ${ }^{1} n \pi^{*}$, $V^{\prime}\left({ }^{1} \pi \pi^{*}\right)$, and $V\left({ }^{1} \pi \pi^{*}\right)$ Excited Singlet States at the Franck-Condon Points of OMpCA, and Its Two Water Complexes OMpCA-W1 and OMpCA-W2.

\begin{tabular}{|c|c|c|c|}
\hline & OMpCA & OMpCA-W1 & OMpCA-W2 \\
\hline \hline $\mathrm{V}^{\prime}\left(\pi \pi^{*}\right)$ & 99.6 & 90.2 & 90.2 \\
\hline $\mathrm{V}\left(\pi \pi^{*}\right)$ & 104.6 & 103.5 & 103.8 \\
\hline $\mathrm{n} \pi^{*}$ & 109.3 & 119.1 & 121.9 \\
\hline
\end{tabular}

\section{Cartesian Coordinates of All Optimized Structures and Paths}

In xyz format (unit: angström)

\section{CASSCF method}

OMpCA, OMpCA-W1 and OMpCA-W2

SO-MIN

$\begin{array}{lrrr}\text { C } & -0.006740770 & 4.084732840 & 0.102234010 \\ \text { C } & -0.006141880 & 2.686029960 & 0.065949620 \\ \text { C } & 1.191055690 & 1.956127620 & 0.055832570\end{array}$




\begin{tabular}{|c|c|c|c|}
\hline C & 2.406778680 & 2.676441460 & 0.083479550 \\
\hline C & 2.415689710 & 4.065374330 & 0.119582260 \\
\hline C & 1.205419640 & 4.772894230 & 0.129021270 \\
\hline $\mathrm{H}$ & -0.938722080 & 4.623682240 & 0.109348430 \\
\hline $\mathrm{H}$ & -0.946439590 & 2.164672820 & 0.045494560 \\
\hline $\mathrm{H}$ & 3.344663330 & 2.153900280 & 0.076834920 \\
\hline $\mathrm{H}$ & 3.337943740 & 4.614935070 & 0.140637590 \\
\hline 0 & 1.278944130 & 6.123714790 & 0.164607880 \\
\hline C & 1.121412010 & 0.490872530 & 0.017303370 \\
\hline C & 2.114405100 & -0.371067910 & 0.002238590 \\
\hline $\mathrm{H}$ & 0.127028510 & 0.076752260 & -0.000751000 \\
\hline $\mathrm{H}$ & 3.149949460 & -0.090114630 & 0.017139490 \\
\hline C & 1.830164890 & -1.829080090 & -0.037678680 \\
\hline 0 & 0.748461690 & -2.325622420 & -0.058508160 \\
\hline 0 & 2.952489940 & -2.531242700 & -0.047650440 \\
\hline C & 2.828442680 & -3.941362440 & -0.085148230 \\
\hline $\mathrm{H}$ & 2.306013290 & -4.252068840 & -0.978280150 \\
\hline $\mathrm{H}$ & 3.836371920 & -4.325819050 & -0.087718990 \\
\hline $\mathrm{H}$ & 2.293074480 & -4.297764120 & 0.782903820 \\
\hline $\mathrm{H}$ & 0.413266740 & 6.507300120 & 0.168192450 \\
\hline \multicolumn{4}{|c|}{ PP-MIN } \\
\hline C & -0.031904850 & 4.113075740 & 0.102777830 \\
\hline C & -0.051278820 & 2.679518340 & 0.065447350 \\
\hline C & 1.179826400 & 1.903134520 & 0.054381610 \\
\hline C & 2.428483360 & 2.637277990 & 0.082634040 \\
\hline C & 2.433514950 & 4.082110900 & 0.120151720 \\
\hline C & 1.210843810 & 4.796906830 & 0.129681650 \\
\hline $\mathrm{H}$ & -0.957355660 & 4.659250720 & 0.110120700 \\
\hline $\mathrm{H}$ & -0.992316880 & 2.164639050 & 0.045149050 \\
\hline $\mathrm{H}$ & 3.363721440 & 2.116515970 & 0.076028820 \\
\hline $\mathrm{H}$ & 3.357446480 & 4.625086840 & 0.141048390 \\
\hline 0 & 1.289400290 & 6.148064520 & 0.165308470 \\
\hline C & 1.101172780 & 0.496336180 & 0.017302280 \\
\hline C & 2.118720380 & -0.381649660 & 0.002005630 \\
\hline $\mathrm{H}$ & 0.111318270 & 0.072597150 & -0.000973750 \\
\hline $\mathrm{H}$ & 3.150813940 & -0.092454490 & 0.017089080 \\
\hline C & 1.841767280 & -1.829354100 & -0.037586670 \\
\hline 0 & 0.759154860 & -2.330763380 & -0.058527830 \\
\hline 0 & 2.966004960 & -2.534821480 & -0.047656540 \\
\hline C & 2.837809540 & -3.943119680 & -0.085138170 \\
\hline $\mathrm{H}$ & 2.314525820 & -4.253882810 & -0.977985350 \\
\hline $\mathrm{H}$ & 3.844615890 & -4.331212360 & -0.087820990 \\
\hline $\mathrm{H}$ & 2.301610670 & -4.299565090 & 0.782625090 \\
\hline $\mathrm{H}$ & 0.425636420 & 6.535596640 & 0.169002310 \\
\hline
\end{tabular}




\begin{tabular}{|c|c|c|c|}
\hline \multicolumn{4}{|c|}{ NP-MIN } \\
\hline C & 0.064750490 & 4.144634800 & 0.104216720 \\
\hline C & 0.024941670 & 2.772892620 & 0.068250100 \\
\hline C & 1.197113560 & 1.989559090 & 0.056660170 \\
\hline C & 2.422420300 & 2.696013270 & 0.084211590 \\
\hline C & 2.467224690 & 4.063116150 & 0.120097200 \\
\hline C & 1.287266200 & 4.808705140 & 0.130645350 \\
\hline $\mathrm{H}$ & -0.854140510 & 4.707755080 & 0.111973950 \\
\hline $\mathrm{H}$ & -0.932193520 & 2.281314530 & 0.048298780 \\
\hline $\mathrm{H}$ & 3.351975220 & 2.157726810 & 0.077177920 \\
\hline $\mathrm{H}$ & 3.406846200 & 4.584225640 & 0.140688370 \\
\hline 0 & 1.393157760 & 6.160425960 & 0.166586420 \\
\hline C & 1.091776010 & 0.551841560 & 0.018502390 \\
\hline C & 2.188669500 & -0.369800450 & 0.002627010 \\
\hline $\mathrm{H}$ & 0.095365850 & 0.148807590 & 0.000679440 \\
\hline $\mathrm{H}$ & 3.202014760 & -0.018933340 & 0.019093260 \\
\hline C & 2.068389750 & -1.716607640 & -0.033098930 \\
\hline 0 & 0.847686980 & -2.359523460 & -0.058230730 \\
\hline 0 & 3.116953380 & -2.554320720 & -0.047495490 \\
\hline C & 2.909497250 & -3.949100800 & -0.084686790 \\
\hline $\mathrm{H}$ & 2.379704690 & -4.245659190 & -0.981740830 \\
\hline $\mathrm{H}$ & 3.893649260 & -4.389828700 & -0.089538570 \\
\hline $\mathrm{H}$ & 2.368040370 & -4.291135140 & 0.788907670 \\
\hline $\mathrm{H}$ & 0.535121310 & 6.560155860 & 0.170561120 \\
\hline \multicolumn{4}{|c|}{ S0-W1-MIN } \\
\hline C & -2.713243490 & 0.773820500 & -0.001167410 \\
\hline C & -1.342510240 & 1.038728060 & 0.015252490 \\
\hline C & -0.394784010 & 0.008413980 & -0.005394440 \\
\hline C & -0.867453880 & -1.319289040 & -0.043697910 \\
\hline C & -2.226931980 & -1.594933750 & -0.060340150 \\
\hline C & -3.159753110 & -0.547774150 & -0.039111920 \\
\hline $\mathrm{H}$ & -3.423348470 & 1.581003090 & 0.015249100 \\
\hline $\mathrm{H}$ & -1.009756420 & 2.061300730 & 0.044515800 \\
\hline $\mathrm{H}$ & -0.173225220 & -2.138506740 & -0.060541640 \\
\hline $\mathrm{H}$ & -2.586532780 & -2.606629710 & -0.089529850 \\
\hline 0 & -4.464857850 & -0.877745960 & -0.056785880 \\
\hline C & 1.019825540 & 0.358074660 & 0.013613730 \\
\hline C & 2.067895120 & -0.453790190 & -0.000471760 \\
\hline $\mathrm{H}$ & 1.235448310 & 1.412939100 & 0.042870510 \\
\hline $\mathrm{H}$ & 1.992017930 & -1.523485740 & -0.029147330 \\
\hline C & 3.438388980 & 0.107193740 & 0.023827040 \\
\hline 0 & 3.717697600 & 1.265067830 & 0.056213420 \\
\hline 0 & 4.349277380 & -0.856224840 & 0.004851530 \\
\hline $\mathrm{C}$ & 5.706277040 & -0.456103460 & 0.024845560 \\
\hline
\end{tabular}




\begin{tabular}{|c|c|c|c|}
\hline $\mathrm{H}$ & 5.923779610 & 0.105170670 & 0.922128480 \\
\hline $\mathrm{H}$ & 6.283121600 & -1.367725460 & 0.004916690 \\
\hline $\mathrm{H}$ & 5.936078870 & 0.151445720 & -0.838593770 \\
\hline $\mathrm{H}$ & -5.019905510 & -0.102987870 & -0.040073090 \\
\hline 0 & -6.271910970 & 1.389472980 & -0.008873870 \\
\hline $\mathrm{H}$ & -6.839896870 & 1.450479560 & 0.748089220 \\
\hline $\mathrm{H}$ & -6.829910750 & 1.490670700 & -0.768951410 \\
\hline \multicolumn{4}{|c|}{ PP-W1-MIN } \\
\hline C & -2.747655500 & 0.794856310 & -0.000610300 \\
\hline C & -1.344309640 & 1.089556510 & 0.016855620 \\
\hline C & -0.342089910 & 0.035243710 & -0.004105810 \\
\hline C & -0.822770010 & -1.330089290 & -0.043514000 \\
\hline C & -2.239674120 & -1.613231300 & -0.060772710 \\
\hline C & -3.182367900 & -0.556007370 & -0.039320160 \\
\hline $\mathrm{H}$ & -3.464663400 & 1.593355610 & 0.015553530 \\
\hline $\mathrm{H}$ & -1.020129870 & 2.112214450 & 0.046126470 \\
\hline $\mathrm{H}$ & -0.130597450 & -2.146787760 & -0.060343830 \\
\hline $\mathrm{H}$ & -2.591057220 & -2.625731610 & -0.089969660 \\
\hline 0 & -4.487754160 & -0.891866900 & -0.057205460 \\
\hline C & 1.019690600 & 0.382562210 & 0.014460430 \\
\hline C & 2.081189680 & -0.449190420 & -0.000206550 \\
\hline $\mathrm{H}$ & 1.245568890 & 1.435051620 & 0.043833910 \\
\hline $\mathrm{H}$ & 1.994619870 & -1.517140180 & -0.029027470 \\
\hline C & 3.446553050 & 0.098583500 & 0.023677060 \\
\hline 0 & 3.735029210 & 1.256832430 & 0.056272020 \\
\hline 0 & 4.356415480 & -0.870080020 & 0.004346990 \\
\hline C & 5.712910000 & -0.473061830 & 0.024181240 \\
\hline $\mathrm{H}$ & 5.933183840 & 0.087749030 & 0.921292800 \\
\hline $\mathrm{H}$ & 6.288444830 & -1.385792560 & 0.004021640 \\
\hline $\mathrm{H}$ & 5.945305290 & 0.134271110 & -0.838939590 \\
\hline $\mathrm{H}$ & -5.045036380 & -0.118996950 & -0.040625000 \\
\hline 0 & -6.289339450 & 1.383073400 & -0.009548570 \\
\hline $\mathrm{H}$ & -6.858080730 & 1.441586700 & 0.747082550 \\
\hline $\mathrm{H}$ & -6.847598610 & 1.481623990 & -0.769822010 \\
\hline \multicolumn{4}{|c|}{ NP-W1-MIN } \\
\hline C & -2.746895980 & 0.723705010 & -0.003050270 \\
\hline C & -1.405614060 & 1.015467440 & 0.013813130 \\
\hline C & -0.415635070 & 0.010757140 & -0.005760350 \\
\hline C & -0.883365500 & -1.323677070 & -0.043995370 \\
\hline C & -2.219515680 & -1.620013470 & -0.060956710 \\
\hline C & -3.175772110 & -0.601887860 & -0.040790240 \\
\hline $\mathrm{H}$ & -3.473393160 & 1.517655290 & 0.012815350 \\
\hline $\mathrm{H}$ & -1.099743020 & 2.047296280 & 0.043000430 \\
\hline $\mathrm{H}$ & -0.182187960 & -2.137757060 & -0.060529490 \\
\hline
\end{tabular}




\begin{tabular}{|c|c|c|c|}
\hline $\mathrm{H}$ & -2.556457930 & -2.640374880 & -0.090055000 \\
\hline 0 & -4.478491330 & -0.953925140 & -0.058965580 \\
\hline C & 0.977066650 & 0.382362600 & 0.013592370 \\
\hline C & 2.087505960 & -0.521872160 & -0.002422240 \\
\hline $\mathrm{H}$ & 1.187220010 & 1.436313810 & 0.042702960 \\
\hline $\mathrm{H}$ & 1.932158070 & -1.582711660 & -0.031402470 \\
\hline C & 3.387865800 & -0.151577250 & 0.016400150 \\
\hline 0 & 3.790906350 & 1.168600920 & 0.053993840 \\
\hline 0 & 4.409760840 & -1.023389300 & 0.000482050 \\
\hline C & 5.739724660 & -0.556217120 & 0.022885800 \\
\hline $\mathrm{H}$ & 5.946158870 & 0.006543260 & 0.925273060 \\
\hline $\mathrm{H}$ & 6.359190900 & -1.438811620 & 0.003982920 \\
\hline $\mathrm{H}$ & 5.959380890 & 0.053723250 & -0.845066980 \\
\hline $\mathrm{H}$ & -5.041793160 & -0.185994110 & -0.042444330 \\
\hline O & -6.353820920 & 1.273690780 & -0.012061640 \\
\hline $\mathrm{H}$ & -6.926642590 & 1.281337900 & 0.743569220 \\
\hline $\mathrm{H}$ & -6.917603090 & 1.321362350 & -0.773006390 \\
\hline \multicolumn{4}{|c|}{ SO-W2-MIN } \\
\hline C & -2.870488780 & 0.825116280 & -0.021488280 \\
\hline C & -1.480748940 & 0.977613600 & -0.018647010 \\
\hline C & -0.628106540 & -0.132298910 & 0.010561100 \\
\hline C & -1.208002200 & -1.416240470 & 0.039551100 \\
\hline C & -2.588235420 & -1.577392260 & 0.038310300 \\
\hline C & -3.422574760 & -0.453171400 & 0.007055860 \\
\hline $\mathrm{H}$ & -3.507209260 & 1.692894070 & -0.046227050 \\
\hline $\mathrm{H}$ & -1.058751210 & 1.966231610 & -0.043091140 \\
\hline $\mathrm{H}$ & -0.585463380 & -2.290655930 & 0.064787710 \\
\hline $\mathrm{H}$ & -3.033473670 & -2.554258640 & 0.061073700 \\
\hline 0 & -4.758367590 & -0.673546340 & 0.007249540 \\
\hline C & 0.806143840 & 0.105036260 & 0.008123380 \\
\hline C & 1.788288060 & -0.791741580 & 0.000986620 \\
\hline $\mathrm{H}$ & 1.090991540 & 1.142094100 & 0.011273030 \\
\hline $\mathrm{H}$ & 1.626834130 & -1.852023850 & -0.005574070 \\
\hline C & 3.199442300 & -0.351047570 & -0.004349730 \\
\hline 0 & 3.585501610 & 0.781978160 & -0.000615940 \\
\hline 0 & 4.024124930 & -1.381446230 & -0.013330700 \\
\hline C & 5.413810510 & -1.104008270 & -0.025908830 \\
\hline $\mathrm{H}$ & 5.696678630 & -0.548797740 & 0.856457220 \\
\hline $\mathrm{H}$ & 5.904056230 & -2.064538640 & -0.037438450 \\
\hline $\mathrm{H}$ & 5.678190590 & -0.536580710 & -0.906115920 \\
\hline 0 & 2.137539100 & 3.386318870 & -0.014788170 \\
\hline $\mathrm{H}$ & 2.734794330 & 2.655453490 & -0.140558500 \\
\hline & 2.343174900 & 3.725162510 & 0.845574290 \\
\hline & -5.232950930 & 0.145304590 & -0.020314070 \\
\hline
\end{tabular}




\begin{tabular}{|c|c|c|c|}
\hline \multicolumn{4}{|c|}{ PP-W2-MIN } \\
\hline C & -2.910785980 & 0.847882290 & -0.016567930 \\
\hline C & -1.485129580 & 1.024347060 & -0.013460080 \\
\hline C & -0.584659540 & -0.122370520 & 0.010414610 \\
\hline C & -1.175438160 & -1.447326990 & 0.033932600 \\
\hline C & -2.615049990 & -1.605668390 & 0.033484780 \\
\hline C & -3.456200520 & -0.463446940 & 0.007176560 \\
\hline $\mathrm{H}$ & -3.554633880 & 1.707900180 & -0.038767370 \\
\hline $\mathrm{H}$ & -1.067398940 & 2.012194770 & -0.039383570 \\
\hline $\mathrm{H}$ & -0.555367080 & -2.319711450 & 0.056807560 \\
\hline $\mathrm{H}$ & -3.057995620 & -2.581506310 & 0.051599410 \\
\hline 0 & -4.792745510 & -0.687382230 & 0.007038410 \\
\hline C & 0.814583200 & 0.118278510 & 0.006829470 \\
\hline C & 1.797285470 & -0.785564390 & 0.006443310 \\
\hline $\mathrm{H}$ & 1.103535430 & 1.154341980 & 0.003216770 \\
\hline $\mathrm{H}$ & 1.630510120 & -1.844590940 & 0.007679910 \\
\hline C & 3.205507280 & -0.351076420 & -0.002863660 \\
\hline 0 & 3.596628360 & 0.782043400 & -0.006093830 \\
\hline 0 & 4.029945950 & -1.383917730 & -0.006930580 \\
\hline C & 5.418968750 & -1.107570350 & -0.023271080 \\
\hline $\mathrm{H}$ & 5.704398080 & -0.547175330 & 0.855122580 \\
\hline $\mathrm{H}$ & 5.908993060 & -2.068421500 & -0.029767160 \\
\hline $\mathrm{H}$ & 5.683206450 & -0.545606720 & -0.907164510 \\
\hline 0 & 2.161061560 & 3.386705980 & -0.018034950 \\
\hline $\mathrm{H}$ & 2.750759500 & 2.649836450 & -0.146408190 \\
\hline $\mathrm{H}$ & 2.374515820 & 3.723124720 & 0.841370640 \\
\hline $\mathrm{H}$ & -5.269296210 & 0.130135920 & -0.019847730 \\
\hline \multicolumn{4}{|c|}{ NP-W2-MIN } \\
\hline C & -2.851624980 & 0.695020200 & -0.238646750 \\
\hline C & -1.508821000 & 0.953420190 & -0.115681170 \\
\hline C & -0.565326480 & -0.076135160 & 0.073861230 \\
\hline C & -1.074817420 & -1.392956490 & 0.131889440 \\
\hline C & -2.413062460 & -1.655465330 & 0.009918490 \\
\hline C & -3.321280640 & -0.613427610 & -0.177636860 \\
\hline $\mathrm{H}$ & -3.543085120 & 1.508899730 & -0.383148190 \\
\hline $\mathrm{H}$ & -1.165277380 & 1.971775110 & -0.166468710 \\
\hline $\mathrm{H}$ & -0.406703300 & -2.221863980 & 0.276774670 \\
\hline $\mathrm{H}$ & -2.785226740 & -2.662492360 & 0.056778140 \\
\hline 0 & -4.633825130 & -0.932010950 & -0.292511550 \\
\hline C & 0.831887320 & 0.263821450 & 0.195193230 \\
\hline C & 1.905608820 & -0.663817750 & 0.373517220 \\
\hline $\mathrm{H}$ & 1.073623260 & 1.309306130 & 0.141856670 \\
\hline $\mathrm{H}$ & 1.717338710 & -1.718013340 & 0.442518650 \\
\hline C & 3.213730990 & -0.322182630 & 0.485973020 \\
\hline
\end{tabular}




$\begin{array}{lrrr}\text { O } & 3.659425110 & 0.983334380 & 0.428012400 \\ \text { O } & 4.232633740 & -1.165974560 & 0.692790470 \\ \mathrm{C} & 5.018870270 & -1.485328390 & -0.442100410 \\ \mathrm{H} & 5.523577920 & -0.607712460 & -0.826755180 \\ \mathrm{H} & 5.750914800 & -2.205152080 & -0.110269050 \\ \mathrm{H} & 4.401230060 & -1.919777060 & -1.218689450 \\ \mathrm{O} & 2.162794170 & 3.727458190 & 0.376474990 \\ \mathrm{H} & 2.963121840 & 3.343854840 & 0.043403150 \\ \mathrm{H} & 2.236041370 & 3.667976260 & 1.319556180 \\ \mathrm{H} & -5.155262580 & -0.151496400 & -0.415337780\end{array}$

\section{Minimum-energy isomerization paths for the ${ }^{1} \pi \pi^{*}$ excited-state \\ OMpCA}

C2-C3-C4-C5 angle: 180

\begin{tabular}{|c|c|c|c|}
\hline C & -0.035094710 & 4.113650000 & 0.102772140 \\
\hline C & -0.052372120 & 2.686833620 & 0.065632090 \\
\hline C & 1.178251740 & 1.925858490 & 0.054958090 \\
\hline C & 2.429139890 & 2.643655050 & 0.082796560 \\
\hline C & 2.440009420 & 4.080397590 & 0.120152640 \\
\hline C & 1.213613900 & 4.794625630 & 0.129643100 \\
\hline $\mathrm{H}$ & -0.957993250 & 4.664020080 & 0.110243350 \\
\hline $\mathrm{H}$ & -0.990688580 & 2.166647500 & 0.045219750 \\
\hline $\mathrm{H}$ & 3.359471290 & 2.113884130 & 0.075910520 \\
\hline $\mathrm{H}$ & 3.362413550 & 4.625835650 & 0.141103920 \\
\hline 0 & 1.292046070 & 6.145981330 & 0.165274940 \\
\hline C & 1.096318550 & 0.484027620 & 0.016947710 \\
\hline C & 2.111236050 & -0.383008010 & 0.001909810 \\
\hline $\mathrm{H}$ & 0.104976880 & 0.065211470 & -0.001204460 \\
\hline $\mathrm{H}$ & 3.142315850 & -0.089760860 & 0.017092750 \\
\hline C & 1.839138660 & -1.834160380 & -0.037739700 \\
\hline 0 & 0.757368640 & -2.336572440 & -0.058712590 \\
\hline 0 & 2.965142940 & -2.535131940 & -0.047665500 \\
\hline C & 2.842230160 & -3.944319810 & -0.085129790 \\
\hline $\mathrm{H}$ & 2.320190620 & -4.256623580 & -0.978121350 \\
\hline $\mathrm{H}$ & 3.850477760 & -4.328418140 & -0.087681980 \\
\hline $\mathrm{H}$ & 2.307243410 & -4.302307970 & 0.782698600 \\
\hline $\mathrm{H}$ & 0.428094620 & 6.532963320 & 0.168964120 \\
\hline \multicolumn{4}{|c|}{ C2-C3-C4-C5 angle: 170} \\
\hline C & -0.041757950 & 4.097676370 & 0.318047470 \\
\hline C & -0.055735470 & 2.678746460 & 0.161333980 \\
\hline C & 1.176238870 & 1.920622470 & 0.125061840 \\
\hline C & 2.424501690 & 2.633403000 & 0.244243310 \\
\hline C & 2.432001710 & 4.062142370 & 0.399225930 \\
\hline C & 1.204600620 & 4.773146970 & 0.4344330 \\
\hline
\end{tabular}




$\begin{array}{lrrr}\text { H } & -0.965214760 & 4.646514460 & 0.346169780 \\ \text { H } & -0.991985610 & 2.162335690 & 0.071547740 \\ \text { H } & 3.355377370 & 2.104979650 & 0.222586430 \\ \text { H } & 3.352527010 & 4.603608430 & 0.490003280 \\ \text { O } & 1.278938460 & 6.115167730 & 0.582309890 \\ \mathrm{C} & 1.098643410 & 0.487497460 & -0.031705910 \\ \mathrm{C} & 2.112934680 & -0.381282920 & -0.045279590 \\ \mathrm{H} & 0.107656470 & 0.069244620 & -0.072728190 \\ \mathrm{H} & 3.143390830 & -0.088072340 & -0.004052270 \\ \mathrm{C} & 1.840614960 & -1.831892330 & 0.005346310 \\ \mathrm{O} & 0.759586810 & -2.336192950 & -0.011265220 \\ \mathrm{O} & 2.965738930 & -2.529738240 & 0.086265760 \\ \mathrm{C} & 2.842619230 & -3.937524030 & 0.159009940 \\ \mathrm{H} & 2.347722280 & -4.321679870 & -0.721300620 \\ \mathrm{H} & 3.850111110 & -4.319035870 & 0.218236120 \\ \mathrm{H} & 2.281139990 & -4.225111940 & 1.036228810 \\ \mathrm{H} & 0.414475950 & 6.501189710 & 0.594849010\end{array}$

C2-C3-C4-C5 angle: 160

$\begin{array}{lrrr}\text { C } & -0.059080270 & 4.059751140 & 0.530558870 \\ \text { C } & -0.061923900 & 2.658863490 & 0.255553300 \\ \text { C } & 1.174218550 & 1.908917580 & 0.194081930 \\ \text { C } & 2.415389920 & 2.613573450 & 0.403272520 \\ \text { C } & 2.411436250 & 4.024925410 & 0.674517090 \\ \text { C } & 1.180050870 & 4.726923100 & 0.735672890 \\ \text { H } & -0.985737780 & 4.601712770 & 0.579052250 \\ \text { H } & -0.992763180 & 2.149244480 & 0.097293800 \\ \text { H } & 3.348795760 & 2.090522310 & 0.366477520 \\ \text { H } & 3.326479350 & 4.559837000 & 0.833736700 \\ \text { O } & 1.243561380 & 6.052442190 & 0.995411460 \\ \text { C } & 1.107821670 & 0.493693280 & -0.078744020 \\ \text { C } & 2.119385590 & -0.380166060 & -0.091129760 \\ \text { H } & 0.117148540 & 0.076302260 & -0.142443110 \\ \text { H } & 3.148654330 & -0.086123780 & -0.025052260 \\ \text { C } & 1.846217240 & -1.824329050 & 0.049995040 \\ \text { O } & 0.766764600 & -2.332195650 & 0.040498180 \\ \text { O } & 2.969366940 & -2.509797420 & 0.218247820 \\ \text { C } & 2.844910010 & -3.907410360 & 0.401359050 \\ \text { H } & 2.375521170 & -4.363014770 & -0.458586450 \\ \text { H } & 3.850552350 & -4.279912790 & 0.520023460 \\ \text { H } & 2.258814990 & -4.124487240 & 1.282799830 \\ \text { H } & 0.377082730 & 6.433452100 & 1.017261020 \\ \text { C2-C3-C4-C5 angle: } 150 & & \\ \text { C } & -0.086497590 & 4.001898490 & 0.737244130 \\ \text { C } & -0.072114920 & 2.628361780 & 0.348107320\end{array}$




\begin{tabular}{|c|c|c|c|}
\hline C & 1.170424580 & 1.890823960 & 0.263561080 \\
\hline C & 2.400488940 & 2.583582290 & 0.560946670 \\
\hline C & 2.378775700 & 3.968508820 & 0.944731290 \\
\hline C & 1.141318110 & 4.656588530 & 1.029568620 \\
\hline $\mathrm{H}$ & -1.018237100 & 4.533051620 & 0.804556210 \\
\hline $\mathrm{H}$ & -0.994624030 & 2.128991140 & 0.122848640 \\
\hline $\mathrm{H}$ & 3.337784300 & 2.068586460 & 0.510878340 \\
\hline $\mathrm{H}$ & 3.285348560 & 4.493127400 & 1.172106820 \\
\hline 0 & 1.188084660 & 5.956992170 & 1.397479690 \\
\hline C & 1.121517830 & 0.502851600 & -0.121023680 \\
\hline C & 2.128544670 & -0.379201420 & -0.132539840 \\
\hline $\mathrm{H}$ & 0.131708020 & 0.086725630 & -0.208255400 \\
\hline $\mathrm{H}$ & 3.156229370 & -0.083814490 & -0.044186830 \\
\hline C & 1.854993820 & -1.811154430 & 0.098605690 \\
\hline 0 & 0.777929440 & -2.324415060 & 0.102114860 \\
\hline 0 & 2.976211820 & -2.475691480 & 0.345984620 \\
\hline C & 2.851057830 & -3.854815230 & 0.637090400 \\
\hline $\mathrm{H}$ & 2.403944380 & -4.379689720 & -0.194738410 \\
\hline $\mathrm{H}$ & 3.854550100 & -4.211890500 & 0.809180270 \\
\hline $\mathrm{H}$ & 2.244346950 & -4.003746300 & 1.518704790 \\
\hline $\mathrm{H}$ & 0.318520170 & 6.330402160 & 1.427116490 \\
\hline \multicolumn{4}{|c|}{ C2-C3-C4-C5 angle: 140} \\
\hline C & -0.123316040 & 3.930962860 & 0.928180740 \\
\hline C & -0.087639160 & 2.591572040 & 0.434986590 \\
\hline C & 1.161561990 & 1.866029750 & 0.338638020 \\
\hline C & 2.376436880 & 2.539963990 & 0.728892220 \\
\hline C & 2.332460010 & 3.891389080 & 1.216690490 \\
\hline C & 1.088991850 & 4.566255660 & 1.311166240 \\
\hline $\mathrm{H}$ & -1.060391440 & 4.451480540 & 1.004168640 \\
\hline $\mathrm{H}$ & -0.998874090 & 2.107830850 & 0.140618810 \\
\hline $\mathrm{H}$ & 3.317263750 & 2.031731860 & 0.676019260 \\
\hline $\mathrm{H}$ & 3.227623170 & 4.400414810 & 1.514010540 \\
\hline 0 & 1.115025590 & 5.835244740 & 1.777852290 \\
\hline C & 1.134963260 & 0.513742880 & -0.150507770 \\
\hline C & 2.136181900 & -0.379417670 & -0.165173320 \\
\hline $\mathrm{H}$ & 0.145728660 & 0.099257540 & -0.259036220 \\
\hline $\mathrm{H}$ & 3.162516770 & -0.081174900 & -0.063327480 \\
\hline C & 1.865692650 & -1.793576680 & 0.157053290 \\
\hline 0 & 0.791421150 & -2.311890790 & 0.190869550 \\
\hline 0 & 2.988692920 & -2.431533180 & 0.461051690 \\
\hline C & 2.867327890 & -3.785065440 & 0.855441650 \\
\hline $\mathrm{H}$ & 2.424891690 & -4.373216480 & 0.064512500 \\
\hline $\mathrm{H}$ & 3.871549070 & -4.124139480 & 1.057274960 \\
\hline $\mathrm{H}$ & 2.258242120 & -3.869092220 & 1.744001950 \\
\hline
\end{tabular}




\begin{tabular}{|c|c|c|c|}
\hline $\mathrm{H}$ & 0.242459930 & 6.201587180 & 1.807466760 \\
\hline \multicolumn{4}{|c|}{ C2-C3-C4-C5 angle: 130} \\
\hline C & -0.160216910 & 3.897977450 & 1.054619140 \\
\hline C & -0.132898550 & 2.583051320 & 0.493093680 \\
\hline C & 1.098232360 & 1.798419390 & 0.441855410 \\
\hline C & 2.294941770 & 2.437901690 & 0.979900460 \\
\hline C & 2.237992490 & 3.774622790 & 1.520223780 \\
\hline C & 1.024076750 & 4.476787160 & 1.553822190 \\
\hline $\mathrm{H}$ & -1.088779530 & 4.438606130 & 1.090065370 \\
\hline $\mathrm{H}$ & -1.035472010 & 2.150010800 & 0.108345570 \\
\hline $\mathrm{H}$ & 3.224463950 & 1.907954540 & 0.999806980 \\
\hline $\mathrm{H}$ & 3.122500620 & 4.239590880 & 1.909172530 \\
\hline 0 & 1.045910880 & 5.723886550 & 2.084308820 \\
\hline C & 1.073266100 & 0.556437820 & -0.098539260 \\
\hline C & 2.141697620 & -0.383428310 & -0.099085750 \\
\hline $\mathrm{H}$ & 0.108048540 & 0.157659400 & -0.360079110 \\
\hline $\mathrm{H}$ & 3.165263610 & -0.063303580 & -0.096900030 \\
\hline C & 1.904445920 & -1.762716460 & 0.343004270 \\
\hline 0 & 0.831853650 & -2.254194520 & 0.523444780 \\
\hline 0 & 3.046117470 & -2.414216560 & 0.534761670 \\
\hline C & 2.948572590 & -3.753631730 & 0.980246330 \\
\hline $\mathrm{H}$ & 2.408066490 & -4.354623300 & 0.263204110 \\
\hline $\mathrm{H}$ & 3.963504120 & -4.107307930 & 1.075099690 \\
\hline $\mathrm{H}$ & 2.444847910 & -3.801382410 & 1.935019840 \\
\hline $\mathrm{H}$ & 0.184116670 & 6.114728070 & 2.059067090 \\
\hline \multicolumn{4}{|c|}{ C2-C3-C4-C5 angle: 120} \\
\hline C & -0.198637940 & 3.837416110 & 1.043653820 \\
\hline C & -0.088065640 & 2.591557690 & 0.465116970 \\
\hline C & 1.084471210 & 1.816232660 & 0.627644790 \\
\hline C & 2.149508510 & 2.348106030 & 1.410727250 \\
\hline C & 2.038068320 & 3.585827520 & 1.989793490 \\
\hline C & 0.861364300 & 4.331667910 & 1.804830900 \\
\hline $\mathrm{H}$ & -1.089543510 & 4.427348500 & 0.918937720 \\
\hline $\mathrm{H}$ & -0.903212560 & 2.200079680 & -0.115335270 \\
\hline $\mathrm{H}$ & 3.028587010 & 1.745030690 & 1.520405340 \\
\hline $\mathrm{H}$ & 2.823131000 & 4.011748780 & 2.584522630 \\
\hline 0 & 0.815120670 & 5.521026650 & 2.383611040 \\
\hline C & 1.180407310 & 0.539795500 & 0.058915050 \\
\hline C & 2.345779350 & -0.304921930 & 0.080893610 \\
\hline $\mathrm{H}$ & 0.306988280 & 0.276708680 & -0.540128600 \\
\hline $\mathrm{H}$ & 2.789421450 & -0.375042090 & -0.906848150 \\
\hline C & 2.148868480 & -1.551844510 & 0.719914130 \\
\hline 0 & 1.282804200 & -1.807839870 & 1.536106870 \\
\hline 0 & 3.053658580 & -2.488372310 & 0.364623000 \\
\hline
\end{tabular}




\begin{tabular}{|c|c|c|c|}
\hline C & 2.951204930 & -3.731971350 & 1.008101910 \\
\hline $\mathrm{H}$ & 2.000188240 & -4.205387470 & 0.802677020 \\
\hline $\mathrm{H}$ & 3.754472380 & -4.339479820 & 0.613736920 \\
\hline $\mathrm{H}$ & 3.061052230 & -3.630806700 & 2.079503040 \\
\hline $\mathrm{H}$ & -0.007150380 & 5.966533370 & 2.222679980 \\
\hline \multicolumn{4}{|c|}{ C2-C3-C4-C5 angle: 110} \\
\hline C & -0.286018430 & 3.700753860 & 1.248754920 \\
\hline C & -0.136312600 & 2.508919910 & 0.574552880 \\
\hline C & 1.072968380 & 1.760084760 & 0.679144960 \\
\hline C & 2.123462900 & 2.263574430 & 1.502342270 \\
\hline C & 1.970515370 & 3.456054760 & 2.178291880 \\
\hline C & 0.769184420 & 4.173658020 & 2.052333030 \\
\hline $\mathrm{H}$ & -1.197925610 & 4.265837940 & 1.171048650 \\
\hline $\mathrm{H}$ & -0.934059740 & 2.129321140 & -0.036265830 \\
\hline $\mathrm{H}$ & 3.017332100 & 1.676780770 & 1.566615780 \\
\hline $\mathrm{H}$ & 2.744903250 & 3.854088420 & 2.805197670 \\
\hline 0 & 0.679882870 & 5.322323670 & 2.726566190 \\
\hline C & 1.211723250 & 0.559552980 & 0.012694070 \\
\hline C & 2.384156680 & -0.283468630 & 0.026617190 \\
\hline $\mathrm{H}$ & 0.336240510 & 0.295935630 & -0.582066470 \\
\hline $\mathrm{H}$ & 2.933064740 & -0.324320180 & -0.900388250 \\
\hline C & 2.174786550 & -1.476103670 & 0.739040160 \\
\hline 0 & 1.262757330 & -1.672419860 & 1.527971170 \\
\hline 0 & 3.099320850 & -2.431054710 & 0.506546970 \\
\hline C & 2.963418260 & -3.623537450 & 1.235142370 \\
\hline $\mathrm{H}$ & 2.025428900 & -4.115776330 & 1.014927980 \\
\hline $\mathrm{H}$ & 3.787428580 & -4.254769380 & 0.931180420 \\
\hline $\mathrm{H}$ & 3.015271310 & -3.442338950 & 2.300417020 \\
\hline $\mathrm{H}$ & -0.161401030 & 5.741575270 & 2.603343240 \\
\hline \multicolumn{4}{|c|}{ C2-C3-C4-C5 angle: 100} \\
\hline C & -0.358680530 & 3.597737080 & 1.415945180 \\
\hline C & -0.186161300 & 2.459096690 & 0.660091910 \\
\hline C & 1.025405670 & 1.710786700 & 0.735739650 \\
\hline C & 2.054254100 & 2.158243700 & 1.616438700 \\
\hline C & 1.880283200 & 3.298557210 & 2.371783480 \\
\hline C & 0.676376210 & 4.016790670 & 2.273331120 \\
\hline $\mathrm{H}$ & -1.273146780 & 4.161304070 & 1.360820100 \\
\hline $\mathrm{H}$ & -0.968110450 & 2.120576960 & 0.006265480 \\
\hline $\mathrm{H}$ & 2.948351270 & 1.570548820 & 1.664669400 \\
\hline $\mathrm{H}$ & 2.637506920 & 3.652518600 & 3.044399400 \\
\hline 0 & 0.564447120 & 5.110749340 & 3.029503380 \\
\hline C & 1.182484450 & 0.562238330 & -0.013319620 \\
\hline C & 2.339036720 & -0.300529180 & -0.014630400 \\
\hline $\mathrm{H}$ & 0.307664650 & 0.326822450 & -0.620544770 \\
\hline
\end{tabular}




\begin{tabular}{lrrr} 
H & 3.016874030 & -0.260783710 & -0.846655340 \\
C & 2.172722330 & -1.439735910 & 0.771674750 \\
O & 1.246814190 & -1.624507750 & 1.552441710 \\
C & 3.148555070 & -2.364778150 & 0.632107220 \\
H & 3.040129170 & -3.511511270 & 1.433790800 \\
H & 2.132137720 & -4.059311300 & 1.219170250 \\
H & 3.900086580 & -4.123783850 & 1.197439770 \\
H & 3.049127000 & -3.260361220 & 2.486181220 \\
C2-C3-C4-C5 angle: 9 & -90 & & 2.923158800 \\
C & -0.429629750 & 3.507716300 & 1.552178700 \\
C & -0.238368700 & 2.421555510 & 0.726511590 \\
C & 0.962765680 & 1.656893190 & 0.793600080 \\
C & 1.961995950 & 2.032196680 & 1.738929320 \\
C & 1.770979010 & 3.120363700 & 2.563107890 \\
C & 0.576832750 & 3.856202260 & 2.472168410 \\
H & -1.337366040 & 4.082603390 & 1.503727040 \\
H & -0.998513070 & 2.136962080 & 0.023204820 \\
H & 2.848703330 & 1.433460310 & 1.783977790 \\
H & 2.505332380 & 3.417996630 & 3.286380770 \\
O & 0.446345090 & 4.895397370 & 3.298844890 \\
C & 1.131543390 & 0.556568360 & -0.024005910 \\
C & 2.264744270 & -0.334052580 & -0.042601380 \\
H & 0.265687450 & 0.365319100 & -0.658291750 \\
H & 3.058544040 & -0.188955030 & -0.748793580 \\
C & 2.161721160 & -1.421036400 & 0.815529060 \\
O & 1.236003110 & -1.622955880 & 1.594349740 \\
O & 3.203826200 & -2.283509220 & 0.750172130 \\
C & 3.148078410 & -3.392926730 & 1.606943140 \\
H & 2.281498430 & -4.007604900 & 1.402469410 \\
H & 4.049973260 & -3.960629190 & 1.420011440 \\
H & 3.116572540 & -3.090601440 & 2.645577820 \\
OMpCA0 & -0.390019220 & 5.329475550 & 3.194522250 \\
\hline
\end{tabular}

\section{OMpCA(W1)}

\section{C2-C3-C4-C5 angle: 180}

$\begin{array}{llll}\text { C } & -2.745526080 & 0.805613240 & -0.000471260 \\ \text { C } & -1.347170730 & 1.093763910 & 0.016773030 \\ \text { C } & -0.364320990 & 0.032673690 & -0.004471820 \\ \text { C } & -0.829918970 & -1.331504540 & -0.043720980 \\ \text { C } & -2.238391410 & -1.615774580 & -0.060935870 \\ \text { C } & -3.177807940 & -0.550695870 & -0.039267420 \\ \text { H } & -3.465546810 & 1.601310500 & 0.015581580 \\ \text { H } & -1.013941710 & 2.113656790 & 0.046008720 \\ \text { H } & -0.131065300 & -2.142744610 & -0.060350840\end{array}$




\begin{tabular}{|c|c|c|c|}
\hline $\mathrm{H}$ & -2.596175890 & -2.625919950 & -0.090081330 \\
\hline 0 & -4.481908720 & -0.882285320 & -0.057012280 \\
\hline C & 1.034584010 & 0.387624590 & 0.014566870 \\
\hline C & 2.081401490 & -0.442255360 & -0.000033880 \\
\hline $\mathrm{H}$ & 1.256137120 & 1.440598860 & 0.043835560 \\
\hline $\mathrm{H}$ & 1.990514880 & -1.510043260 & -0.028769370 \\
\hline C & 3.452887180 & 0.101509030 & 0.023786870 \\
\hline 0 & 3.742637950 & 1.258700720 & 0.056237450 \\
\hline 0 & 4.357365560 & -0.870161440 & 0.004507000 \\
\hline C & 5.716475720 & -0.479637700 & 0.024207470 \\
\hline $\mathrm{H}$ & 5.938819270 & 0.080122830 & 0.921385580 \\
\hline $\mathrm{H}$ & 6.287226050 & -1.395205270 & 0.004088400 \\
\hline $\mathrm{H}$ & 5.951033800 & 0.126423420 & -0.839140410 \\
\hline $\mathrm{H}$ & -5.038321240 & -0.108532070 & -0.040357760 \\
\hline 0 & -6.301158140 & 1.375238780 & -0.009590940 \\
\hline $\mathrm{H}$ & -6.871043360 & 1.423028840 & 0.746918870 \\
\hline $\mathrm{H}$ & -6.860999300 & 1.463079160 & -0.770000110 \\
\hline \multicolumn{4}{|c|}{ C2-C3-C4-C5 angle: 170} \\
\hline C & -2.736511240 & 0.815607020 & -0.184442050 \\
\hline C & -1.342674640 & 1.096642220 & -0.053886570 \\
\hline C & -0.361194510 & 0.034021830 & -0.061946410 \\
\hline C & -0.824217180 & -1.324762450 & -0.199019690 \\
\hline C & -2.228317410 & -1.601932180 & -0.327879480 \\
\hline C & -3.166141300 & -0.535326830 & -0.319651640 \\
\hline $\mathrm{H}$ & -3.455172830 & 1.612695900 & -0.179715510 \\
\hline $\mathrm{H}$ & -1.011689290 & 2.112391570 & 0.049882550 \\
\hline $\mathrm{H}$ & -0.126094680 & -2.136711040 & -0.212025980 \\
\hline $\mathrm{H}$ & -2.583848500 & -2.607943990 & -0.431804550 \\
\hline 0 & -4.466117870 & -0.860214450 & -0.444348990 \\
\hline C & 1.032975210 & 0.381751420 & 0.070664380 \\
\hline C & 2.081675420 & -0.446247800 & 0.047810540 \\
\hline $\mathrm{H}$ & 1.253984070 & 1.433897160 & 0.125506360 \\
\hline $\mathrm{H}$ & 1.990901280 & -1.512969930 & -0.009749670 \\
\hline C & 3.450212440 & 0.100464410 & -0.022805840 \\
\hline 0 & 3.740689520 & 1.257563720 & 0.006964950 \\
\hline 0 & 4.350469650 & -0.868352770 & -0.139377700 \\
\hline C & 5.705360330 & -0.474341820 & -0.234771150 \\
\hline $\mathrm{H}$ & 6.006737470 & 0.069718300 & 0.648881720 \\
\hline $\mathrm{H}$ & 6.273068550 & -1.387782130 & -0.322883610 \\
\hline $\mathrm{H}$ & 5.860658400 & 0.147898140 & -1.104485580 \\
\hline $\mathrm{H}$ & -5.021838310 & -0.085970660 & -0.427563590 \\
\hline 0 & -6.282301810 & 1.400530600 & -0.407068080 \\
\hline $\mathrm{H}$ & -6.901657830 & 1.412233630 & 0.310992410 \\
\hline $\mathrm{H}$ & -6.789609360 & 1.530250260 & -1.197739760 \\
\hline
\end{tabular}




\section{C2-C3-C4-C5 angle: 160}

\begin{tabular}{|c|c|c|c|}
\hline C & -2.711721040 & 0.836157880 & -0.364697740 \\
\hline C & -1.329982950 & 1.101505820 & -0.121197630 \\
\hline C & -0.353000760 & 0.034550090 & -0.115116920 \\
\hline C & -0.810090570 & -1.313300400 & -0.3478378 \\
\hline C & -2.202698910 & -1.574754060 & -0.587340100 \\
\hline C & -3.135195730 & -0.503691250 & -0.594493770 \\
\hline $\mathrm{H}$ & -3.426236020 & 1.636930820 & -0.372620740 \\
\hline $\mathrm{H}$ & -1.004202080 & 2.108798040 & 0.055517100 \\
\hline $\mathrm{H}$ & -0.114778420 & -2.127745240 & -0.353294630 \\
\hline $\mathrm{H}$ & -2.553126750 & -2.572344660 & -0.764216650 \\
\hline 0 & -4.424194550 & -0.813695560 & -0.825594490 \\
\hline C & 1.028924740 & 0.366361310 & 0.129212170 \\
\hline C & 2.083043420 & -0.456255220 & 0.094127160 \\
\hline $\mathrm{H}$ & 1.248344560 & 1.417154730 & 0.214252180 \\
\hline $\mathrm{H}$ & 1.991821640 & -1.520848800 & 0.002350480 \\
\hline C & 3.441494460 & 0.094809280 & -0.069240170 \\
\hline 0 & 3.734665200 & 1.251235920 & -0.036427580 \\
\hline 0 & 4.327247260 & -0.869740590 & -0.288114730 \\
\hline C & 5.667909450 & -0.471028970 & -0.497401530 \\
\hline $\mathrm{H}$ & 6.048213840 & 0.054330910 & 0.366867310 \\
\hline $\mathrm{H}$ & 6.225203010 & -1.381247970 & -0.656583080 \\
\hline $\mathrm{H}$ & 5.743655810 & 0.170198780 & -1.363897980 \\
\hline $\mathrm{H}$ & -4.977217040 & -0.037440080 & -0.811107460 \\
\hline 0 & -6.229481630 & 1.454870670 & -0.807737790 \\
\hline $\mathrm{H}$ & -6.896651660 & 1.439338250 & -0.133933730 \\
\hline $\mathrm{H}$ & -6.680430130 & 1.625358580 & -1.624401250 \\
\hline \multicolumn{4}{|c|}{ C2-C3-C4-C5 angle: 150} \\
\hline C & -2.692415120 & 0.829588830 & -0.328623440 \\
\hline C & -1.324763650 & 1.090177990 & -0.011611640 \\
\hline C & -0.346983830 & 0.023881050 & 0.015999010 \\
\hline C & -0.791991320 & -1.319301460 & -0.263896020 \\
\hline C & -2.171438020 & -1.575569510 & -0.575093630 \\
\hline C & -3.103208430 & -0.504754580 & -0.605981850 \\
\hline $\mathrm{H}$ & -3.406280630 & 1.630562090 & -0.354858260 \\
\hline $\mathrm{H}$ & -1.009222720 & 2.093546750 & 0.201881610 \\
\hline $\mathrm{H}$ & -0.095359160 & -2.132595560 & -0.256051790 \\
\hline $\mathrm{H}$ & -2.511882410 & -2.569320300 & -0.789177750 \\
\hline 0 & -4.379271340 & -0.809817350 & -0.905668110 \\
\hline C & 1.020178900 & 0.350689960 & 0.329350040 \\
\hline C & 2.087900980 & -0.457224830 & 0.2853138 \\
\hline $\mathrm{H}$ & 1.231878460 & 1.404062770 & 0.4083768 \\
\hline II & 2.001348120 & -1.523141780 & 0.1987712 \\
\hline$c$ & 3.419793460 & 0.099639540 & -0.0150429 \\
\hline
\end{tabular}




\begin{tabular}{|c|c|c|c|}
\hline 0 & 3.707329770 & 1.257919880 & -0.032856270 \\
\hline 0 & 4.284397270 & -0.864484340 & -0.308521530 \\
\hline C & 5.591888220 & -0.462037440 & -0.667170640 \\
\hline $\mathrm{H}$ & 6.057926770 & 0.082532450 & 0.141406280 \\
\hline $\mathrm{H}$ & 6.136160940 & -1.372006060 & -0.867602790 \\
\hline $\mathrm{H}$ & 5.569827070 & 0.162331200 & -1.548881950 \\
\hline $\mathrm{H}$ & -4.932696010 & -0.033746390 & -0.903801900 \\
\hline 0 & -6.181512360 & 1.461526760 & -0.938044640 \\
\hline $\mathrm{H}$ & -6.887575540 & 1.432827780 & -0.305587280 \\
\hline $\mathrm{H}$ & -6.582514280 & 1.654220830 & -1.775555980 \\
\hline \multicolumn{4}{|c|}{ C2-C3-C4-C5 angle: 140} \\
\hline C & -2.666409730 & 0.823329400 & -0.298955340 \\
\hline C & -1.316057100 & 1.080900630 & 0.087391230 \\
\hline C & -0.335983840 & 0.016878690 & 0.136466750 \\
\hline C & -0.765347600 & -1.321830890 & -0.187625750 \\
\hline C & -2.128620500 & -1.574396870 & -0.566567300 \\
\hline C & -3.060643990 & -0.505432040 & -0.620474370 \\
\hline $\mathrm{H}$ & -3.380085310 & 1.623683130 & -0.343331210 \\
\hline $\mathrm{H}$ & -1.013986760 & 2.080560590 & 0.334745760 \\
\hline $\mathrm{H}$ & -0.066373500 & -2.132874540 & -0.166401690 \\
\hline $\mathrm{H}$ & -2.455771580 & -2.564593560 & -0.815118040 \\
\hline 0 & -4.320792190 & -0.807075240 & -0.984502860 \\
\hline C & 1.011992200 & 0.339505370 & 0.516033050 \\
\hline C & 2.098075150 & -0.448675710 & 0.465592800 \\
\hline $\mathrm{H}$ & 1.212762350 & 1.396197590 & 0.588919280 \\
\hline $\mathrm{H}$ & 2.018081680 & -1.516534410 & 0.388757300 \\
\hline C & 3.391403680 & 0.108823470 & 0.031394600 \\
\hline 0 & 3.668384190 & 1.267489440 & -0.044998380 \\
\hline 0 & 4.229328080 & -0.859252080 & -0.321581390 \\
\hline C & 5.490440080 & -0.459830260 & -0.821692730 \\
\hline $\mathrm{H}$ & 6.032051740 & 0.108227450 & -0.079076910 \\
\hline $\mathrm{H}$ & 6.018343590 & -1.372148470 & -1.052941230 \\
\hline $\mathrm{H}$ & 5.374341680 & 0.140870990 & -1.712412390 \\
\hline $\mathrm{H}$ & -4.875805870 & -0.032194690 & -0.993549920 \\
\hline 0 & -6.123790780 & 1.462662450 & -1.053682160 \\
\hline $\mathrm{H}$ & -6.858215460 & 1.420157600 & -0.455212270 \\
\hline $\mathrm{H}$ & -6.485805050 & 1.675060250 & -1.904106240 \\
\hline \multicolumn{4}{|c|}{ C2-C3-C4-C5 angle: 130} \\
\hline C & -2.516028060 & 1.115696050 & -1.198683850 \\
\hline C & -1.248340970 & 1.219389560 & -0.681122060 \\
\hline C & -0.276412240 & 0.209587290 & -0.896196810 \\
\hline C & -0.649959500 & -0.934043970 & -1.677339320 \\
\hline C & -1.907139520 & -1.035950480 & -2.201662760 \\
\hline$C$ & -2.852747930 & -0.005853870 & -1.962247950 \\
\hline
\end{tabular}




\begin{tabular}{|c|c|c|c|}
\hline $\mathrm{H}$ & -3.251490750 & 1.879877710 & -1.028827120 \\
\hline $\mathrm{H}$ & -0.979981580 & 2.082753550 & -0.100023320 \\
\hline $\mathrm{H}$ & 0.092683490 & -1.694709590 & -1.812526850 \\
\hline $\mathrm{H}$ & -2.217660350 & -1.880523640 & -2.786277130 \\
\hline 0 & -4.047112510 & -0.170563920 & -2.482227050 \\
\hline C & 1.002166090 & 0.339774350 & -0.392862040 \\
\hline U & 2.032018600 & -0.682673840 & -0.411522360 \\
\hline $\mathrm{H}$ & 1.115480860 & 1.217731540 & 0.245322510 \\
\hline $\mathrm{H}$ & 2.165711170 & -0.975606670 & 0.630213060 \\
\hline U & 3.305202960 & -0.262120760 & -0.918169590 \\
\hline 0 & 3.517969790 & 0.621487710 & -1.724604580 \\
\hline 0 & 4.333270540 & -0.993693590 & -0.420215330 \\
\hline C & 5.610320320 & -0.689428320 & -0.910039890 \\
\hline $\mathrm{H}$ & 5.889871880 & 0.332021900 & -0.683870500 \\
\hline $\mathrm{H}$ & 6.292153600 & -1.369583810 & -0.417230250 \\
\hline $\mathrm{H}$ & 5.667210940 & -0.830859290 & -1.981801060 \\
\hline H & -4.643093490 & 0.555838920 & -2.290858760 \\
\hline 0 & -5.838851460 & 1.947294410 & -1.977197260 \\
\hline $\mathrm{H}$ & -6.618470580 & 1.801437000 & -1.456418230 \\
\hline $\mathrm{H}$ & -6.095776440 & 2.514740570 & -2.693018710 \\
\hline \multicolumn{4}{|c|}{ C2-C3-C4-C5 angle: 120} \\
\hline C & -2.536743270 & 1.019349460 & -0.983596930 \\
\hline C & -1.288904170 & 1.143618740 & -0.427569940 \\
\hline C & -0.299355800 & 0.139768060 & -0.594799510 \\
\hline C & -0.635402360 & -1.019961180 & -1.372139200 \\
\hline C & -1.874309840 & -1.144127980 & -1.931967170 \\
\hline C & -2.837202820 & -0.119186360 & -1.739267500 \\
\hline $\mathrm{H}$ & -3.284618910 & 1.779065030 & -0.852721310 \\
\hline $\mathrm{H}$ & -1.047172570 & 2.018375100 & 0.148050540 \\
\hline $\mathrm{H}$ & 0.124042050 & -1.767878190 & -1.480735250 \\
\hline $\mathrm{H}$ & -2.156667230 & -1.997866900 & -2.517475700 \\
\hline 0 & -4.010015310 & -0.302156590 & -2.297371270 \\
\hline C & 0.956338170 & 0.289579530 & -0.047380300 \\
\hline C & 2.020181070 & -0.694546570 & -0.087811570 \\
\hline $\mathrm{H}$ & 1.057782200 & 1.198576420 & 0.547796560 \\
\hline $\mathrm{H}$ & 2.216753650 & -1.071605400 & 0.909365340 \\
\hline C & 3.198465860 & -0.237944880 & -0.742394640 \\
\hline 0 & 3.270759800 & 0.651396330 & -1.571617920 \\
\hline 0 & 4.311425690 & -0.927588490 & -0.382580960 \\
\hline C & 5.499633890 & -0.578836000 & -1.036882490 \\
\hline $\mathrm{H}$ & 5.768087860 & 0.453757810 & -0.851279960 \\
\hline $\mathrm{H}$ & 6.266347960 & -1.229744180 & -0.637765830 \\
\hline$H$ & 5.420886650 & -0.724581210 & -2.106839670 \\
\hline 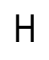 & -4.618416910 & 0.422710070 & -2.141057360 \\
\hline
\end{tabular}




\begin{tabular}{|c|c|c|c|}
\hline 0 & -5.833021350 & 1.806759010 & -1.895730690 \\
\hline $\mathrm{H}$ & -6.633408900 & 1.662640610 & -1.406878880 \\
\hline $\mathrm{H}$ & -6.061570850 & 2.366097820 & -2.627439870 \\
\hline \multicolumn{4}{|c|}{ C2-C3-C4-C5 angle: 110} \\
\hline C & -2.462736160 & 1.096390670 & -1.135065080 \\
\hline C & -1.253432690 & 1.180502620 & -0.494268920 \\
\hline C & -0.269525130 & 0.165972210 & -0.629206350 \\
\hline C & -0.569696150 & -0.961506720 & -1.466625110 \\
\hline C & -1.771642910 & -1.048665580 & -2.107267150 \\
\hline C & -2.729626720 & -0.013275410 & -1.945185680 \\
\hline $\mathrm{H}$ & -3.205166800 & 1.865511170 & -1.031201130 \\
\hline $\mathrm{H}$ & -1.036639230 & 2.032049310 & 0.124396930 \\
\hline $\mathrm{H}$ & 0.188207290 & -1.712904290 & -1.559534790 \\
\hline $\mathrm{H}$ & -2.025659210 & -1.875795300 & -2.741835520 \\
\hline 0 & -3.863142810 & -0.156364340 & -2.587829830 \\
\hline C & 0.946924050 & 0.278467610 & 0.007657060 \\
\hline C & 2.023354560 & -0.688862020 & -0.045450360 \\
\hline $\mathrm{H}$ & 1.034541100 & 1.186242090 & 0.606984720 \\
\hline $\mathrm{H}$ & 2.206345700 & -1.213900840 & 0.878313380 \\
\hline C & 3.146083110 & -0.245650270 & -0.776932330 \\
\hline 0 & 3.166580200 & 0.670611550 & -1.584014180 \\
\hline 0 & 4.272345660 & -0.968703480 & -0.535623320 \\
\hline C & 5.406118240 & -0.620816300 & -1.279410570 \\
\hline $\mathrm{H}$ & 5.711895040 & 0.400010600 & -1.086401780 \\
\hline $\mathrm{H}$ & 6.190968540 & -1.298297060 & -0.969018270 \\
\hline $\mathrm{H}$ & 5.233292250 & -0.729875650 & -2.342849380 \\
\hline $\mathrm{H}$ & -4.467695140 & 0.576331950 & -2.453634160 \\
\hline 0 & -5.665148850 & 1.980155530 & -2.256790760 \\
\hline $\mathrm{H}$ & -6.504242540 & 1.844310530 & -1.835105470 \\
\hline $\mathrm{H}$ & -5.821306540 & 2.570080250 & -2.983509150 \\
\hline \multicolumn{4}{|c|}{ C2-C3-C4-C5 angle: 100} \\
\hline C & -2.448474940 & 1.085802800 & -1.098575510 \\
\hline C & -1.268373900 & 1.174775860 & -0.406321200 \\
\hline C & -0.260706580 & 0.182332770 & -0.524232790 \\
\hline C & -0.505000070 & -0.929233170 & -1.400032540 \\
\hline C & -1.678489930 & -1.023984350 & -2.089758550 \\
\hline C & -2.661322730 & -0.008967030 & -1.944293650 \\
\hline $\mathrm{H}$ & -3.207714300 & 1.840151250 & -1.009292540 \\
\hline $\mathrm{H}$ & -1.092736370 & 2.014612760 & 0.240723750 \\
\hline $\mathrm{H}$ & 0.271550380 & -1.662013820 & -1.486428520 \\
\hline $\mathrm{H}$ & -1.889429530 & -1.838078860 & -2.756123230 \\
\hline 0 & -3.762567140 & -0.155130440 & -2.638973070 \\
\hline C & 0.926777300 & 0.305256840 & 0.165149290 \\
\hline C & 2.042156580 & -0.612807870 & 0.110829480 \\
\hline
\end{tabular}




\begin{tabular}{|c|c|c|c|}
\hline $\mathrm{H}$ & 0.978338230 & 1.215647950 & 0.764695330 \\
\hline $\mathrm{H}$ & 2.203889480 & -1.271403650 & 0.943681310 \\
\hline C & 3.095061690 & -0.213379580 & -0.722406900 \\
\hline 0 & 3.068272110 & 0.701885520 & -1.534195280 \\
\hline 0 & 4.219166090 & -0.970902120 & -0.583689740 \\
\hline C & 5.290198900 & -0.651020670 & -1.425369060 \\
\hline $\mathrm{H}$ & 5.639450070 & 0.360709430 & -1.260292650 \\
\hline $\mathrm{H}$ & 6.081713880 & -1.349898320 & -1.186869710 \\
\hline $\mathrm{H}$ & 5.021339540 & -0.752141100 & -2.469682570 \\
\hline $\mathrm{H}$ & -4.383940120 & 0.565783340 & -2.517388300 \\
\hline 0 & -5.605621740 & 1.950252130 & -2.350751530 \\
\hline $\mathrm{H}$ & -6.465314920 & 1.796699160 & -1.979632400 \\
\hline $\mathrm{H}$ & -5.727227110 & 2.557069980 & -3.070176600 \\
\hline \multicolumn{4}{|c|}{ C2-C3-C4-C5 angle: 90} \\
\hline C & -2.439425620 & 1.077792550 & -1.065822170 \\
\hline C & -1.287167130 & 1.180240870 & -0.329805120 \\
\hline C & -0.245132310 & 0.223977100 & -0.441316380 \\
\hline C & -0.423894730 & -0.866332790 & -1.358620620 \\
\hline C & -1.569283770 & -0.976165230 & -2.091356910 \\
\hline C & -2.588325660 & 0.003952880 & -1.950857610 \\
\hline $\mathrm{H}$ & -3.224178740 & 1.806082910 & -0.981275060 \\
\hline $\mathrm{H}$ & -1.160354630 & 2.004524310 & 0.347929750 \\
\hline $\mathrm{H}$ & 0.376850700 & -1.572476460 & -1.444304410 \\
\hline $\mathrm{H}$ & -1.730318230 & -1.773979920 & -2.790464860 \\
\hline 0 & -3.658521200 & -0.153786050 & -2.689255350 \\
\hline C & 0.915200050 & 0.367428220 & 0.291327700 \\
\hline C & 2.070896800 & -0.497118190 & 0.249688820 \\
\hline $\mathrm{H}$ & 0.918350900 & 1.269507940 & 0.904661700 \\
\hline $\mathrm{H}$ & 2.178385110 & -1.282581590 & 0.971823450 \\
\hline C & 3.058641420 & -0.171676550 & -0.680145710 \\
\hline 0 & 3.014107910 & 0.733477900 & -1.504338290 \\
\hline 0 & 4.150336740 & -0.988790600 & -0.622200420 \\
\hline C & 5.171785080 & -0.722588450 & -1.539998970 \\
\hline $\mathrm{H}$ & 5.578956840 & 0.272080960 & -1.407455640 \\
\hline $\mathrm{H}$ & 5.945103090 & -1.457055960 & -1.353740960 \\
\hline $\mathrm{H}$ & 4.825451980 & -0.814518710 & -2.562271730 \\
\hline $\mathrm{H}$ & -4.305641140 & 0.544762360 & -2.570493340 \\
\hline 0 & -5.571148120 & 1.888468360 & -2.411914130 \\
\hline $\mathrm{H}$ & -6.441393120 & 1.700720130 & -2.083559810 \\
\hline $\mathrm{H}$ & -5.678287330 & 2.516072860 & -3.115641120 \\
\hline
\end{tabular}

OMpCA(W2)

C2-C3-C4-C5 angle: 180

$\begin{array}{llll}\text { C } & -2.915205850 & 0.844513650 & -0.012838190 \\ \text { C } & -1.498681620 & 1.022703030 & -0.008808430\end{array}$




\begin{tabular}{|c|c|c|c|}
\hline C & -0.605726330 & -0.116205470 & 0.012660110 \\
\hline C & -1.178871190 & -1.439515280 & 0.032204400 \\
\hline C & -2.605991810 & -1.610452310 & 0.029437900 \\
\hline C & -3.452650500 & -0.472129930 & 0.006344340 \\
\hline $\mathrm{H}$ & -3.565510480 & 1.699780110 & -0.032439920 \\
\hline $\mathrm{H}$ & -1.081509220 & 2.011129290 & -0.030030340 \\
\hline $\mathrm{H}$ & -0.548556860 & -2.304859120 & 0.052676020 \\
\hline $\mathrm{H}$ & -3.045101810 & -2.588144670 & 0.043901940 \\
\hline 0 & -4.785719540 & -0.701185490 & 0.004210780 \\
\hline C & 0.814420780 & 0.134447950 & 0.011550790 \\
\hline C & 1.795094840 & -0.775190720 & 0.002363940 \\
\hline $\mathrm{H}$ & 1.099332840 & 1.170950210 & 0.019000380 \\
\hline $\mathrm{H}$ & 1.621959610 & -1.833053860 & -0.007748420 \\
\hline C & 3.204118080 & -0.349339940 & 0.002641050 \\
\hline 0 & 3.600118840 & 0.782713780 & 0.009161570 \\
\hline 0 & 4.024326550 & -1.386366930 & -0.004242010 \\
\hline C & 5.414286660 & -1.115689450 & -0.010814930 \\
\hline $\mathrm{H}$ & 5.697300270 & -0.562721210 & 0.873110620 \\
\hline $\mathrm{H}$ & 5.900470140 & -2.078502050 & -0.021310710 \\
\hline $\mathrm{H}$ & 5.686145720 & -0.548627970 & -0.889174310 \\
\hline 0 & 2.180608890 & 3.391325330 & -0.014098980 \\
\hline $\mathrm{H}$ & 2.764724400 & 2.649248480 & -0.138405280 \\
\hline $\mathrm{H}$ & 2.401211610 & 3.735001320 & 0.840593750 \\
\hline $\mathrm{H}$ & -5.266914180 & 0.113932070 & -0.018397330 \\
\hline \multicolumn{4}{|c|}{ C2-C3-C4-C5 angle: 170} \\
\hline C & -0.224902590 & 3.760114980 & 0.297163230 \\
\hline C & -0.283073520 & 2.337911780 & 0.186779000 \\
\hline C & 0.926889720 & 1.549258790 & 0.091529880 \\
\hline C & 2.196555510 & 2.233327280 & 0.100885180 \\
\hline C & 2.246277260 & 3.665863790 & 0.207305160 \\
\hline C & 1.041056150 & 4.408094120 & 0.305103850 \\
\hline $\mathrm{H}$ & -1.131605770 & 4.331608130 & 0.374990050 \\
\hline $\mathrm{H}$ & -1.232166950 & 1.837246330 & 0.188448150 \\
\hline $\mathrm{H}$ & 3.111593570 & 1.682286750 & 0.025564560 \\
\hline $\mathrm{H}$ & 3.183003610 & 4.186823350 & 0.215296300 \\
\hline 0 & 1.156621060 & 5.752102510 & 0.404480170 \\
\hline C & 0.796985580 & 0.116817130 & -0.007213970 \\
\hline C & 1.784603960 & -0.785782920 & -0.025143450 \\
\hline $\mathrm{H}$ & -0.211894720 & -0.254386480 & 0.001900610 \\
\hline $\mathrm{H}$ & 2.824246860 & -0.524070790 & -0.027907020 \\
\hline C & 1.480722450 & -2.221269660 & 0.090039630 \\
\hline 0 & 0.386724610 & -2.710951980 & 0.134782860 \\
\hline 0 & 2.584292950 & -2.946872740 & 0.152654960 \\
\hline C & 2.433621600 & -4.348356140 & 0.288662240 \\
\hline
\end{tabular}




\begin{tabular}{|c|c|c|c|}
\hline $\mathrm{H}$ & 1.898862540 & -4.756210100 & -0.556831150 \\
\hline $\mathrm{H}$ & 3.434586250 & -4.748541210 & 0.326688850 \\
\hline $\mathrm{H}$ & 1.899932960 & -4.586116770 & 1.197324460 \\
\hline 0 & -2.329002720 & -1.507898710 & 0.182278670 \\
\hline $\mathrm{H}$ & -1.537440880 & -2.023571960 & 0.304199740 \\
\hline $\mathrm{H}$ & -2.684629440 & -1.798670810 & -0.646131690 \\
\hline $\mathrm{H}$ & 0.304149420 & 6.158921950 & 0.469906930 \\
\hline \multicolumn{4}{|c|}{ C2-C3-C4-C5 angle: 160} \\
\hline C & -0.235294870 & 3.726767860 & 0.559830980 \\
\hline C & -0.292970490 & 2.316242420 & 0.346708090 \\
\hline C & 0.917310570 & 1.539081140 & 0.182996220 \\
\hline C & 2.185714020 & 2.224569070 & 0.223199010 \\
\hline C & 2.234181960 & 3.646119450 & 0.430654490 \\
\hline C & 1.029250000 & 4.375990810 & 0.597684140 \\
\hline $\mathrm{H}$ & -1.141918240 & 4.288480180 & 0.691378900 \\
\hline $\mathrm{H}$ & -1.240961820 & 1.813935190 & 0.326658950 \\
\hline $\mathrm{H}$ & 3.100623790 & 1.682014030 & 0.099790960 \\
\hline $\mathrm{H}$ & 3.170094270 & 4.167549420 & 0.464180390 \\
\hline 0 & 1.143707610 & 5.709755110 & 0.791901250 \\
\hline C & 0.788531000 & 0.117785080 & -0.013313670 \\
\hline C & 1.773284300 & -0.789025520 & -0.057910410 \\
\hline $\mathrm{H}$ & -0.219839850 & -0.254567550 & 0.012182610 \\
\hline $\mathrm{H}$ & 2.812452230 & -0.524829470 & -0.075873720 \\
\hline C & 1.480718120 & -2.212446510 & 0.172868540 \\
\hline 0 & 0.391284560 & -2.704516930 & 0.273317350 \\
\hline 0 & 2.590867860 & -2.921848510 & 0.285407110 \\
\hline C & 2.452902620 & -4.306143390 & 0.550056090 \\
\hline $\mathrm{H}$ & 1.911114440 & -4.792742100 & -0.247944580 \\
\hline $\mathrm{H}$ & 3.457424980 & -4.694337810 & 0.611434660 \\
\hline $\mathrm{H}$ & 1.932268470 & -4.463964580 & 1.483360170 \\
\hline 0 & -2.322275200 & -1.497073990 & 0.333472170 \\
\hline $\mathrm{H}$ & -1.526957620 & -2.007479460 & 0.453773320 \\
\hline $\mathrm{H}$ & -2.710072910 & -1.832622950 & -0.462978910 \\
\hline $\mathrm{H}$ & 0.291401770 & 6.108576050 & 0.896836840 \\
\hline \multicolumn{4}{|c|}{ C2-C3-C4-C5 angle: 150} \\
\hline C & -0.252777050 & 3.674597660 & 0.812280620 \\
\hline C & -0.308753480 & 2.283068580 & 0.497738930 \\
\hline C & 0.902822490 & 1.522901800 & 0.272672240 \\
\hline C & 2.169308390 & 2.209103100 & 0.350263470 \\
\hline C & 2.214890880 & 3.612772220 & 0.657191810 \\
\hline C & 1.009513140 & 4.324280580 & 0.885826870 \\
\hline $\mathrm{H}$ & -1.159988890 & 4.221598610 & 0.992660300 \\
\hline $\mathrm{H}$ & -1.255125210 & 1.779430100 & 0.452798820 \\
\hline $\mathrm{H}$ & 3.084670970 & 1.679119080 & 0.183029920 \\
\hline
\end{tabular}




\begin{tabular}{|c|c|c|c|}
\hline $\mathrm{H}$ & 3.149497130 & 4.133858220 & 0.719831760 \\
\hline 0 & 1.121443670 & 5.641324490 & 1.173632530 \\
\hline C & 0.776848260 & 0.120071690 & -0.019750780 \\
\hline C & 1.756868500 & -0.793959340 & -0.085143900 \\
\hline $\mathrm{H}$ & -0.230991880 & -0.253904030 & 0.015700730 \\
\hline $\mathrm{H}$ & 2.795347640 & -0.526075910 & -0.114331780 \\
\hline C & 1.481662040 & -2.197439570 & 0.257270580 \\
\hline 0 & 0.399443940 & -2.691868520 & 0.412637830 \\
\hline 0 & 2.601695900 & -2.882267710 & 0.416149230 \\
\hline C & 2.482986580 & -4.239211550 & 0.803403710 \\
\hline $\mathrm{H}$ & 1.937098910 & -4.799900110 & 0.058631500 \\
\hline $\mathrm{H}$ & 3.492681130 & -4.609815060 & 0.885353210 \\
\hline $\mathrm{H}$ & 1.975823310 & -4.319665790 & 1.753847440 \\
\hline 0 & -2.312070730 & -1.480281010 & 0.484348570 \\
\hline $\mathrm{H}$ & -1.512083760 & -1.983563370 & 0.604508760 \\
\hline $\mathrm{H}$ & -2.731074230 & -1.863768080 & -0.273723000 \\
\hline $\mathrm{H}$ & 0.268791170 & 6.028617600 & 1.313150190 \\
\hline \multicolumn{4}{|c|}{ C2-C3-C4-C5 angle: 140} \\
\hline C & -0.279043680 & 3.606530600 & 1.046919830 \\
\hline C & -0.330398140 & 2.240579280 & 0.634397950 \\
\hline C & 0.8845888360 & 1.500909970 & 0.361442650 \\
\hline C & 2.148094710 & 2.185949260 & 0.487997190 \\
\hline C & 2.187495080 & 3.565348400 & 0.891667080 \\
\hline C & 0.979728560 & 4.254542620 & 1.167453640 \\
\hline $\mathrm{H}$ & -1.188693990 & 4.135164180 & 1.265747790 \\
\hline $\mathrm{H}$ & -1.274498160 & 1.736662000 & 0.557796650 \\
\hline $\mathrm{H}$ & 3.065590390 & 1.671000310 & 0.288478810 \\
\hline $\mathrm{H}$ & 3.120187520 & 4.084059120 & 0.991653220 \\
\hline 0 & 1.086174410 & 5.548865450 & 1.546565420 \\
\hline C & 0.764754120 & 0.123950170 & -0.024428550 \\
\hline C & 1.738761550 & -0.800418440 & -0.105194730 \\
\hline $\mathrm{H}$ & -0.242833610 & -0.251833560 & 0.013395480 \\
\hline $\mathrm{H}$ & 2.776458650 & -0.528044380 & -0.143737340 \\
\hline C & 1.485339360 & -2.176422320 & 0.344095750 \\
\hline 0 & 0.412558400 & -2.670950640 & 0.557149160 \\
\hline 0 & 2.617254990 & -2.832006110 & 0.539980310 \\
\hline C & 2.522373680 & -4.152851560 & 1.041510740 \\
\hline $\mathrm{H}$ & 1.971637310 & -4.780394440 & 0.356046740 \\
\hline $\mathrm{H}$ & 3.538051100 & -4.503487660 & 1.136550440 \\
\hline $\mathrm{H}$ & 2.032081540 & -4.159181200 & 2.004120470 \\
\hline 0 & -2.296237310 & -1.453671800 & 0.635936660 \\
\hline $\mathrm{H}$ & -1.490667230 & -1.948110010 & 0.756632360 \\
\hline $\mathrm{H}$ & -2.746692700 & -1.888530610 & -0.074919520 \\
\hline $\mathrm{H}$ & 0.231876250 & 5.922248680 & 1.711566260 \\
\hline
\end{tabular}




\section{C2-C3-C4-C5 angle: 130}

\begin{tabular}{|c|c|c|c|}
\hline C & -0.273526770 & 3.551729040 & 1.062760360 \\
\hline C & -0.331933030 & 2.211212290 & 0.574154420 \\
\hline C & 0.880162890 & 1.476239810 & 0.272840210 \\
\hline C & 2.146513540 & 2.146156380 & 0.448323000 \\
\hline C & 2.190968100 & 3.501801750 & 0.926634750 \\
\hline C & 0.986756330 & 4.183777100 & 1.228963750 \\
\hline $\mathrm{H}$ & -1.181872970 & 4.072338690 & 1.305095630 \\
\hline $\mathrm{H}$ & -1.278573980 & 1.717765210 & 0.467969080 \\
\hline $\mathrm{H}$ & 3.062332260 & 1.634602100 & 0.232938690 \\
\hline $\mathrm{H}$ & 3.126646720 & 4.006783590 & 1.062782100 \\
\hline 0 & 1.098638080 & 5.454915770 & 1.679137600 \\
\hline C & 0.755500890 & 0.129705300 & -0.187330920 \\
\hline C & 1.713330070 & -0.819293140 & -0.271801180 \\
\hline $\mathrm{H}$ & -0.256117820 & -0.236851010 & -0.149280370 \\
\hline $\mathrm{H}$ & 2.752763390 & -0.552996760 & -0.325510190 \\
\hline C & 1.473170210 & -2.152130670 & 0.294154200 \\
\hline 0 & 0.407741840 & -2.628376110 & 0.577722390 \\
\hline 0 & 2.612029050 & -2.787679340 & 0.517333740 \\
\hline C & 2.532708860 & -4.058789640 & 1.135985160 \\
\hline $\mathrm{H}$ & 1.965466530 & -4.744446150 & 0.523562580 \\
\hline $\mathrm{H}$ & 3.551274470 & -4.399971150 & 1.234932030 \\
\hline $\mathrm{H}$ & 2.067907630 & -3.979990760 & 2.108011010 \\
\hline 0 & -2.286747700 & -1.380774090 & 0.644727890 \\
\hline $\mathrm{H}$ & -1.478187750 & -1.868563820 & 0.773793560 \\
\hline $\mathrm{H}$ & -2.768529440 & -1.872321530 & -0.006065090 \\
\hline $\mathrm{H}$ & 0.245519780 & 5.825064450 & 1.856990050 \\
\hline \multicolumn{4}{|c|}{ C2-C3-C4-C5 angle: 120} \\
\hline C & -0.272369470 & 3.597564090 & 0.915028010 \\
\hline C & -0.371065930 & 2.279532260 & 0.375325250 \\
\hline C & 0.804375050 & 1.432007780 & 0.216471970 \\
\hline C & 2.094698320 & 2.022453430 & 0.612878710 \\
\hline C & 2.154232010 & 3.369935220 & 1.123310590 \\
\hline C & 0.990764450 & 4.124437200 & 1.271952510 \\
\hline $\mathrm{H}$ & -1.164658350 & 4.180369630 & 1.055220620 \\
\hline $\mathrm{H}$ & -1.331175200 & 1.872623720 & 0.124641740 \\
\hline $\mathrm{H}$ & 2.994365120 & 1.447836780 & 0.536880590 \\
\hline $\mathrm{H}$ & 3.096200410 & 3.802041620 & 1.399976550 \\
\hline 0 & 1.120109860 & 5.378639490 & 1.770238880 \\
\hline C & 0.659351720 & 0.189916980 & -0.273261450 \\
\hline C & 1.698514890 & -0.816148660 & -0.328430640 \\
\hline $\mathrm{H}$ & -0.342925720 & -0.154299640 & -0.455920810 \\
\hline $\mathrm{H}$ & 2.704596630 & -0.570567260 & -0.607583250 \\
\hline C & 1.520358080 & -2.087913760 & 0.3733176 \\
\hline
\end{tabular}




\begin{tabular}{|c|c|c|c|}
\hline 0 & 0.493523940 & -2.474969790 & 0.857616230 \\
\hline 0 & 2.642647530 & -2.787660780 & 0.421557450 \\
\hline C & 2.596972480 & -4.038535310 & 1.084506000 \\
\hline $\mathrm{H}$ & 1.893733400 & -4.699541270 & 0.598925470 \\
\hline $\mathrm{H}$ & 3.595464240 & -4.441217690 & 1.019255790 \\
\hline $\mathrm{H}$ & 2.311204190 & -3.909884670 & 2.118313500 \\
\hline 0 & -2.282196130 & -1.339028220 & 0.701928630 \\
\hline $\mathrm{H}$ & -1.421818570 & -1.680501770 & 0.925711440 \\
\hline $\mathrm{H}$ & -2.748407570 & -2.073661010 & 0.328015060 \\
\hline $\mathrm{H}$ & 0.277445780 & 5.806478920 & 1.822947970 \\
\hline \multicolumn{4}{|c|}{ C2-C3-C4-C5 angle: 110} \\
\hline C & -0.662265670 & 3.289902470 & 1.545962240 \\
\hline C & -0.506804690 & 2.093807940 & 0.887983100 \\
\hline C & 0.717989900 & 1.381200210 & 0.944176080 \\
\hline C & 1.793381400 & 1.928802450 & 1.721572420 \\
\hline C & 1.641171940 & 3.120384330 & 2.370928590 \\
\hline C & 0.402201650 & 3.803422220 & 2.282840340 \\
\hline $\mathrm{H}$ & -1.596538360 & 3.821620100 & 1.510080990 \\
\hline $\mathrm{H}$ & -1.327590920 & 1.667866990 & 0.343809630 \\
\hline $\mathrm{H}$ & 2.705065620 & 1.367813220 & 1.762225640 \\
\hline $\mathrm{H}$ & 2.424029690 & 3.561549730 & 2.956992410 \\
\hline 0 & 0.318946460 & 4.947753370 & 2.937994970 \\
\hline C & 0.840295690 & 0.169211580 & 0.299254500 \\
\hline C & 2.009331810 & -0.688431560 & 0.331377400 \\
\hline $\mathrm{H}$ & -0.035413690 & -0.100298380 & -0.284067610 \\
\hline $\mathrm{H}$ & 2.568116350 & -0.738477070 & -0.588614190 \\
\hline C & 1.843908370 & -1.879945210 & 1.052231230 \\
\hline 0 & 0.957585460 & -2.127266670 & 1.869535790 \\
\hline 0 & 2.803319880 & -2.804581850 & 0.811627940 \\
\hline C & 2.733266690 & -3.995883690 & 1.546919300 \\
\hline $\mathrm{H}$ & 1.812060830 & -4.530119000 & 1.351643600 \\
\hline $\mathrm{H}$ & 3.574475810 & -4.595514190 & 1.226410170 \\
\hline $\mathrm{H}$ & 2.804182990 & -3.809925190 & 2.610905500 \\
\hline 0 & -1.662210920 & -0.971959310 & 1.753006090 \\
\hline $\mathrm{H}$ & -0.797728380 & -1.348395870 & 1.928167780 \\
\hline $\mathrm{H}$ & -2.240449460 & -1.718887490 & 1.684970470 \\
\hline $\mathrm{H}$ & -0.537386460 & 5.348155010 & 2.851441090 \\
\hline \multicolumn{4}{|c|}{ C2-C3-C4-C5 angle: 100} \\
\hline C & -0.648660060 & 3.248955300 & 1.586893960 \\
\hline C & -0.517221870 & 2.083878360 & 0.870575130 \\
\hline C & 0.699425310 & 1.355827670 & 0.871989940 \\
\hline C & 1.794268170 & 1.855362910 & 1.655102620 \\
\hline C & 1.668067690 & 3.017468740 & 2.359834340 \\
\hline C & 0.434298320 & 3.716370130 & 2.327032730 \\
\hline
\end{tabular}




\begin{tabular}{|c|c|c|c|}
\hline $\mathrm{H}$ & -1.578289450 & 3.789775360 & 1.594670920 \\
\hline $\mathrm{H}$ & -1.352116980 & 1.692424790 & 0.322263140 \\
\hline $\mathrm{H}$ & 2.698788530 & 1.281632510 & 1.660708530 \\
\hline & 2.464744690 & 3.421080380 & 2.954214870 \\
\hline & 0.375111020 & 4.827048740 & 3.038852070 \\
\hline 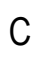 & 0.791960750 & 0.172511990 & 0.170290470 \\
\hline & 1.926696000 & -0.727552510 & 0.172387880 \\
\hline & -0.109644060 & -0.069819610 & -0.384627090 \\
\hline & 2.587414100 & -0.728129200 & -0.673965840 \\
\hline & 1.810268660 & -1.843199740 & 0.997477920 \\
\hline & 0.931207030 & -2.047536980 & 1.838618350 \\
\hline & 2.802247430 & -2.754474420 & 0.838040380 \\
\hline 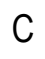 & 2.763021450 & -3.882387020 & 1.668298210 \\
\hline & 1.859987530 & -4.459285690 & 1.513738190 \\
\hline & 3.624279720 & -4.480473980 & 1.402083540 \\
\hline & 2.820736670 & -3.608724980 & 2.714111290 \\
\hline & -1.694833920 & -0.929279470 & 1.701530440 \\
\hline & -0.823638240 & -1.295140280 & 1.869333360 \\
\hline & -2.266007030 & -1.682834930 & 1.649348510 \\
\hline & -0.479169460 & 5.238306070 & 2.990571610 \\
\hline \multicolumn{4}{|c|}{ C2-C3-C4-C5 angle: 90} \\
\hline & -0.623175960 & 3.229188310 & 1.608532450 \\
\hline & -0.525229280 & 2.090534320 & 0.845452010 \\
\hline & 0.669358640 & 1.328922640 & 0.813288030 \\
\hline & 1.779820210 & 1.766795760 & 1.611116330 \\
\hline & 1.688911790 & 2.903167430 & 2.361118610 \\
\hline & 0.474162810 & 3.636575910 & 2.362152350 \\
\hline & -1.537912160 & 3.793799600 & 1.642079380 \\
\hline & -1.371978440 & 1.745689010 & 0.284191040 \\
\hline & 2.668661380 & 1.169381950 & 1.595068330 \\
\hline & 2.497623830 & 3.259274410 & 2.969412870 \\
\hline & 0.447952210 & 4.717580390 & 3.119368610 \\
\hline & 0.720867030 & 0.168984830 & 0.067525320 \\
\hline & 1.811711430 & -0.781652910 & 0.039041020 \\
\hline & -0.198238610 & -0.031018330 & -0.474290450 \\
\hline & 2.567725600 & -0.712940360 & -0.718247310 \\
\hline & 1.764955740 & -1.817247180 & 0.961127400 \\
\hline & 0.903933070 & -1.997123840 & 1.828233530 \\
\hline & 2.803330190 & -2.687684920 & 0.866138890 \\
\hline & 2.812974300 & -3.758301550 & 1.769372020 \\
\hline & 1.937088290 & -4.384230620 & 1.654528440 \\
\hline & 3.700689030 & -4.333729440 & 1.542480720 \\
\hline $\mathrm{H}$ & 2.856387650 & -3.414366090 & 2.795034490 \\
\hline & -1.728163510 & -0.911579000 & 1.656994250 \\
\hline
\end{tabular}




$\begin{array}{llrl}\mathrm{H} & -0.854257160 & -1.269518680 & 1.830159600 \\ \mathrm{H} & -2.288823680 & -1.671460490 & 1.585772860 \\ \mathrm{H} & -0.395432410 & 5.152763000 & 3.093724680\end{array}$

\section{OMpCA}

C1-C2-C3-C4 angle: 180

\begin{tabular}{|c|c|c|c|}
\hline C & -0.034919980 & 4.113935040 & 0.102789960 \\
\hline C & -0.052962520 & 2.686905840 & 0.065635540 \\
\hline C & 1.177597720 & 1.925844590 & 0.054950470 \\
\hline C & 2.428566700 & 2.643167990 & 0.082772340 \\
\hline C & 2.440232470 & 4.079780430 & 0.120130800 \\
\hline C & 1.214156250 & 4.793976470 & 0.129631720 \\
\hline $\mathrm{H}$ & -0.957263650 & 4.665289120 & 0.110300600 \\
\hline $\mathrm{H}$ & -0.991272770 & 2.166851050 & 0.045231770 \\
\hline $\mathrm{H}$ & 3.358621870 & 2.112919800 & 0.075869020 \\
\hline $\mathrm{H}$ & 3.362820880 & 4.624912630 & 0.141068650 \\
\hline 0 & 1.292385390 & 6.143421540 & 0.165214820 \\
\hline C & 1.095796980 & 0.484589280 & 0.016957690 \\
\hline C & 2.110975800 & -0.382531180 & 0.001935520 \\
\hline $\mathrm{H}$ & 0.104497710 & 0.065671050 & -0.001206330 \\
\hline $\mathrm{H}$ & 3.142009650 & -0.089195420 & 0.017131200 \\
\hline C & 1.839137830 & -1.833642540 & -0.037710560 \\
\hline 0 & 0.757514830 & -2.336337920 & -0.058692270 \\
\hline 0 & 2.965328570 & -2.534433830 & -0.047627320 \\
\hline C & 2.842595780 & -3.943585750 & -0.085093610 \\
\hline $\mathrm{H}$ & 2.320598880 & -4.255995990 & -0.978076370 \\
\hline $\mathrm{H}$ & 3.850895200 & -4.327575080 & -0.087656330 \\
\hline $\mathrm{H}$ & 2.307671660 & -4.301687470 & 0.782730200 \\
\hline $\mathrm{H}$ & 0.428545080 & 6.531005100 & 0.168915910 \\
\hline \multicolumn{4}{|c|}{ C1-C2-C3-C4 angle: 170} \\
\hline C & -0.028414690 & 4.116981540 & -0.012551580 \\
\hline C & -0.052621970 & 2.689455880 & -0.014389390 \\
\hline C & 1.170602890 & 1.924207440 & 0.089717120 \\
\hline C & 2.419761690 & 2.636014850 & 0.203954260 \\
\hline C & 2.437354400 & 4.072788890 & 0.210203530 \\
\hline C & 1.218923080 & 4.792000050 & 0.099366010 \\
\hline $\mathrm{H}$ & -0.944351970 & 4.672516960 & -0.097425100 \\
\hline $\mathrm{H}$ & -0.989000980 & 2.172807950 & -0.101650730 \\
\hline $\mathrm{H}$ & 3.340404560 & 2.100224930 & 0.312958990 \\
\hline $\mathrm{H}$ & 3.357830930 & 4.614541570 & 0.299656020 \\
\hline 0 & 1.302408690 & 6.141535330 & 0.106755900 \\
\hline C & 1.088524230 & 0.482138300 & 0.041878640 \\
\hline C & 2.102821510 & -0.378930650 & -0.063879710 \\
\hline $\mathrm{H}$ & 0.098552030 & 0.060638310 & 0.072449520 \\
\hline
\end{tabular}




\begin{tabular}{|c|c|c|c|}
\hline $\mathrm{H}$ & 3.130315640 & -0.078663440 & -0.124152530 \\
\hline C & 1.835303050 & -1.830894340 & -0.110839660 \\
\hline 0 & 0.757421210 & -2.339328830 & -0.059666910 \\
\hline 0 & 2.960508760 & -2.524494230 & -0.220588160 \\
\hline C & 2.841765410 & -3.933411180 & -0.277336510 \\
\hline $\mathrm{H}$ & 2.254609300 & -4.231069310 & -1.134150190 \\
\hline $\mathrm{H}$ & 3.848687710 & -4.311024390 & -0.364548000 \\
\hline $\mathrm{H}$ & 2.376243810 & -4.311983760 & 0.621250920 \\
\hline $\mathrm{H}$ & 0.443446630 & 6.532693770 & 0.031596920 \\
\hline \multicolumn{4}{|c|}{ C1-C2-C3-C4 angle: 160} \\
\hline C & -0.011228620 & 4.119301300 & -0.127227100 \\
\hline C & -0.051043030 & 2.691995450 & -0.096913670 \\
\hline C & 1.152460560 & 1.921585460 & 0.122610240 \\
\hline C & 2.396972070 & 2.623159090 & 0.318805770 \\
\hline C & 2.429582690 & 4.058953270 & 0.297831210 \\
\hline C & 1.230964770 & 4.785463470 & 0.070722060 \\
\hline $\mathrm{H}$ & -0.910129790 & 4.681338870 & -0.302780100 \\
\hline $\mathrm{H}$ & -0.982003420 & 2.181049330 & -0.250565050 \\
\hline $\mathrm{H}$ & 3.294763130 & 2.078322800 & 0.529587260 \\
\hline $\mathrm{H}$ & 3.344460410 & 4.595272540 & 0.453074230 \\
\hline 0 & 1.327700760 & 6.133892740 & 0.053449430 \\
\hline C & 1.070235130 & 0.476526830 & 0.066886910 \\
\hline C & 2.080155740 & -0.372120080 & -0.127417120 \\
\hline $\mathrm{H}$ & 0.085023140 & 0.049885390 & 0.146987540 \\
\hline $\mathrm{H}$ & 3.096983470 & -0.057275390 & -0.258218740 \\
\hline C & 1.823437420 & -1.826380110 & -0.182388600 \\
\hline 0 & 0.756963060 & -2.346610670 & -0.061774540 \\
\hline 0 & 2.943526630 & -2.505625020 & -0.389050880 \\
\hline C & 2.833679680 & -3.914417660 & -0.465537900 \\
\hline $\mathrm{H}$ & 2.187436300 & -4.201278580 & -1.282579150 \\
\hline $\mathrm{H}$ & 3.834755740 & -4.279608170 & -0.634475520 \\
\hline $\mathrm{H}$ & 2.439335260 & -4.315368890 & 0.457070730 \\
\hline $\mathrm{H}$ & 0.481217230 & 6.530565100 & -0.096859270 \\
\hline \multicolumn{4}{|c|}{ C1-C2-C3-C4 angle: 150} \\
\hline C & 0.015685380 & 4.119716950 & -0.239494100 \\
\hline C & -0.045327670 & 2.693321220 & -0.180770740 \\
\hline C & 1.127210350 & 1.918687750 & 0.153950970 \\
\hline C & 2.363478600 & 2.608588870 & 0.426578600 \\
\hline C & 2.416587330 & 4.042322810 & 0.380590900 \\
\hline C & 1.247473660 & 4.776149900 & 0.044022730 \\
\hline $\mathrm{H}$ & -0.857888720 & 4.688214970 & -0.500977050 \\
\hline $\mathrm{H}$ & -0.966763290 & 2.188592520 & -0.398904750 \\
\hline $\mathrm{H}$ & 3.232234510 & 2.053981300 & 0.719565130 \\
\hline $\mathrm{H}$ & 3.322123710 & 4.572594610 & 0.598570520 \\
\hline
\end{tabular}




\begin{tabular}{|c|c|c|c|}
\hline 0 & 1.361787040 & 6.122634220 & 0.005943340 \\
\hline C & 1.045496740 & 0.469155570 & 0.092090340 \\
\hline C & 2.046084460 & -0.363488320 & -0.188194470 \\
\hline $\mathrm{H}$ & 0.068551400 & 0.035461360 & 0.223474030 \\
\hline $\mathrm{H}$ & 3.047293750 & -0.030688720 & -0.382561660 \\
\hline C & 1.802929900 & -1.820558890 & -0.252962540 \\
\hline 0 & 0.753416910 & -2.355934600 & -0.068754830 \\
\hline 0 & 2.913409420 & -2.481063980 & -0.550385190 \\
\hline C & 2.815044100 & -3.889573530 & -0.647367600 \\
\hline $\mathrm{H}$ & 2.115971230 & -4.169013210 & -1.422391340 \\
\hline $\mathrm{H}$ & 3.805886030 & -4.238110310 & -0.893827520 \\
\hline $\mathrm{H}$ & 2.491753180 & -4.313292570 & 0.292539020 \\
\hline $\mathrm{H}$ & 0.534431170 & 6.524927570 & -0.217999720 \\
\hline \multicolumn{4}{|c|}{ C1-C2-C3-C4 angle: 140} \\
\hline C & 0.050969420 & 4.117863290 & -0.348850950 \\
\hline C & -0.031928250 & 2.692734870 & -0.268954120 \\
\hline C & 1.100233690 & 1.916765670 & 0.177842130 \\
\hline C & 2.322958760 & 2.596382590 & 0.525336050 \\
\hline C & 2.396934890 & 4.027523060 & 0.461397710 \\
\hline C & 1.265113210 & 4.766494210 & 0.020704730 \\
\hline $\mathrm{H}$ & -0.790285840 & 4.690827340 & -0.693307570 \\
\hline $\mathrm{H}$ & -0.938372660 & 2.192802570 & -0.551415220 \\
\hline $\mathrm{H}$ & 3.159532900 & 2.033274740 & 0.888599270 \\
\hline $\mathrm{H}$ & 3.289052850 & 4.552894260 & 0.738461250 \\
\hline 0 & 1.397677430 & 6.110579770 & -0.033170790 \\
\hline C & 1.020319080 & 0.461350860 & 0.114534970 \\
\hline C & 2.004971770 & -0.354267720 & -0.249976400 \\
\hline $\mathrm{H}$ & 0.056418330 & 0.019196960 & 0.304068970 \\
\hline $\mathrm{H}$ & 2.985629440 & -0.003258010 & -0.508622730 \\
\hline C & 1.775468110 & -1.814359180 & -0.320293480 \\
\hline 0 & 0.748345610 & -2.365714180 & -0.070452990 \\
\hline 0 & 2.869493420 & -2.455081860 & -0.707278560 \\
\hline C & 2.781470430 & -3.863123120 & -0.821120920 \\
\hline $\mathrm{H}$ & 2.033396320 & -4.139002320 & -1.550326130 \\
\hline $\mathrm{H}$ & 3.756786150 & -4.194540900 & -1.141852240 \\
\hline $\mathrm{H}$ & 2.529607670 & -4.306085400 & 0.131652370 \\
\hline $\mathrm{H}$ & 0.593675570 & 6.517148560 & -0.324375140 \\
\hline \multicolumn{4}{|c|}{ C1-C2-C3-C4 angle: 130} \\
\hline C & 0.094928980 & 4.115553980 & -0.457057100 \\
\hline C & -0.006992710 & 2.691260440 & -0.367324940 \\
\hline C & 1.076108780 & 1.916478940 & 0.186751470 \\
\hline C & 2.277187080 & 2.588183050 & 0.614159740 \\
\hline C & 2.369773590 & 4.016916130 & 0.541177810 \\
\hline$C$ & 1.281233570 & 4.759088170 & 0.004225160 \\
\hline
\end{tabular}




\begin{tabular}{|c|c|c|c|}
\hline $\mathrm{H}$ & -0.709807580 & 4.691483690 & -0.875746580 \\
\hline $\mathrm{H}$ & -0.891246120 & 2.194222450 & -0.717067510 \\
\hline $\mathrm{H}$ & 3.079932340 & 2.018438130 & 1.038858970 \\
\hline $\mathrm{H}$ & 3.241100000 & 4.539083490 & 0.882824810 \\
\hline 0 & 1.428012430 & 6.101445790 & -0.052295960 \\
\hline C & 0.998291330 & 0.454621750 & 0.127415650 \\
\hline C & 1.958637050 & -0.345532660 & -0.319606430 \\
\hline $\mathrm{H}$ & 0.054056860 & 0.003684200 & 0.385192160 \\
\hline $\mathrm{H}$ & 2.913162800 & 0.021592630 & -0.646150130 \\
\hline C & 1.742090630 & -1.808864700 & -0.385441300 \\
\hline 0 & 0.745001160 & -2.374867990 & -0.059493810 \\
\hline 0 & 2.809282900 & -2.431712200 & -0.864533380 \\
\hline C & 2.730937360 & -3.840118520 & -0.982730510 \\
\hline $\mathrm{H}$ & 1.928239210 & -4.121072820 & -1.649137090 \\
\hline $\mathrm{H}$ & 3.680380820 & -4.155353990 & -1.386524400 \\
\hline $\mathrm{H}$ & 2.565634850 & -4.293342020 & -0.015939160 \\
\hline $\mathrm{H}$ & 0.653226690 & 6.510417230 & -0.411435350 \\
\hline \multicolumn{4}{|c|}{ C1-C2-C3-C4 angle: 120} \\
\hline C & 0.144763320 & 4.116257370 & -0.558159520 \\
\hline C & 0.032250310 & 2.691556060 & -0.475205370 \\
\hline C & 1.059877540 & 1.919515820 & 0.176870860 \\
\hline C & 2.227783980 & 2.586083130 & 0.692792930 \\
\hline C & 2.332612580 & 4.013394040 & 0.623586040 \\
\hline C & 1.292571070 & 4.757281040 & -0.002323270 \\
\hline $\mathrm{H}$ & -0.619187580 & 4.693787280 & -1.045400330 \\
\hline $\mathrm{H}$ & -0.820779820 & 2.195225880 & -0.896058530 \\
\hline $\mathrm{H}$ & 2.996035200 & 2.011092080 & 1.171104560 \\
\hline $\mathrm{H}$ & 3.177893450 & 4.533618290 & 1.027823030 \\
\hline 0 & 1.448717510 & 6.098731320 & -0.052345710 \\
\hline C & 0.983196470 & 0.449277990 & 0.127412330 \\
\hline C & 1.907109790 & -0.339400160 & -0.402355680 \\
\hline $\mathrm{H}$ & 0.071137090 & -0.008937860 & 0.473951850 \\
\hline $\mathrm{H}$ & 2.825171640 & 0.039627970 & -0.810616510 \\
\hline C & 1.703747040 & -1.806614780 & -0.449130230 \\
\hline 0 & 0.744888540 & -2.384027940 & -0.040165350 \\
\hline 0 & 2.734156220 & -2.416589510 & -1.016618290 \\
\hline C & 2.662894630 & -3.825918600 & -1.128978550 \\
\hline $\mathrm{H}$ & 1.811843880 & -4.116225380 & -1.727992680 \\
\hline $\mathrm{H}$ & 3.579844930 & -4.130149460 & -1.609068350 \\
\hline $\mathrm{H}$ & 2.582175390 & -4.280794730 & -0.152287610 \\
\hline $\mathrm{H}$ & 0.706004060 & 6.509043240 & -0.472536470 \\
\hline \multicolumn{4}{|c|}{ C1-C2-C3-C4 angle: 110} \\
\hline C & 0.206094440 & 4.122548380 & -0.661815650 \\
\hline C & 0.102290020 & 2.695221480 & -0.609112400 \\
\hline
\end{tabular}




\begin{tabular}{|c|c|c|c|}
\hline C & 1.073834710 & 1.927577640 & 0.125688480 \\
\hline C & 2.179405120 & 2.594790550 & 0.761079830 \\
\hline C & 2.277848230 & 4.023318750 & 0.718421540 \\
\hline C & 1.291890830 & 4.765931030 & 0.007144670 \\
\hline $\mathrm{H}$ & -0.517145660 & 4.700784350 & -1.206882720 \\
\hline $\mathrm{H}$ & -0.703640110 & 2.195737230 & -1.110943620 \\
\hline $\mathrm{H}$ & 2.907414010 & 2.018176510 & 1.296960790 \\
\hline $\mathrm{H}$ & 3.076599120 & 4.545964040 & 1.205553630 \\
\hline 0 & 1.436675580 & 6.109230180 & -0.009294360 \\
\hline C & 0.991277160 & 0.450421610 & 0.104551540 \\
\hline C & 1.856609190 & -0.339414890 & -0.510950810 \\
\hline $\mathrm{H}$ & 0.132120700 & -0.006736700 & 0.568687910 \\
\hline $\mathrm{H}$ & 2.720279290 & 0.040511400 & -1.024142760 \\
\hline C & 1.664910540 & -1.810034310 & -0.510760520 \\
\hline 0 & 0.763288340 & -2.388023270 & 0.011220300 \\
\hline 0 & 2.633250320 & -2.420567350 & -1.177708040 \\
\hline C & 2.566222820 & -3.832469560 & -1.257019780 \\
\hline $\mathrm{H}$ & 1.657426880 & -4.141808710 & -1.752754350 \\
\hline $\mathrm{H}$ & 3.427802150 & -4.136746310 & -1.830509160 \\
\hline $\mathrm{H}$ & 2.599424880 & -4.269789940 & -0.269603170 \\
\hline $\mathrm{H}$ & 0.733501920 & 6.518055070 & -0.494035710 \\
\hline \multicolumn{4}{|c|}{ C1-C2-C3-C4 angle: 100} \\
\hline C & 0.280415820 & 4.132858460 & -0.762957150 \\
\hline C & 0.203879200 & 2.703127430 & -0.752589340 \\
\hline C & 1.118665070 & 1.939082730 & 0.053645430 \\
\hline C & 2.127399380 & 2.610679990 & 0.828124400 \\
\hline C & 2.201799880 & 4.041671190 & 0.823022540 \\
\hline C & 1.278789330 & 4.781067540 & 0.027905770 \\
\hline $\mathrm{H}$ & -0.399975260 & 4.710406470 & -1.361325390 \\
\hline $\mathrm{H}$ & -0.537370240 & 2.198763360 & -1.341347430 \\
\hline $\mathrm{H}$ & 2.809672900 & 2.035433810 & 1.422572250 \\
\hline $\mathrm{H}$ & 2.934354180 & 4.568500440 & 1.401119960 \\
\hline 0 & 1.394232750 & 6.126784400 & 0.056042460 \\
\hline C & 1.023204130 & 0.458492430 & 0.065908250 \\
\hline C & 1.806849400 & -0.344692550 & -0.633454980 \\
\hline $\mathrm{H}$ & 0.242692080 & 0.012828790 & 0.662012350 \\
\hline $\mathrm{H}$ & 2.596291710 & 0.024038770 & -1.261624790 \\
\hline C & 1.625044690 & -1.816085800 & -0.572751230 \\
\hline 0 & 0.799261420 & -2.382582420 & 0.072340410 \\
\hline 0 & 2.507165080 & -2.440639810 & -1.338642270 \\
\hline C & 2.441374730 & -3.854531780 & -1.371375840 \\
\hline $\mathrm{H}$ & 1.480539580 & -4.180983090 & -1.742273690 \\
\hline $\mathrm{H}$ & 3.227631630 & -4.170811560 & -2.038839070 \\
\hline $\mathrm{H}$ & 2.599748980 & -4.264428170 & -0.384349100 \\
\hline
\end{tabular}




$\begin{array}{lrrr}\text { H } & 0.739386410 & 6.532724180 & -0.494388560 \\ \text { C1-C2-C3-C4 angle: } 90 & & \\ \text { C } & 0.367056480 & 4.140352250 & -0.857421910 \\ \mathrm{C} & 0.321309720 & 2.710253520 & -0.878715030 \\ \mathrm{C} & 1.173390410 & 1.948804960 & -0.003764820 \\ \mathrm{C} & 2.062889200 & 2.625466760 & 0.901051480 \\ \mathrm{C} & 2.110833560 & 4.058302900 & 0.922595880 \\ \mathrm{C} & 1.263654250 & 4.793543900 & 0.043748880 \\ \mathrm{H} & -0.264780230 & 4.715437900 & -1.509063850 \\ \mathrm{H} & -0.347134580 & 2.201306090 & -1.545277480 \\ \mathrm{H} & 2.690316270 & 2.053825280 & 1.556508360 \\ \mathrm{H} & 2.766201900 & 4.588918910 & 1.583882850 \\ \mathrm{O} & 1.347232070 & 6.140503370 & 0.104798700 \\ \mathrm{C} & 1.063789030 & 0.468764050 & 0.032372370 \\ \mathrm{C} & 1.755741180 & -0.349280980 & -0.741933950 \\ \mathrm{H} & 0.377564360 & 0.036713620 & 0.743599210 \\ \mathrm{H} & 2.460894600 & 0.005792880 & -1.470259750 \\ \mathrm{C} & 1.582417720 & -1.819011940 & -0.632199150 \\ \mathrm{O} & 0.841958070 & -2.371440220 & 0.119733990 \\ \mathrm{O} & 2.365371060 & -2.459763510 & -1.487308950 \\ \mathrm{C} & 2.297998400 & -3.873981210 & -1.483369000 \\ \mathrm{H} & 1.299565020 & -4.206750220 & -1.727784250 \\ \mathrm{H} & 2.997510840 & -4.204503840 & -2.235162790 \\ \mathrm{H} & 2.576010750 & -4.264105690 & -0.514955670 \\ \mathrm{H} & 0.750320410 & 6.543317940 & -0.510037880 \\ \mathrm{OMp} & & & \end{array}$

OMpCA (W1)

\section{C1-C2-C3-C4 angle: 180}

$\begin{array}{lrrr}\text { C } & -2.746195880 & 0.798137290 & -0.000529000 \\ \text { C } & -1.346007300 & 1.087616070 & 0.016777370 \\ \text { C } & -0.355197970 & 0.028647580 & -0.004379860 \\ \text { C } & -0.830420410 & -1.335237970 & -0.043705990 \\ \text { C } & -2.242546290 & -1.616408530 & -0.060878680 \\ \text { C } & -3.182296130 & -0.554282820 & -0.039284270 \\ \text { H } & -3.463459260 & 1.596429270 & 0.015625320 \\ \text { H } & -1.016320690 & 2.108601430 & 0.046036770 \\ \text { H } & -0.136167870 & -2.150418880 & -0.060470750 \\ \text { H } & -2.598639200 & -2.627256560 & -0.090056420 \\ \text { O } & -4.487668930 & -0.886151050 & -0.057060210 \\ \text { C } & 1.030145960 & 0.379839330 & 0.014453300 \\ \text { C } & 2.082841510 & -0.447460010 & -0.000154280 \\ \text { H } & 1.252035790 & 1.432979940 & 0.043816500 \\ \text { H } & 1.996440030 & -1.515657740 & -0.028980890 \\ \text { C } & 3.451529480 & 0.101173610 & 0.023779400 \\ \text { O } & 3.738331570 & 1.259022420 & 0.056353770\end{array}$




\begin{tabular}{|c|c|c|c|}
\hline 0 & 4.359840610 & -0.867406830 & 0.004444300 \\
\hline C & 5.717231310 & -0.471621070 & 0.024258720 \\
\hline $\mathrm{H}$ & 5.937530170 & 0.088925790 & 0.921455670 \\
\hline $\mathrm{H}$ & 6.291673040 & -1.384923030 & 0.004082030 \\
\hline $\mathrm{H}$ & 5.949662710 & 0.135448020 & -0.838961690 \\
\hline $\mathrm{H}$ & -5.043323500 & -0.112014330 & -0.040436390 \\
\hline 0 & -6.295203170 & 1.382983500 & -0.009570540 \\
\hline $\mathrm{H}$ & -6.864253030 & 1.438763660 & 0.747010040 \\
\hline $\mathrm{H}$ & -6.853776150 & 1.478855310 & -0.769931080 \\
\hline \multicolumn{4}{|c|}{ C1-C2-C3-C4 angle: 170} \\
\hline C & -2.747416740 & 0.784957460 & 0.088325550 \\
\hline C & -1.369494770 & 1.115866480 & 0.058631740 \\
\hline C & -0.343111110 & 0.078232890 & -0.077457450 \\
\hline C & -0.810068800 & -1.295395960 & -0.171471880 \\
\hline C & -2.230172740 & -1.577054780 & -0.140714200 \\
\hline C & -3.159389050 & -0.526526270 & -0.009479700 \\
\hline $\mathrm{H}$ & -3.478347060 & 1.565849660 & 0.192594710 \\
\hline $\mathrm{H}$ & -1.061960430 & 2.140355100 & 0.140081720 \\
\hline $\mathrm{H}$ & -0.119451820 & -2.102688590 & -0.301151550 \\
\hline $\mathrm{H}$ & -2.591271800 & -2.583648430 & -0.213856730 \\
\hline 0 & -4.450936990 & -0.882975470 & 0.013924460 \\
\hline C & 1.011390790 & 0.440933860 & -0.047203960 \\
\hline C & 2.088159090 & -0.426537460 & 0.032414920 \\
\hline $\mathrm{H}$ & 1.243152720 & 1.490797060 & -0.026412370 \\
\hline $\mathrm{H}$ & 1.978325480 & -1.491712060 & 0.053236560 \\
\hline C & 3.464797510 & 0.095210850 & 0.094269750 \\
\hline 0 & 3.767765490 & 1.248076970 & 0.076410530 \\
\hline 0 & 4.351561440 & -0.888618610 & 0.176171620 \\
\hline C & 5.715054250 & -0.516601970 & 0.244875240 \\
\hline $\mathrm{H}$ & 5.899806560 & 0.090340500 & 1.119492270 \\
\hline $\mathrm{H}$ & 6.269905390 & -1.439860430 & 0.306346210 \\
\hline $\mathrm{H}$ & 6.003323960 & 0.035531700 & -0.638038030 \\
\hline $\mathrm{H}$ & -5.021826850 & -0.123443700 & 0.100827230 \\
\hline 0 & -6.318106520 & 1.307152650 & 0.267638330 \\
\hline $\mathrm{H}$ & -6.861571180 & 1.313198460 & 1.044719260 \\
\hline $\mathrm{H}$ & -6.900607760 & 1.451705230 & -0.466554150 \\
\hline \multicolumn{4}{|c|}{ C1-C2-C3-C4 angle: 160} \\
\hline C & -2.741775190 & 0.785752470 & 0.193122400 \\
\hline C & -1.344321220 & 1.092937720 & 0.142477820 \\
\hline C & -0.357831760 & 0.058615050 & -0.096024950 \\
\hline C & -0.823625950 & -1.295042690 & -0.291432470 \\
\hline C & -2.232008720 & -1.592901490 & -0.250251290 \\
\hline C & -3.171862580 & -0.556967420 & -0.004025350 \\
\hline $\mathrm{H}$ & -3.460061960 & 1.560145130 & 0.383450360 \\
\hline
\end{tabular}




\begin{tabular}{|c|c|c|c|}
\hline $\mathrm{H}$ & -1.017137640 & 2.104011370 & 0.293182260 \\
\hline $\mathrm{H}$ & -0.126748070 & -2.076779270 & -0.517488410 \\
\hline $\mathrm{H}$ & -2.586558070 & -2.592692630 & -0.405309440 \\
\hline 0 & -4.473184950 & -0.903037300 & 0.032150170 \\
\hline C & 1.039776100 & 0.407629820 & -0.050575190 \\
\hline C & 2.069362960 & -0.419613380 & 0.125393520 \\
\hline $\mathrm{H}$ & 1.270516090 & 1.457629400 & -0.115738940 \\
\hline $\mathrm{H}$ & 1.959466960 & -1.479987390 & 0.243055180 \\
\hline C & 3.446831530 & 0.112816790 & 0.173818640 \\
\hline 0 & 3.753867380 & 1.259959840 & 0.063969080 \\
\hline 0 & 4.331597500 & -0.858017340 & 0.362095580 \\
\hline C & 5.692734820 & -0.479335720 & 0.430708880 \\
\hline $\mathrm{H}$ & 5.857464550 & 0.200714290 & 1.254106660 \\
\hline $\mathrm{H}$ & 6.246036770 & -1.392917720 & 0.584179900 \\
\hline $\mathrm{H}$ & 6.002288500 & -0.004317210 & -0.489141510 \\
\hline $\mathrm{H}$ & -5.029378120 & -0.146897890 & 0.197047910 \\
\hline 0 & -6.289662290 & 1.304685650 & 0.530438750 \\
\hline $\mathrm{H}$ & -6.803373450 & 1.234827260 & 1.324516620 \\
\hline $\mathrm{H}$ & -6.903426590 & 1.493940110 & -0.167277220 \\
\hline \multicolumn{4}{|c|}{ C1-C2-C3-C4 angle: 150} \\
\hline C & -2.741486900 & 0.722059920 & 0.443378740 \\
\hline C & -1.377247850 & 1.112508540 & 0.361688480 \\
\hline C & -0.349170020 & 0.164700870 & -0.070588600 \\
\hline C & -0.788483140 & -1.190044040 & -0.376682900 \\
\hline C & -2.190761100 & -1.528411230 & -0.290974470 \\
\hline C & -3.126479720 & -0.565715450 & 0.121299250 \\
\hline $\mathrm{H}$ & -3.478748690 & 1.432450030 & 0.769186240 \\
\hline $\mathrm{H}$ & -1.087627810 & 2.111026720 & 0.625808860 \\
\hline $\mathrm{H}$ & -0.091353370 & -1.913420550 & -0.748174480 \\
\hline $\mathrm{H}$ & -2.533068070 & -2.515730070 & -0.530133120 \\
\hline 0 & -4.402553010 & -0.968639620 & 0.184305480 \\
\hline C & 1.012835750 & 0.526767550 & 0.018857740 \\
\hline C & 2.064675800 & -0.338997330 & 0.223395790 \\
\hline $\mathrm{H}$ & 1.239403960 & 1.576200760 & 0.093412940 \\
\hline $\mathrm{H}$ & 1.941297390 & -1.403088070 & 0.254727860 \\
\hline C & 3.434958300 & 0.177399690 & 0.409206130 \\
\hline 0 & 3.749265340 & 1.326364830 & 0.380501180 \\
\hline 0 & 4.296494110 & -0.807965770 & 0.622686170 \\
\hline C & 5.648871940 & -0.443179310 & 0.825200490 \\
\hline $\mathrm{H}$ & 5.744280570 & 0.195491400 & 1.691437300 \\
\hline $\mathrm{H}$ & 6.183291630 & -1.367295880 & 0.981035010 \\
\hline $\mathrm{H}$ & 6.036918870 & 0.072632080 & -0.041248860 \\
\hline $\mathrm{H}$ & -4.978434190 & -0.264064490 & 0.470709710 \\
\hline 0 & -6.288502520 & 1.039967410 & 1.057578090 \\
\hline
\end{tabular}




\begin{tabular}{|c|c|c|c|}
\hline $\mathrm{H}$ & -6.733267930 & 0.858092600 & 1.875089630 \\
\hline $\mathrm{H}$ & -6.961443500 & 1.288255380 & 0.437325760 \\
\hline \multicolumn{4}{|c|}{ C1-C2-C3-C4 angle: 140} \\
\hline C & -2.708629420 & 0.735215610 & 0.355077120 \\
\hline C & -1.347923610 & 1.120836320 & 0.194021640 \\
\hline C & -0.350317220 & 0.163825370 & -0.284001850 \\
\hline C & -0.808639510 & -1.198927270 & -0.530635180 \\
\hline C & -2.204325260 & -1.529999100 & -0.369755780 \\
\hline C & -3.113215770 & -0.558367670 & 0.072798730 \\
\hline $\mathrm{H}$ & -3.425026910 & 1.451127500 & 0.713406130 \\
\hline $\mathrm{H}$ & -1.041824250 & 2.121297350 & 0.430797970 \\
\hline $\mathrm{H}$ & -0.129321570 & -1.930998820 & -0.918836000 \\
\hline $\mathrm{H}$ & -2.561409230 & -2.520030550 & -0.573861730 \\
\hline 0 & -4.386203170 & -0.952358770 & 0.208729940 \\
\hline C & 1.026746730 & 0.498625140 & -0.218697550 \\
\hline C & 2.042895760 & -0.356770910 & 0.131017710 \\
\hline $\mathrm{H}$ & 1.276734270 & 1.545681930 & -0.243687860 \\
\hline $\mathrm{H}$ & 1.887470280 & -1.406365420 & 0.284134820 \\
\hline C & 3.422282570 & 0.145407480 & 0.298692360 \\
\hline 0 & 3.771485920 & 1.272177360 & 0.133175200 \\
\hline 0 & 4.245330780 & -0.824760430 & 0.671631740 \\
\hline C & 5.600298570 & -0.471217120 & 0.877587340 \\
\hline $\mathrm{H}$ & 5.684434600 & 0.272037000 & 1.657266790 \\
\hline $\mathrm{H}$ & 6.100754770 & -1.380535890 & 1.171860420 \\
\hline $\mathrm{H}$ & 6.033720680 & -0.082454550 & -0.032572500 \\
\hline $\mathrm{H}$ & -4.945258340 & -0.236487870 & 0.500295240 \\
\hline 0 & -6.231109850 & 1.100063740 & 1.067825640 \\
\hline $\mathrm{H}$ & -6.703534170 & 0.930165020 & 1.872296050 \\
\hline $\mathrm{H}$ & -6.879553790 & 1.365071400 & 0.428620560 \\
\hline \multicolumn{4}{|c|}{ C1-C2-C3-C4 angle: 130} \\
\hline C & -2.688971780 & 0.709873910 & 0.437433060 \\
\hline C & -1.336696850 & 1.120748660 & 0.243638020 \\
\hline C & -0.358103920 & 0.206067170 & -0.341263400 \\
\hline C & -0.812683700 & -1.149350410 & -0.639396000 \\
\hline C & -2.195730840 & -1.503846110 & -0.445880520 \\
\hline C & -3.092067810 & -0.572620730 & 0.090063990 \\
\hline $\mathrm{H}$ & -3.396317800 & 1.391890620 & 0.871205300 \\
\hline $\mathrm{H}$ & -1.029097990 & 2.107581540 & 0.530486120 \\
\hline $\mathrm{H}$ & -0.138478330 & -1.849834590 & -1.090720380 \\
\hline $\mathrm{H}$ & -2.553113230 & -2.483539060 & -0.694899140 \\
\hline 0 & -4.355351790 & -0.984016770 & 0.253826360 \\
\hline C & 1.033983280 & 0.525273730 & -0.252013020 \\
\hline C & 2.016500570 & -0.315945780 & 0.183607590 \\
\hline $\mathrm{H}$ & 1.298706080 & 1.567318790 & -0.323171870 \\
\hline
\end{tabular}




\begin{tabular}{|c|c|c|c|}
\hline $\mathrm{H}$ & 1.838490070 & -1.353455240 & 0.388866820 \\
\hline C & 3.400897260 & 0.175903030 & 0.367551840 \\
\hline 0 & 3.775595730 & 1.283128820 & 0.141368070 \\
\hline 0 & 4.191069910 & -0.779919330 & 0.833883930 \\
\hline C & 5.544339020 & -0.435914230 & 1.067474770 \\
\hline $\mathrm{H}$ & 5.613545520 & 0.355717820 & 1.799489240 \\
\hline $\mathrm{H}$ & 6.016206380 & -1.331953880 & 1.439252720 \\
\hline $\mathrm{H}$ & 6.017820770 & -0.115324000 & 0.150777040 \\
\hline $\mathrm{H}$ & -4.906653580 & -0.291675770 & 0.609936470 \\
\hline 0 & -6.182150000 & 0.986689020 & 1.315759460 \\
\hline $\mathrm{H}$ & -6.614627410 & 0.756108790 & 2.127564420 \\
\hline $\mathrm{H}$ & -6.863443710 & 1.278459980 & 0.724187530 \\
\hline \multicolumn{4}{|c|}{ C1-C2-C3-C4 angle: 120} \\
\hline C & -2.669271600 & 0.672160760 & 0.541008920 \\
\hline C & -1.325513470 & 1.110916980 & 0.326125030 \\
\hline C & -0.369794330 & 0.250159340 & -0.367049920 \\
\hline C & -0.819451870 & -1.092350370 & -0.728124990 \\
\hline C & -2.190491360 & -1.472588240 & -0.514154530 \\
\hline C & -3.072986130 & -0.590479970 & 0.115833680 \\
\hline $\mathrm{H}$ & -3.366052970 & 1.311785700 & 1.049586310 \\
\hline $\mathrm{H}$ & -1.015285920 & 2.078723750 & 0.669412450 \\
\hline $\mathrm{H}$ & -0.151517030 & -1.755339910 & -1.241778760 \\
\hline $\mathrm{H}$ & -2.549982830 & -2.436120740 & -0.817552600 \\
\hline 0 & -4.326387050 & -1.022063050 & 0.295479810 \\
\hline C & 1.040446280 & 0.556422730 & -0.259054840 \\
\hline C & 1.988794930 & -0.263563050 & 0.253415100 \\
\hline $\mathrm{H}$ & 1.324909960 & 1.586775090 & -0.400179810 \\
\hline $\mathrm{H}$ & 1.786337860 & -1.282868530 & 0.520517770 \\
\hline C & 3.383777530 & 0.214071220 & 0.432081540 \\
\hline 0 & 3.786133320 & 1.293340300 & 0.134523580 \\
\hline 0 & 4.140927510 & -0.720661950 & 0.984341410 \\
\hline C & 5.496894790 & -0.389795660 & 1.224483090 \\
\hline $\mathrm{H}$ & 5.567771330 & 0.451176650 & 1.898965140 \\
\hline $\mathrm{H}$ & 5.939351760 & -1.266520140 & 1.670733310 \\
\hline $\mathrm{H}$ & 5.997115640 & -0.146857080 & 0.298247040 \\
\hline $\mathrm{H}$ & -4.869839140 & -0.359166520 & 0.714287660 \\
\hline 0 & -6.141078960 & 0.857614130 & 1.527856710 \\
\hline $\mathrm{H}$ & -6.575159730 & 0.560444620 & 2.316877520 \\
\hline $\mathrm{H}$ & -6.821619080 & 1.194755440 & 0.959953100 \\
\hline \multicolumn{4}{|c|}{ C1-C2-C3-C4 angle: 110} \\
\hline C & -2.711354980 & 0.548529900 & 0.792683510 \\
\hline C & -1.321459570 & 0.903985740 & 0.793528570 \\
\hline C & -0.378082030 & 0.078957160 & 0.056761100 \\
\hline C & -0.862884250 & -1.089835950 & -0.653034260 \\
\hline
\end{tabular}




\begin{tabular}{|c|c|c|c|}
\hline C & -2.273532090 & -1.381823410 & -0.655064060 \\
\hline C & -3.147717150 & -0.563737310 & 0.071312490 \\
\hline $\mathrm{H}$ & -3.422988470 & 1.143447850 & 1.333542630 \\
\hline $\mathrm{H}$ & -0.979929490 & 1.772079790 & 1.322119650 \\
\hline $\mathrm{H}$ & -0.177529280 & -1.704911080 & -1.201934900 \\
\hline $\mathrm{H}$ & -2.669643770 & -2.218076970 & -1.195517840 \\
\hline 0 & -4.438032220 & -0.901852340 & 0.039515360 \\
\hline C & 1.053820470 & 0.462988640 & -0.008363700 \\
\hline C & 2.036822810 & -0.201932700 & 0.606914040 \\
\hline $\mathrm{H}$ & 1.315374090 & 1.346153640 & -0.569624260 \\
\hline $\mathrm{H}$ & 1.853589680 & -1.084173020 & 1.191421690 \\
\hline C & 3.451248920 & 0.255942540 & 0.500708250 \\
\hline 0 & 3.818200050 & 1.216456360 & -0.096610950 \\
\hline 0 & 4.264335540 & -0.550373610 & 1.162587390 \\
\hline C & 5.644236880 & -0.229741140 & 1.143012490 \\
\hline $\mathrm{H}$ & 5.812320660 & 0.744307090 & 1.578974370 \\
\hline $\mathrm{H}$ & 6.131966020 & -0.992437360 & 1.729328810 \\
\hline $\mathrm{H}$ & 6.019757510 & -0.236871940 & 0.130091600 \\
\hline $\mathrm{H}$ & -4.973618220 & -0.299477540 & 0.550474660 \\
\hline 0 & -6.257410970 & 0.785266910 & 1.512828390 \\
\hline $\mathrm{H}$ & -6.735570740 & 0.356968400 & 2.210772540 \\
\hline $\mathrm{H}$ & -6.902694470 & 1.239909550 & 0.987348190 \\
\hline \multicolumn{4}{|c|}{ C1-C2-C3-C4 angle: 100} \\
\hline C & -2.697035950 & 0.437806370 & 0.918711970 \\
\hline C & -1.299668770 & 0.723015910 & 0.991650640 \\
\hline C & -0.377192640 & -0.010611740 & 0.139391610 \\
\hline C & -0.890179290 & -1.077919220 & -0.702465530 \\
\hline C & -2.303545320 & -1.329719980 & -0.739116080 \\
\hline C & -3.160169720 & -0.567923670 & 0.068488180 \\
\hline $\mathrm{H}$ & -3.397619300 & 0.997707970 & 1.509178630 \\
\hline $\mathrm{H}$ & -0.931839790 & 1.504421900 & 1.626955850 \\
\hline $\mathrm{H}$ & -0.218009700 & -1.631821170 & -1.327438880 \\
\hline $\mathrm{H}$ & -2.722468120 & -2.081871870 & -1.377169300 \\
\hline 0 & -4.458448810 & -0.851933290 & -0.017975830 \\
\hline C & 1.039058990 & 0.408062530 & 0.038543720 \\
\hline C & 2.043037210 & -0.182469230 & 0.704872900 \\
\hline $\mathrm{H}$ & 1.273488460 & 1.246369150 & -0.598111990 \\
\hline $\mathrm{H}$ & 1.881542400 & -1.017287660 & 1.360911450 \\
\hline C & 3.440206130 & 0.298730800 & 0.558005130 \\
\hline 0 & 3.786199080 & 1.211424390 & -0.123526150 \\
\hline 0 & 4.275720450 & -0.422201900 & 1.290935160 \\
\hline C & 5.644857570 & -0.065769720 & 1.240251260 \\
\hline $\mathrm{H}$ & 5.785872600 & 0.948213390 & 1.585700020 \\
\hline $\mathrm{H}$ & 6.154044130 & -0.757838440 & 1.892618100 \\
\hline
\end{tabular}




\begin{tabular}{|c|c|c|c|}
\hline $\mathrm{H}$ & 6.022648100 & -0.153817400 & 0.231845550 \\
\hline $\mathrm{H}$ & -4.982362820 & -0.298762360 & 0.557430070 \\
\hline 0 & -6.228196310 & 0.700807960 & 1.639946670 \\
\hline $\mathrm{H}$ & -6.702087940 & 0.221047000 & 2.306674060 \\
\hline $\mathrm{H}$ & -6.870303100 & 1.228584340 & 1.183396730 \\
\hline \multicolumn{4}{|c|}{ C1-C2-C3-C4 angle: 90} \\
\hline C & -2.674618700 & 0.318148320 & 1.025111990 \\
\hline C & -1.272172410 & 0.560604280 & 1.111517860 \\
\hline C & -0.372234460 & -0.085997720 & 0.166106920 \\
\hline C & -0.916296170 & -1.036105100 & -0.786507240 \\
\hline C & -2.335109750 & -1.256794120 & -0.827489960 \\
\hline C & -3.167482830 & -0.574735110 & 0.072586860 \\
\hline $\mathrm{H}$ & -3.356053920 & 0.822616180 & 1.683947130 \\
\hline $\mathrm{H}$ & -0.878110240 & 1.253484720 & 1.828477960 \\
\hline $\mathrm{H}$ & -0.265113470 & -1.523638400 & -1.484830130 \\
\hline $\mathrm{H}$ & -2.778351850 & -1.919900610 & -1.543236460 \\
\hline 0 & -4.471700150 & -0.821965920 & -0.030310250 \\
\hline C & 1.034451220 & 0.359586230 & 0.056015590 \\
\hline C & 2.040768430 & -0.150492650 & 0.783565230 \\
\hline $\mathrm{H}$ & 1.262080510 & 1.137409380 & -0.655468360 \\
\hline $\mathrm{H}$ & 1.886560190 & -0.929386130 & 1.506705440 \\
\hline C & 3.428670040 & 0.348421570 & 0.616500970 \\
\hline 0 & 3.767100390 & 1.203812290 & -0.139412350 \\
\hline 0 & 4.266967430 & -0.283248060 & 1.425251260 \\
\hline C & 5.628382050 & 0.099693770 & 1.363705850 \\
\hline $\mathrm{H}$ & 5.740797250 & 1.143663870 & 1.618488430 \\
\hline $\mathrm{H}$ & 6.141524530 & -0.518701940 & 2.083542510 \\
\hline $\mathrm{H}$ & 6.025689080 & -0.069418620 & 0.373290980 \\
\hline $\mathrm{H}$ & -4.979151010 & -0.325387960 & 0.608147230 \\
\hline 0 & -6.193133860 & 0.582588180 & 1.800161340 \\
\hline $\mathrm{H}$ & -6.689300250 & 0.048015800 & 2.406236880 \\
\hline $\mathrm{H}$ & -6.810847570 & 1.186601690 & 1.409138230 \\
\hline \multicolumn{4}{|c|}{ OMpCA (W2) } \\
\hline \multicolumn{4}{|c|}{ C1-C2-C3-C4 angle: 180} \\
\hline C & -2.915762710 & 0.844238000 & -0.027695870 \\
\hline C & -1.499302300 & 1.022895040 & -0.025176080 \\
\hline C & -0.606009310 & -0.115031840 & 0.021050100 \\
\hline C & -1.178860310 & -1.437861090 & 0.065866690 \\
\hline C & -2.605941240 & -1.609249360 & 0.064469920 \\
\hline C & -3.452899600 & -0.471879280 & 0.017590200 \\
\hline $\mathrm{H}$ & -3.566244740 & 1.698730050 & -0.065555340 \\
\hline $\mathrm{H}$ & -1.082389210 & 2.010781360 & -0.067018180 \\
\hline $\mathrm{H}$ & -0.548371940 & -2.302580210 & 0.101411430 \\
\hline $\mathrm{H}$ & -3.044764540 & -2.586606570 & 0.097602400 \\
\hline
\end{tabular}




\begin{tabular}{lrrr} 
O & -4.785902960 & -0.701338200 & 0.018771710 \\
C & 0.813995710 & 0.136246300 & 0.022783960 \\
C & 1.795099080 & -0.772237720 & 0.061667780 \\
H & 1.098491820 & 1.172445470 & -0.008576020 \\
H & 1.622538650 & -1.829722170 & 0.095594990 \\
C & 3.203919760 & -0.345687890 & 0.054541480 \\
O & 3.599366540 & 0.785926390 & 0.016013700 \\
O & 4.024627750 & -1.381445040 & 0.097794220 \\
C & 5.414478640 & -1.110189130 & 0.090163640 \\
H & 5.690572670 & -0.520260610 & 0.952127230 \\
H & 5.901181010 & -2.072203950 & 0.123831040 \\
H & 5.692649790 & -0.580397690 & -0.809218090 \\
O & 2.179018300 & 3.390343480 & -0.120550960 \\
H & 2.764093230 & 2.644335610 & -0.212582540 \\
H & 2.397371460 & 3.769406290 & 0.719661510 \\
H & -5.267298680 & 0.113010800 & -0.020683380 \\
C1-C2-C3-C4 angle: 170 & & \\
C & -2.916640080 & 0.840772410 & 0.081177760 \\
C & -1.501617720 & 1.023940530 & 0.028870530 \\
C & -0.607882520 & -0.111407600 & -0.049587930 \\
C & -1.177413510 & -1.436026500 & -0.080251630 \\
C & -2.602916010 & -1.612075770 & -0.032085450 \\
C & -3.450845080 & -0.477222140 & 0.051143790 \\
H & -3.568314410 & 1.692997760 & 0.142630730 \\
H & -1.087132140 & 2.013603680 & 0.041947620 \\
H & -0.545762510 & -2.296346670 & -0.167703500 \\
H & -3.040208340 & -2.590293260 & -0.059484350 \\
O & -4.782220670 & -0.710729450 & 0.098643490 \\
C & 0.813243210 & 0.140473990 & -0.053664020 \\
C & 1.790442020 & -0.764379240 & 0.066171580 \\
H & 1.099003890 & 1.173848540 & -0.133262310 \\
H & 1.612242070 & -1.816010450 & 0.174093530 \\
C & 3.201163160 & -0.343337770 & 0.056557070 \\
O & 3.601907120 & 0.782291570 & -0.045748380 \\
O & -16461310 & -1.376828670 & 0.180262420 \\
H & -1.110827690 & 0.182586300 \\
H & -0.468939940 & 1.011143520 \\
H & -2.070314390 & 0.285821690 \\
H & -0.639217810 & -0.742628660 \\
H & 3.390151790 & -0.222863460 \\
H & 2.640700950 & -0.306352790 \\
\hline
\end{tabular}

C1-C2-C3-C4 angle: 160 


\begin{tabular}{|c|c|c|c|}
\hline C & -2.916488850 & 0.827876570 & 0.166565070 \\
\hline C & -1.505286550 & 1.023616020 & 0.067601900 \\
\hline C & -0.612165620 & -0.101102200 & -0.101789250 \\
\hline C & -1.174116900 & -1.427105770 & -0.175568700 \\
\hline C & -2.595354250 & -1.615761090 & -0.085092170 \\
\hline C & -3.444480780 & -0.491440360 & 0.089777380 \\
\hline $\mathrm{H}$ & -3.569936270 & 1.670561360 & 0.299213240 \\
\hline $\mathrm{H}$ & -1.095322340 & 2.014128190 & 0.113751180 \\
\hline $\mathrm{H}$ & -0.539713690 & -2.273399280 & -0.345212680 \\
\hline $\mathrm{H}$ & -3.029500250 & -2.593644490 & -0.149134220 \\
\hline 0 & -4.771671570 & -0.735885550 & 0.177547760 \\
\hline C & 0.812168900 & 0.152100110 & -0.108646740 \\
\hline C & 1.779064140 & -0.740739580 & 0.120165280 \\
\hline $\mathrm{H}$ & 1.101605910 & 1.176490180 & -0.261439460 \\
\hline $\mathrm{H}$ & 1.587066870 & -1.776249340 & 0.321992720 \\
\hline C & 3.193954800 & -0.331327210 & 0.109479310 \\
\hline 0 & 3.606654920 & 0.778383530 & -0.079469140 \\
\hline 0 & 3.996373330 & -1.355496020 & 0.343455360 \\
\hline C & 5.389199810 & -1.099648000 & 0.359665260 \\
\hline $\mathrm{H}$ & 5.635415650 & -0.389877420 & 1.136066630 \\
\hline $\mathrm{H}$ & 5.859526850 & -2.049727750 & 0.558570460 \\
\hline $\mathrm{H}$ & 5.714690230 & -0.712317200 & -0.594777870 \\
\hline 0 & 2.226740530 & 3.384239900 & -0.404282330 \\
\hline $\mathrm{H}$ & 2.803334280 & 2.627231780 & -0.443729870 \\
\hline $\mathrm{H}$ & 2.434851370 & 3.806859110 & 0.417475220 \\
\hline $\mathrm{H}$ & -5.256592920 & 0.071321170 & 0.276325540 \\
\hline \multicolumn{4}{|c|}{ C1-C2-C3-C4 angle: 150} \\
\hline C & -2.915074730 & 0.804989290 & 0.263645110 \\
\hline C & -1.509165090 & 1.019252360 & 0.125756740 \\
\hline C & -0.618900810 & -0.086236350 & -0.144940290 \\
\hline C & -1.171577200 & -1.412007170 & -0.270675160 \\
\hline C & -2.586685070 & -1.619070650 & -0.145217900 \\
\hline C & -3.436188480 & -0.513067110 & 0.125573560 \\
\hline $\mathrm{H}$ & -3.569487060 & 1.631370800 & 0.472206620 \\
\hline $\mathrm{H}$ & -1.104506530 & 2.008891440 & 0.215597320 \\
\hline $\mathrm{H}$ & -0.533580830 & -2.238036890 & -0.513359010 \\
\hline $\mathrm{H}$ & -3.017128740 & -2.594646440 & -0.253655420 \\
\hline 0 & -4.757684370 & -0.773346790 & 0.244934930 \\
\hline C & 0.810364580 & 0.167995270 & -0.153999090 \\
\hline C & 1.762544960 & -0.706916700 & 0.173480380 \\
\hline $\mathrm{H}$ & 1.105764140 & 1.178665650 & -0.374756390 \\
\hline $\mathrm{H}$ & 1.551962580 & -1.720181820 & 0.455544900 \\
\hline C & 3.182918810 & -0.312988180 & 0.165198330 \\
\hline 0 & 3.612531150 & 0.773433790 & -0.102678230 \\
\hline
\end{tabular}




\begin{tabular}{|c|c|c|c|}
\hline 0 & 3.966746520 & -1.322248880 & 0.502386350 \\
\hline C & 5.361845100 & -1.079765540 & 0.534138390 \\
\hline $\mathrm{H}$ & 5.596805910 & -0.311837880 & 1.256810930 \\
\hline $\mathrm{H}$ & 5.815504100 & -2.015206790 & 0.821365200 \\
\hline $\mathrm{H}$ & 5.715700030 & -0.774356040 & -0.439721900 \\
\hline 0 & 2.273888800 & 3.368835160 & -0.610904790 \\
\hline $\mathrm{H}$ & 2.839804110 & 2.603131570 & -0.593585060 \\
\hline $\mathrm{H}$ & 2.481307200 & 3.843919520 & 0.181819250 \\
\hline $\mathrm{H}$ & -5.244262240 & 0.022795220 & 0.405872710 \\
\hline \multicolumn{4}{|c|}{ C1-C2-C3-C4 angle: 140} \\
\hline C & -2.913220240 & 0.769455890 & 0.380460100 \\
\hline C & -1.513187600 & 1.007489490 & 0.215884640 \\
\hline C & -0.628294210 & -0.065601830 & -0.171027390 \\
\hline C & -1.171757680 & -1.385908270 & -0.369943480 \\
\hline C & -2.580011660 & -1.616195860 & -0.221471180 \\
\hline C & -3.428549710 & -0.540221750 & 0.156944180 \\
\hline $\mathrm{H}$ & -3.567000570 & 1.569762390 & 0.674877640 \\
\hline $\mathrm{H}$ & -1.113293270 & 1.991092120 & 0.369644510 \\
\hline $\mathrm{H}$ & -0.530308550 & -2.183758430 & -0.687610610 \\
\hline $\mathrm{H}$ & -3.007015530 & -2.584813210 & -0.389252800 \\
\hline 0 & -4.743683790 & -0.820671780 & 0.296281030 \\
\hline C & 0.807168180 & 0.189647470 & -0.180976380 \\
\hline C & 1.742553540 & -0.664750410 & 0.229794760 \\
\hline $\mathrm{H}$ & 1.110404080 & 1.183445770 & -0.462334530 \\
\hline $\mathrm{H}$ & 1.511803580 & -1.653448680 & 0.577125200 \\
\hline C & 3.168760080 & -0.287507260 & 0.227159940 \\
\hline 0 & 3.617999640 & 0.770680170 & -0.110598730 \\
\hline 0 & 3.930611910 & -1.276806530 & 0.659163480 \\
\hline C & 5.327697480 & -1.048125950 & 0.708711170 \\
\hline $\mathrm{H}$ & 5.554321500 & -0.230367870 & 1.377327590 \\
\hline $\mathrm{H}$ & 5.761717880 & -1.964142820 & 1.077244230 \\
\hline $\mathrm{H}$ & 5.709572530 & -0.821199220 & -0.275938270 \\
\hline 0 & 2.340613080 & 3.332772840 & -0.865146130 \\
\hline $\mathrm{H}$ & 2.888548770 & 2.559974500 & -0.769276190 \\
\hline $\mathrm{H}$ & 2.549796010 & 3.875487550 & -0.117644170 \\
\hline $\mathrm{H}$ & -5.231174660 & -0.042573320 & 0.527418420 \\
\hline \multicolumn{4}{|c|}{ C1-C2-C3-C4 angle: 130} \\
\hline C & -2.877911710 & 0.723629390 & 0.417112710 \\
\hline C & -1.514975130 & 1.013867490 & 0.117801720 \\
\hline C & -0.628747630 & -0.041485560 & -0.367862940 \\
\hline C & -1.180222470 & -1.391367610 & -0.470529310 \\
\hline C & -2.570937170 & -1.619935620 & -0.179276680 \\
\hline C & -3.370796480 & -0.565377100 & 0.259974080 \\
\hline $\mathrm{H}$ & -3.521165790 & 1.506461450 & 0.777551590 \\
\hline
\end{tabular}




\begin{tabular}{|c|c|c|c|}
\hline $\mathrm{H}$ & -1.133987100 & 2.009504700 & 0.232992900 \\
\hline $\mathrm{H}$ & -0.573727050 & -2.186886190 & -0.854765730 \\
\hline $\mathrm{H}$ & -3.004957380 & -2.594857140 & -0.280836990 \\
\hline 0 & -4.653904310 & -0.862177400 & 0.527838580 \\
\hline C & 0.787206170 & 0.195112740 & -0.364524350 \\
\hline C & 1.717579930 & -0.655544930 & 0.157851690 \\
\hline $\mathrm{H}$ & 1.106592990 & 1.195711890 & -0.600164200 \\
\hline $\mathrm{H}$ & 1.465985280 & -1.639273330 & 0.503477250 \\
\hline C & 3.140628960 & -0.273680490 & 0.242025030 \\
\hline 0 & 3.608158260 & 0.771092130 & -0.108198000 \\
\hline 0 & 3.868815450 & -1.239363620 & 0.772066010 \\
\hline C & 5.258396950 & -1.001591240 & 0.917035330 \\
\hline $\mathrm{H}$ & 5.431768950 & -0.154474890 & 1.564600310 \\
\hline $\mathrm{H}$ & 5.664542410 & -1.898608220 & 1.356846580 \\
\hline $\mathrm{H}$ & 5.712709450 & -0.815686280 & -0.045124970 \\
\hline 0 & 2.425383100 & 3.358841640 & -0.970597220 \\
\hline $\mathrm{H}$ & 2.935492240 & 2.565048070 & -0.845957360 \\
\hline $\mathrm{H}$ & 2.608571430 & 3.889355600 & -0.207703350 \\
\hline $\mathrm{H}$ & -5.128462510 & -0.089100210 & 0.800888800 \\
\hline \multicolumn{4}{|c|}{ C1-C2-C3-C4 angle: 120} \\
\hline C & -2.874246300 & 0.671899030 & 0.549629190 \\
\hline C & -1.516637060 & 0.995281810 & 0.250925290 \\
\hline C & -0.645625670 & -0.005729340 & -0.358688030 \\
\hline C & -1.192729940 & -1.345087590 & -0.569098590 \\
\hline C & -2.572835620 & -1.607373800 & -0.270582260 \\
\hline C & -3.365247770 & -0.602682130 & 0.281090690 \\
\hline $\mathrm{H}$ & -3.513164900 & 1.412520270 & 0.996264070 \\
\hline $\mathrm{H}$ & -1.135788030 & 1.977219940 & 0.451649750 \\
\hline $\mathrm{H}$ & -0.585480770 & -2.099935180 & -1.027503800 \\
\hline $\mathrm{H}$ & -3.006698810 & -2.570611280 & -0.452784240 \\
\hline 0 & -4.640980310 & -0.926973310 & 0.547413620 \\
\hline C & 0.788143500 & 0.226233240 & -0.351090020 \\
\hline C & 1.692286370 & -0.594993040 & 0.237090590 \\
\hline $\mathrm{H}$ & 1.120308410 & 1.203213290 & -0.659819700 \\
\hline $\mathrm{H}$ & 1.418561570 & -1.549460580 & 0.644227290 \\
\hline C & 3.123360270 & -0.233153330 & 0.313928760 \\
\hline 0 & 3.615692770 & 0.773483740 & -0.105638590 \\
\hline 0 & 3.824552620 & -1.169761980 & 0.925203160 \\
\hline C & 5.216670230 & -0.948395130 & 1.073336850 \\
\hline $\mathrm{H}$ & 5.397932130 & -0.058757850 & 1.658696700 \\
\hline $\mathrm{H}$ & 5.598484220 & -1.817686420 & 1.584724770 \\
\hline $\mathrm{H}$ & 5.687713420 & -0.843484850 & 0.107008570 \\
\hline 0 & 2.517401910 & 3.273482120 & -1.275611260 \\
\hline $\mathrm{H}$ & 3.001609060 & 2.486584090 & -1.047496970 \\
\hline
\end{tabular}




\begin{tabular}{|c|c|c|c|}
\hline $\mathrm{H}$ & 2.705333510 & 3.887163660 & -0.579188720 \\
\hline $\mathrm{H}$ & -5.111102100 & -0.184159190 & 0.900491250 \\
\hline \multicolumn{4}{|c|}{ C1-C2-C3-C4 angle: 110} \\
\hline C & -2.883672580 & 0.604701190 & 0.699551200 \\
\hline C & -1.518311370 & 0.948907200 & 0.458223460 \\
\hline C & -0.658999870 & 0.022748700 & -0.271458690 \\
\hline C & -1.208684970 & -1.277432480 & -0.647602070 \\
\hline C & -2.590035170 & -1.563267500 & -0.394751500 \\
\hline C & -3.379411120 & -0.626211300 & 0.268501050 \\
\hline $\mathrm{H}$ & -3.524569100 & 1.288827020 & 1.226042320 \\
\hline $\mathrm{H}$ & -1.129724470 & 1.893723710 & 0.783122570 \\
\hline $\mathrm{H}$ & -0.597643660 & -1.975502240 & -1.184252190 \\
\hline $\mathrm{H}$ & -3.031223640 & -2.491691430 & -0.699100370 \\
\hline 0 & -4.659607010 & -0.966997330 & 0.480329230 \\
\hline C & 0.790162610 & 0.259450510 & -0.281665830 \\
\hline C & 1.680780520 & -0.520907220 & 0.363622920 \\
\hline $\mathrm{H}$ & 1.131139700 & 1.193063690 & -0.697867190 \\
\hline $\mathrm{H}$ & 1.391918470 & -1.430852580 & 0.854840350 \\
\hline C & 3.121230900 & -0.181735610 & 0.400291790 \\
\hline 0 & 3.629036760 & 0.775154180 & -0.106897700 \\
\hline 0 & 3.808510190 & -1.076696300 & 1.084423970 \\
\hline C & 5.206342210 & -0.873013260 & 1.202992150 \\
\hline $\mathrm{H}$ & 5.410869840 & 0.059525740 & 1.708400250 \\
\hline $\mathrm{H}$ & 5.574771680 & -1.703185520 & 1.784347540 \\
\hline $\mathrm{H}$ & 5.670503980 & -0.861360020 & 0.227764960 \\
\hline 0 & 2.586398250 & 3.109849930 & -1.619822640 \\
\hline $\mathrm{H}$ & 3.053484650 & 2.357325600 & -1.271805560 \\
\hline $\mathrm{H}$ & 2.774351520 & 3.815848290 & -1.017216800 \\
\hline $\mathrm{H}$ & -5.126933250 & -0.268833700 & 0.918184840 \\
\hline \multicolumn{4}{|c|}{ C1-C2-C3-C4 angle: 100} \\
\hline C & -2.914618150 & 0.494944330 & 0.901913440 \\
\hline C & -1.505908200 & 0.727408340 & 0.913617140 \\
\hline C & -0.646952690 & -0.088215760 & 0.067502540 \\
\hline C & -1.230606930 & -1.192681450 & -0.681994900 \\
\hline C & -2.647870810 & -1.381509630 & -0.658670270 \\
\hline C & -3.440436590 & -0.542354530 & 0.124006080 \\
\hline $\mathrm{H}$ & -3.570902540 & 1.113091590 & 1.487534930 \\
\hline $\mathrm{H}$ & -1.086479640 & 1.525966730 & 1.492480780 \\
\hline $\mathrm{H}$ & -0.605850630 & -1.802857290 & -1.303029080 \\
\hline $\mathrm{H}$ & -3.120106780 & -2.151395640 & -1.235872510 \\
\hline 0 & -4.758636710 & -0.775878040 & 0.098581340 \\
\hline C & 0.797775120 & 0.213874980 & -0.034456450 \\
\hline C & 1.738190250 & -0.461720130 & 0.647320100 \\
\hline $\mathrm{H}$ & 1.092751460 & 1.044040480 & -0.655882380 \\
\hline
\end{tabular}




\begin{tabular}{|c|c|c|c|}
\hline $\mathrm{H}$ & 1.493294490 & -1.283988270 & 1.293225350 \\
\hline C & 3.175501290 & -0.116924270 & 0.536886060 \\
\hline 0 & 3.634763390 & 0.762394060 & -0.130766500 \\
\hline 0 & 3.918853910 & -0.909807200 & 1.284525260 \\
\hline C & 5.319857920 & -0.692693600 & 1.271524580 \\
\hline $\mathrm{H}$ & 5.551572350 & 0.299952800 & 1.629031030 \\
\hline $\mathrm{H}$ & 5.737281120 & -1.436146950 & 1.931749550 \\
\hline $\mathrm{H}$ & 5.711581400 & -0.814380400 & 0.272342050 \\
\hline 0 & 2.472949350 & 2.710899820 & -2.051004960 \\
\hline $\mathrm{H}$ & 2.976773860 & 2.068883110 & -1.561576060 \\
\hline $\mathrm{H}$ & 2.621584680 & 3.532814750 & -1.604673630 \\
\hline $\mathrm{H}$ & -5.222602840 & -0.157930920 & 0.646887810 \\
\hline \multicolumn{4}{|c|}{ C1-C2-C3-C4 angle: 90} \\
\hline C & -2.893827290 & 0.368724420 & 1.044005370 \\
\hline C & -1.478035770 & 0.487623060 & 1.149192900 \\
\hline C & -0.630042960 & -0.225750560 & 0.204307890 \\
\hline C & -1.248951700 & -1.111046210 & -0.773997720 \\
\hline C & -2.677863910 & -1.184511670 & -0.844290270 \\
\hline C & -3.449739420 & -0.453221600 & 0.058946140 \\
\hline $\mathrm{H}$ & -3.534591350 & 0.918299670 & 1.709849520 \\
\hline $\mathrm{H}$ & -1.034422000 & 1.133263160 & 1.880814920 \\
\hline $\mathrm{H}$ & -0.637144450 & -1.639463650 & -1.477369580 \\
\hline $\mathrm{H}$ & -3.172286220 & -1.780709470 & -1.585205420 \\
\hline 0 & -4.777817750 & -0.572701090 & -0.062690050 \\
\hline C & 0.798906930 & 0.134097640 & 0.072259520 \\
\hline C & 1.771357310 & -0.433742500 & 0.805429580 \\
\hline $\mathrm{H}$ & 1.060048320 & 0.867149440 & -0.674230330 \\
\hline $\mathrm{H}$ & 1.562264090 & -1.182219890 & 1.546567380 \\
\hline C & 3.194054380 & -0.056221140 & 0.634384970 \\
\hline 0 & 3.614569950 & 0.756747670 & -0.135272750 \\
\hline 0 & 3.974101890 & -0.736523470 & 1.452522920 \\
\hline C & 5.366839710 & -0.479182240 & 1.389076120 \\
\hline $\mathrm{H}$ & 5.571430900 & 0.553956400 & 1.629120370 \\
\hline $\mathrm{H}$ & 5.818397620 & -1.130696390 & 2.120172820 \\
\hline $\mathrm{H}$ & 5.747175080 & -0.700027640 & 0.402575360 \\
\hline 0 & 2.375374760 & 2.340219450 & -2.325108040 \\
\hline $\mathrm{H}$ & 2.904949610 & 1.809911480 & -1.738720500 \\
\hline $\mathrm{H}$ & 2.462144220 & 3.226270710 & -2.002165190 \\
\hline $\mathrm{H}$ & -5.225940110 & -0.039693340 & 0.579868950 \\
\hline
\end{tabular}

\section{OMpCA}

C3-C4-C5-07 angle: 180

$\begin{array}{llll}C & -0.034920170 & 4.113934370 & 0.102814800 \\ C & -0.052962660 & 2.686905160 & 0.065660750\end{array}$




\begin{tabular}{|c|c|c|c|}
\hline C & 1.177597620 & 1.925844040 & 0.054972390 \\
\hline C & 2.428566610 & 2.643167540 & 0.082791430 \\
\hline C & 2.440232330 & 4.079780000 & 0.120149630 \\
\hline C & 1.214156060 & 4.793975920 & 0.129653380 \\
\hline $\mathrm{H}$ & -0.957263880 & 4.665288360 & 0.110327440 \\
\hline $\mathrm{H}$ & -0.991272910 & 2.166850270 & 0.045259460 \\
\hline $\mathrm{H}$ & 3.358621800 & 2.112919390 & 0.075887460 \\
\hline $\mathrm{H}$ & 3.362820730 & 4.624912280 & 0.141085370 \\
\hline 0 & 1.292385160 & 6.143421010 & 0.165235980 \\
\hline C & 1.095796910 & 0.484588820 & 0.016976030 \\
\hline C & 2.110975900 & -0.382530990 & 0.001928280 \\
\hline $\mathrm{H}$ & 0.104497510 & 0.065670140 & -0.001170710 \\
\hline $\mathrm{H}$ & 3.142009910 & -0.089194740 & 0.017104190 \\
\hline C & 1.839137880 & -1.833642390 & -0.037716520 \\
\hline 0 & 0.757514860 & -2.336337800 & -0.058695970 \\
\hline 0 & 2.965328610 & -2.534433680 & -0.047634100 \\
\hline C & 2.842595750 & -3.943585660 & -0.085098200 \\
\hline $\mathrm{H}$ & 2.320597570 & -4.255997220 & -0.978079740 \\
\hline $\mathrm{H}$ & 3.850895170 & -4.327575010 & -0.087661790 \\
\hline $\mathrm{H}$ & 2.307672870 & -4.301686090 & 0.782726920 \\
\hline $\mathrm{H}$ & 0.428544820 & 6.531004490 & 0.168939070 \\
\hline \multicolumn{4}{|c|}{ C3-C4-C5-07 angle: 170} \\
\hline C & 5.765999240 & 2.996311780 & 0.376665260 \\
\hline C & 5.258245460 & 1.663920790 & 0.306674210 \\
\hline C & 3.839984630 & 1.412996910 & 0.168207620 \\
\hline C & 2.946025910 & 2.542962970 & 0.102833380 \\
\hline C & 3.463165910 & 3.881877770 & 0.173927880 \\
\hline C & 4.860119690 & 4.090969480 & 0.309182230 \\
\hline $\mathrm{H}$ & 6.822082120 & 3.166707960 & 0.478791270 \\
\hline $\mathrm{H}$ & 5.935406880 & 0.833023590 & 0.355744310 \\
\hline $\mathrm{H}$ & 1.890546660 & 2.395074830 & 0.001120300 \\
\hline $\mathrm{H}$ & 2.809861700 & 4.730230930 & 0.126216560 \\
\hline 0 & 5.283704120 & 5.373631570 & 0.370308350 \\
\hline C & 3.386234990 & 0.043504810 & 0.100448890 \\
\hline C & 2.128492070 & -0.385910780 & -0.026034150 \\
\hline $\mathrm{H}$ & 4.150198690 & -0.712660520 & 0.155937960 \\
\hline $\mathrm{H}$ & 1.280861920 & 0.268709530 & -0.075524590 \\
\hline C & 1.844060060 & -1.835308520 & -0.044166830 \\
\hline 0 & 2.661712650 & -2.701571880 & -0.106499050 \\
\hline 0 & 0.541455550 & -2.071252540 & 0.038998190 \\
\hline C & 0.134371020 & -3.426541450 & 0.037125860 \\
\hline $\mathrm{H}$ & 0.556838630 & -3.951129670 & 0.881944090 \\
\hline $\mathrm{H}$ & -0.942207000 & -3.411234450 & 0.106645950 \\
\hline $\mathrm{H}$ & 0.443897560 & -3.915238920 & -0.875537320 \\
\hline
\end{tabular}




\begin{tabular}{|c|c|c|c|}
\hline $\mathrm{H}$ & 6.225525770 & 5.413581660 & 0.458887700 \\
\hline \multicolumn{4}{|c|}{ C3-C4-C5-07 angle: 160} \\
\hline C & 5.729097300 & 2.994824240 & 0.635854620 \\
\hline C & 5.227544070 & 1.662198440 & 0.532750860 \\
\hline C & 3.826706930 & 1.411288310 & 0.272006980 \\
\hline C & 2.943233790 & 2.540950030 & 0.119173690 \\
\hline C & 3.453803520 & 3.880148850 & 0.224293610 \\
\hline C & 4.833923370 & 4.089391780 & 0.479756320 \\
\hline $\mathrm{H}$ & 6.772416730 & 3.165622640 & 0.828426840 \\
\hline $\mathrm{H}$ & 5.896769320 & 0.831321430 & 0.647346530 \\
\hline $\mathrm{H}$ & 1.900665830 & 2.392836870 & -0.074148820 \\
\hline $\mathrm{H}$ & 2.808528690 & 4.728562310 & 0.112083130 \\
\hline 0 & 5.252316080 & 5.372229630 & 0.566870260 \\
\hline C & 3.378688450 & 0.040892760 & 0.175902190 \\
\hline C & 2.135954680 & -0.388550530 & -0.052992780 \\
\hline $\mathrm{H}$ & 4.135898020 & -0.714271500 & 0.298504140 \\
\hline $\mathrm{H}$ & 1.294362240 & 0.267133060 & -0.160782590 \\
\hline C & 1.847356850 & -1.837925010 & -0.052447230 \\
\hline 0 & 2.657619850 & -2.705537540 & -0.165761580 \\
\hline 0 & 0.554673790 & -2.070733520 & 0.132277650 \\
\hline C & 0.146211940 & -3.425323280 & 0.167292210 \\
\hline $\mathrm{H}$ & 0.627893180 & -3.945794190 & 0.982428250 \\
\hline $\mathrm{H}$ & -0.922453950 & -3.407357620 & 0.314540900 \\
\hline $\mathrm{H}$ & 0.388103270 & -3.919884050 & -0.762489090 \\
\hline $\mathrm{H}$ & 6.183000060 & 5.412289150 & 0.736249790 \\
\hline \multicolumn{4}{|c|}{ C3-C4-C5-07 angle: 150} \\
\hline C & 5.669403500 & 2.997018800 & 0.890069780 \\
\hline C & 5.178543900 & 1.663487700 & 0.753199100 \\
\hline C & 3.805913630 & 1.410347940 & 0.373020100 \\
\hline C & 2.939217780 & 2.538021850 & 0.134662090 \\
\hline C & 3.438711340 & 3.878208140 & 0.273868270 \\
\hline C & 4.791247290 & 4.089858600 & 0.647884830 \\
\hline $\mathrm{H}$ & 6.691837970 & 3.170030420 & 1.171946800 \\
\hline $\mathrm{H}$ & 5.835161600 & 0.833817950 & 0.931694610 \\
\hline $\mathrm{H}$ & 1.917698570 & 2.387937030 & -0.148645290 \\
\hline $\mathrm{H}$ & 2.806149930 & 4.725566030 & 0.098908840 \\
\hline 0 & 5.200541270 & 5.373459410 & 0.762103550 \\
\hline C & 3.367119830 & 0.037977480 & 0.249992590 \\
\hline C & 2.147981280 & -0.393334320 & -0.077276630 \\
\hline $\mathrm{H}$ & 4.113530040 & -0.714434980 & 0.438871160 \\
\hline $\mathrm{H}$ & 1.316387880 & 0.263051240 & -0.244604820 \\
\hline C & 1.852343420 & -1.842428440 & -0.059692580 \\
\hline 0 & 2.649022420 & -2.713179490 & -0.226990840 \\
\hline 0 & 0.577378010 & -2.068583500 & 0.227818140 \\
\hline
\end{tabular}




\begin{tabular}{|c|c|c|c|}
\hline C & 0.166142060 & -3.421315670 & 0.296111730 \\
\hline $\mathrm{H}$ & 0.701833690 & -3.941105460 & 1.077279630 \\
\hline $\mathrm{H}$ & -0.889329050 & -3.397678180 & 0.518430620 \\
\hline $\mathrm{H}$ & 0.339648970 & -3.920215400 & -0.646523840 \\
\hline $\mathrm{H}$ & 6.113051910 & 5.415113460 & 1.011196210 \\
\hline \multicolumn{4}{|c|}{ C3-C4-C5-07 angle: 140} \\
\hline C & 5.588622360 & 3.004074020 & 1.136833270 \\
\hline C & 5.111454410 & 1.668759170 & 0.971861340 \\
\hline C & 3.776654810 & 1.410819180 & 0.477892570 \\
\hline C & 2.933387940 & 2.534645350 & 0.153565680 \\
\hline C & 3.418698620 & 3.876706480 & 0.321166890 \\
\hline C & 4.734033460 & 4.093341680 & 0.808455910 \\
\hline $\mathrm{H}$ & 6.582945850 & 3.181298390 & 1.503832890 \\
\hline $\mathrm{H}$ & 5.750473470 & 0.841625900 & 1.214603880 \\
\hline $\mathrm{H}$ & 1.940707190 & 2.380637380 & -0.216874090 \\
\hline $\mathrm{H}$ & 2.803752630 & 4.721686080 & 0.083233880 \\
\hline 0 & 5.131771800 & 5.378412350 & 0.944422580 \\
\hline C & 3.350012070 & 0.035601600 & 0.331505080 \\
\hline C & 2.163048850 & -0.399505520 & -0.091291470 \\
\hline $\mathrm{H}$ & 4.081198040 & -0.712413640 & 0.586792870 \\
\hline $\mathrm{H}$ & 1.346109440 & 0.257007160 & -0.321069280 \\
\hline C & 1.858212070 & -1.848405250 & -0.063433470 \\
\hline 0 & 2.634400610 & -2.722515510 & -0.292952790 \\
\hline 0 & 0.609292220 & -2.067275790 & 0.325638760 \\
\hline C & 0.193677660 & -3.417724270 & 0.416515710 \\
\hline $\mathrm{H}$ & 0.785866630 & -3.947521070 & 1.148560920 \\
\hline $\mathrm{H}$ & -0.840382350 & -3.387706060 & 0.722671280 \\
\hline $\mathrm{H}$ & 0.287269830 & -3.910389200 & -0.540619090 \\
\hline $\mathrm{H}$ & 6.019655130 & 5.423442910 & 1.270151690 \\
\hline \multicolumn{4}{|c|}{ C3-C4-C5-07 angle: 130} \\
\hline C & 5.489928720 & 3.017539550 & 1.370757950 \\
\hline C & 5.028763500 & 1.679463340 & 1.184503070 \\
\hline C & 3.739237710 & 1.413239710 & 0.586418690 \\
\hline C & 2.923439940 & 2.530770700 & 0.181064610 \\
\hline C & 3.392082220 & 3.875742720 & 0.370412160 \\
\hline C & 4.663604850 & 4.100806850 & 0.959847840 \\
\hline $\mathrm{H}$ & 6.451078160 & 3.201630410 & 1.814681900 \\
\hline $\mathrm{H}$ & 5.647022750 & 0.856616350 & 1.488050950 \\
\hline $\mathrm{H}$ & 1.963200510 & 2.370356140 & -0.264583990 \\
\hline $\mathrm{H}$ & 2.797828410 & 4.716645100 & 0.072938340 \\
\hline 0 & 5.048033850 & 5.388169000 & 1.111050150 \\
\hline C & 3.327287010 & 0.033902330 & 0.422573100 \\
\hline C & 2.183061450 & -0.408176850 & -0.096207730 \\
\hline $\mathrm{H}$ & 4.036582610 & -0.707199410 & 0.750488590 \\
\hline
\end{tabular}




\begin{tabular}{|c|c|c|c|}
\hline $\mathrm{H}$ & 1.389025400 & 0.246849980 & -0.400391510 \\
\hline C & 1.866394600 & -1.857170980 & -0.063093800 \\
\hline 0 & 2.614785140 & -2.734954930 & -0.357409640 \\
\hline 0 & 0.649571650 & -2.068193160 & 0.420173230 \\
\hline C & 0.226147180 & -3.415635660 & 0.525148880 \\
\hline $\mathrm{H}$ & 0.866919860 & -3.961774610 & 1.202139480 \\
\hline $\mathrm{H}$ & -0.780668300 & -3.378571070 & 0.910836220 \\
\hline $\mathrm{H}$ & 0.239652790 & -3.895791950 & -0.442774190 \\
\hline $\mathrm{H}$ & 5.906877350 & 5.438815910 & 1.506378810 \\
\hline \multicolumn{4}{|c|}{ C3-C4-C5-07 angle: 120} \\
\hline C & 5.152616070 & 3.119888100 & 1.936380610 \\
\hline C & 4.755299740 & 1.764776000 & 1.727556150 \\
\hline C & 3.598549240 & 1.444677940 & 0.921248170 \\
\hline C & 2.850644430 & 2.524668410 & 0.327587060 \\
\hline C & 3.254582180 & 3.887058930 & 0.539715410 \\
\hline C & 4.394908350 & 4.165644750 & 1.338080900 \\
\hline $\mathrm{H}$ & 6.014582320 & 3.344937760 & 2.537332090 \\
\hline $\mathrm{H}$ & 5.322391850 & 0.969734690 & 2.172343210 \\
\hline $\mathrm{H}$ & 1.991404640 & 2.323454150 & -0.278782770 \\
\hline $\mathrm{H}$ & 2.709874350 & 4.701131670 & 0.104611830 \\
\hline 0 & 4.723532760 & 5.466891240 & 1.501183750 \\
\hline C & 3.244601580 & 0.049019380 & 0.747061640 \\
\hline C & 2.214817570 & -0.438784350 & 0.060225460 \\
\hline $\mathrm{H}$ & 3.902337910 & -0.659400890 & 1.222502760 \\
\hline $\mathrm{H}$ & 1.474302200 & 0.187740960 & -0.400337630 \\
\hline C & 1.916003310 & -1.894947210 & 0.090678900 \\
\hline 0 & 2.673816350 & -2.761879500 & -0.207719330 \\
\hline 0 & 0.701688250 & -2.121606010 & 0.572407110 \\
\hline C & 0.290856140 & -3.474221920 & 0.665545820 \\
\hline $\mathrm{H}$ & 0.937536530 & -4.020439450 & 1.336767790 \\
\hline $\mathrm{H}$ & -0.715747300 & -3.449843480 & 1.052689910 \\
\hline $\mathrm{H}$ & 0.307626800 & -3.945308800 & -0.306762300 \\
\hline $\mathrm{H}$ & 5.496052730 & 5.553664970 & 2.041686340 \\
\hline \multicolumn{4}{|c|}{ C3-C4-C5-07 angle: 110} \\
\hline C & 5.242598580 & 3.066948610 & 1.808615980 \\
\hline C & 4.809876660 & 1.722303940 & 1.602681140 \\
\hline C & 3.633468980 & 1.432374570 & 0.814061290 \\
\hline C & 2.900717790 & 2.531048960 & 0.236420920 \\
\hline C & 3.339741030 & 3.882916570 & 0.445532590 \\
\hline C & 4.499757840 & 4.131888880 & 1.225324680 \\
\hline $\mathrm{H}$ & 6.119497340 & 3.270149030 & 2.395596070 \\
\hline $\mathrm{H}$ & 5.365578560 & 0.912744550 & 2.035556340 \\
\hline $\mathrm{H}$ & 2.025537760 & 2.352313880 & -0.354038450 \\
\hline $\mathrm{H}$ & 2.807353410 & 4.711079530 & 0.021929450 \\
\hline
\end{tabular}




\begin{tabular}{|c|c|c|c|}
\hline 0 & 4.861950200 & 5.424454140 & 1.386087900 \\
\hline C & 3.244858310 & 0.044372070 & 0.640246760 \\
\hline C & 2.201919360 & -0.416438770 & -0.042493920 \\
\hline $\mathrm{H}$ & 3.889669460 & -0.677836010 & 1.113373670 \\
\hline $\mathrm{H}$ & 1.481431010 & 0.230005280 & -0.508282610 \\
\hline C & 1.869449850 & -1.869486410 & -0.043991140 \\
\hline 0 & 2.527471040 & -2.726550340 & -0.539136400 \\
\hline 0 & 0.755099000 & -2.104868040 & 0.633692410 \\
\hline C & 0.318990080 & -3.451370680 & 0.703620200 \\
\hline $\mathrm{H}$ & 1.056470080 & -4.062373540 & 1.203539650 \\
\hline $\mathrm{H}$ & -0.598943440 & -3.433822510 & 1.269920960 \\
\hline $\mathrm{H}$ & 0.141727970 & -3.846108150 & -0.286531040 \\
\hline $\mathrm{H}$ & 5.645521600 & 5.491115580 & 1.913372980 \\
\hline \multicolumn{4}{|c|}{ C3-C4-C5-07 angle: 100} \\
\hline C & 5.097337410 & 3.104747620 & 2.009221980 \\
\hline C & 4.668683670 & 1.757792640 & 1.809923580 \\
\hline C & 3.558160910 & 1.452686920 & 0.936425610 \\
\hline C & 2.886906470 & 2.537910440 & 0.266361860 \\
\hline C & 3.321155590 & 3.892232130 & 0.469076250 \\
\hline C & 4.415792150 & 4.156404170 & 1.333919450 \\
\hline $\mathrm{H}$ & 5.924962570 & 3.319780870 & 2.660066700 \\
\hline $\mathrm{H}$ & 5.178115710 & 0.958020430 & 2.312398540 \\
\hline $\mathrm{H}$ & 2.061313270 & 2.347765630 & -0.388468210 \\
\hline $\mathrm{H}$ & 2.834140210 & 4.710872190 & -0.022220970 \\
\hline 0 & 4.777130840 & 5.450463600 & 1.484056220 \\
\hline C & 3.170002540 & 0.061928910 & 0.774320480 \\
\hline C & 2.185089430 & -0.410516500 & 0.019656700 \\
\hline $\mathrm{H}$ & 3.763280460 & -0.647503970 & 1.327950330 \\
\hline $\mathrm{H}$ & 1.529342030 & 0.226295390 & -0.545186250 \\
\hline C & 1.857618250 & -1.867452590 & -0.023737460 \\
\hline 0 & 2.450991070 & -2.682962330 & -0.651500500 \\
\hline 0 & 0.817110850 & -2.152866160 & 0.744487760 \\
\hline C & 0.387509240 & -3.503479040 & 0.767132250 \\
\hline $\mathrm{H}$ & 1.171799030 & -4.144080700 & 1.143559960 \\
\hline $\mathrm{H}$ & -0.465843320 & -3.528988540 & 1.426421130 \\
\hline $\mathrm{H}$ & 0.106444430 & -3.829642160 & -0.223904430 \\
\hline $\mathrm{H}$ & 5.515235190 & 5.527448370 & 2.072041910 \\
\hline \multicolumn{4}{|c|}{ C3-C4-C5-07 angle: 90} \\
\hline C & 4.918284140 & 3.155328380 & 2.185475650 \\
\hline C & 4.511724830 & 1.802410470 & 2.021029680 \\
\hline C & 3.465156810 & 1.454071760 & 1.056193720 \\
\hline C & 2.872587820 & 2.541883590 & 0.287287860 \\
\hline C & 3.325145150 & 3.894485720 & 0.497117160 \\
\hline C & 4.328838430 & 4.157063330 & 1.434929860 \\
\hline
\end{tabular}




\begin{tabular}{lrrr}
$H$ & 5.687835360 & 3.397334220 & 2.897113060 \\
$\mathrm{H}$ & 4.969713050 & 1.026126900 & 2.602024510 \\
$\mathrm{H}$ & 2.108317800 & 2.350585770 & -0.436533330 \\
$\mathrm{H}$ & 2.904890460 & 4.709873610 & -0.057135430 \\
$\mathrm{O}$ & 4.691074770 & 5.445050790 & 1.567139490 \\
$\mathrm{C}$ & 3.083742590 & 0.109784090 & 0.918147390 \\
$\mathrm{C}$ & 2.118520060 & -0.388185940 & 0.059542930 \\
$\mathrm{H}$ & 3.597682490 & -0.601211940 & 1.543359250 \\
$\mathrm{H}$ & 1.556330020 & 0.239235990 & -0.604389700 \\
$\mathrm{C}$ & 1.817865750 & -1.848023410 & -0.019138750 \\
$\mathrm{O}$ & 2.349648620 & -2.604933120 & -0.764491220 \\
$\mathrm{O}$ & 0.870680460 & -2.206629290 & 0.833304620 \\
$\mathrm{C}$ & 0.486638430 & -3.571250670 & 0.829857520 \\
$\mathrm{H}$ & 1.327016250 & -4.202405410 & 1.080337080 \\
$\mathrm{H}$ & -0.285484530 & -3.660715410 & 1.577701980 \\
$\mathrm{H}$ & 0.104875600 & -3.855078080 & -0.140250680 \\
$\mathrm{H}$ & 5.374599910 & 5.536944800 & 2.216627240 \\
\hline
\end{tabular}

\section{OMpCA(W1)}

C3-C4-C5-07 angle: 180

$\begin{array}{lrrr}\text { C } & -0.543721420 & -4.686246570 & -0.130245780 \\ \text { C } & 0.668165350 & -3.927859530 & -0.101862430 \\ \text { C } & 0.651527000 & -2.478083680 & -0.063661230 \\ \text { C } & -0.635264430 & -1.821070030 & -0.055153990 \\ \text { C } & -1.850018790 & -2.593721240 & -0.083931710 \\ H & -1.794306810 & -4.010483310 & -0.120991770 \\ H & -0.509747420 & -5.758649740 & -0.158353810 \\ H & 1.612596180 & -4.437724530 & -0.108837500 \\ H & -0.697563520 & -0.752367150 & -0.027332630 \\ \text { O } & -2.806519430 & -2.109434750 & -0.077712480 \\ \mathrm{C} & -2.967002500 & -4.672731680 & -0.146562140 \\ \mathrm{C} & 1.895350930 & -1.774546640 & -0.036504640 \\ \mathrm{H} & 2.083623090 & -0.449374870 & -0.000188600 \\ \mathrm{H} & 2.784149380 & -2.382122760 & -0.046440890 \\ \mathrm{C} & 1.282258880 & 0.262661650 & 0.013109730 \\ \mathrm{O} & 3.451962290 & 0.100103790 & 0.023756300 \\ \mathrm{O} & 4.460150610 & -0.538174390 & 0.013834250 \\ \mathrm{C} & 3.438130270 & 1.427562900 & 0.058740830 \\ \mathrm{H} & 4.692570430 & 2.079679300 & 0.084612160 \\ \mathrm{H} & 5.266043670 & 1.837601040 & -0.798697380 \\ \mathrm{H} & 4.475763120 & 3.136472160 & 0.111038110 \\ \mathrm{H} & 5.253914810 & 1.791091830 & 0.961720470 \\ \mathrm{O} & -2.832976160 & -5.616009100 & -0.170562230 \\ \mathrm{H} & -2.703096850 & -7.561222810 & -0.221049210 \\ & -3.052465420 & -7.985824070 & -0.993705480\end{array}$




\begin{tabular}{|c|c|c|c|}
\hline $\mathrm{H}$ & -3.062906290 & -8.025885970 & 0.523240860 \\
\hline \multicolumn{4}{|c|}{ C3-C4-C5-07 angle: 170} \\
\hline C & -0.545009360 & -4.669326350 & -0.384986800 \\
\hline C & 0.667254510 & -3.913265650 & -0.324934840 \\
\hline C & 0.651298670 & -2.471755970 & -0.166606620 \\
\hline C & -0.634747440 & -1.820064830 & -0.072337990 \\
\hline C & -1.849872490 & -2.590189150 & -0.133904620 \\
\hline C & -1.795022200 & -3.999057800 & -0.288527110 \\
\hline $\mathrm{H}$ & -0.511749100 & -5.735774100 & -0.501608110 \\
\hline $\mathrm{H}$ & 1.611249680 & -4.418999020 & -0.396471830 \\
\hline $\mathrm{H}$ & -0.696290940 & -0.757270900 & 0.043917810 \\
\hline $\mathrm{H}$ & -2.805877250 & -2.109958740 & -0.063801860 \\
\hline 0 & -2.967950720 & -4.659556540 & -0.338692910 \\
\hline C & 1.896246780 & -1.770038480 & -0.110937550 \\
\hline C & 2.085253380 & -0.452367720 & 0.029652990 \\
\hline $\mathrm{H}$ & 2.784339080 & -2.373666870 & -0.189265340 \\
\hline $\mathrm{H}$ & 1.283877430 & 0.256305680 & 0.101121300 \\
\hline C & 3.453377830 & 0.099339890 & 0.030072370 \\
\hline 0 & 4.461732670 & -0.537531560 & 0.065816980 \\
\hline 0 & 3.438538180 & 1.425497060 & -0.035565810 \\
\hline C & 4.692407400 & 2.079401120 & -0.047949310 \\
\hline $\mathrm{H}$ & 5.271834350 & 1.775129990 & -0.907769700 \\
\hline $\mathrm{H}$ & 4.474584290 & 3.135060730 & -0.098990680 \\
\hline $\mathrm{H}$ & 5.248077180 & 1.855214760 & 0.851355840 \\
\hline $\mathrm{H}$ & -2.834376290 & -5.597508840 & -0.442287280 \\
\hline 0 & -2.708047940 & -7.532182660 & -0.650382560 \\
\hline $\mathrm{H}$ & -3.081764470 & -7.894149310 & -1.443266990 \\
\hline $\mathrm{H}$ & -3.048193160 & -8.052910640 & 0.065508540 \\
\hline \multicolumn{4}{|c|}{ C3-C4-C5-07 angle: 160} \\
\hline C & -0.562638330 & -4.628130100 & -0.633496350 \\
\hline C & 0.661998280 & -3.918588410 & -0.544850620 \\
\hline C & 0.685748310 & -2.479538850 & -0.265978400 \\
\hline C & -0.600894490 & -1.826576380 & -0.086798620 \\
\hline C & -1.820993540 & -2.598840950 & -0.188834160 \\
\hline C & -1.766459380 & -3.979657040 & -0.459404870 \\
\hline $\mathrm{H}$ & -0.551155230 & -5.682987210 & -0.838530110 \\
\hline $\mathrm{H}$ & 1.592338430 & -4.434936710 & -0.681234040 \\
\hline $\mathrm{H}$ & -0.661914040 & -0.779081790 & 0.123635170 \\
\hline $\mathrm{H}$ & -2.777370950 & -2.132486640 & -0.059264980 \\
\hline 0 & -2.945646370 & -4.611685110 & -0.534964420 \\
\hline C & 1.917984130 & -1.814206810 & -0.188304340 \\
\hline C & 2.106239050 & -0.464047210 & 0.058036790 \\
\hline $\mathrm{H}$ & 2.807444160 & -2.402829660 & -0.325895470 \\
\hline $\mathrm{H}$ & 1.294124820 & 0.225448380 & 0.169773610 \\
\hline
\end{tabular}




\begin{tabular}{|c|c|c|c|}
\hline C & 3.462935380 & 0.114417820 & 0.036845100 \\
\hline 0 & 4.477143650 & -0.503972880 & 0.132291120 \\
\hline 0 & 3.425424730 & 1.429559250 & -0.133895770 \\
\hline C & 4.668495870 & 2.104757100 & -0.177860930 \\
\hline $\mathrm{H}$ & 5.265368720 & 1.746921350 & -1.004314970 \\
\hline $\mathrm{H}$ & 4.432241880 & 3.148919940 & -0.311580850 \\
\hline $\mathrm{H}$ & 5.213596180 & 1.958916770 & 0.743624680 \\
\hline $\mathrm{H}$ & -2.832994100 & -5.541397960 & -0.717256540 \\
\hline 0 & -2.782676670 & -7.446144180 & -1.074556890 \\
\hline $\mathrm{H}$ & -3.178927590 & -7.747432330 & -1.881679320 \\
\hline $\mathrm{H}$ & -3.099686540 & -8.021501050 & -0.390653410 \\
\hline \multicolumn{4}{|c|}{ C3-C4-C5-07 angle: 150} \\
\hline C & -0.578693820 & -4.570884540 & -0.879685990 \\
\hline C & 0.650029730 & -3.870420330 & -0.762520840 \\
\hline C & 0.681818030 & -2.459081130 & -0.368057800 \\
\hline C & -0.599767640 & -1.823331970 & -0.105939130 \\
\hline C & -1.822476010 & -2.584370470 & -0.241496750 \\
\hline C & -1.777128180 & -3.937485010 & -0.624585830 \\
\hline $\mathrm{H}$ & -0.574601630 & -5.605482640 & -1.170129870 \\
\hline $\mathrm{H}$ & 1.575990370 & -4.373988700 & -0.961868400 \\
\hline $\mathrm{H}$ & -0.653662020 & -0.796796690 & 0.191791450 \\
\hline $\mathrm{H}$ & -2.774642940 & -2.130294250 & -0.051431450 \\
\hline 0 & -2.958370830 & -4.562202150 & -0.723764840 \\
\hline C & 1.917490370 & -1.800796820 & -0.265818070 \\
\hline C & 2.113037190 & -0.474896620 & 0.082753430 \\
\hline $\mathrm{H}$ & 2.802129130 & -2.378516860 & -0.467292110 \\
\hline $\mathrm{H}$ & 1.302472870 & 0.206025360 & 0.248129460 \\
\hline C & 3.468327410 & 0.111254240 & 0.042849710 \\
\hline 0 & 4.483304110 & -0.493415860 & 0.194411180 \\
\hline 0 & 3.426326520 & 1.408605990 & -0.229457900 \\
\hline C & 4.666307520 & 2.087405470 & -0.304939170 \\
\hline $\mathrm{H}$ & 5.277445820 & 1.673187670 & -1.093723440 \\
\hline $\mathrm{H}$ & 4.425964840 & 3.117054790 & -0.519326060 \\
\hline $\mathrm{H}$ & 5.198083710 & 2.013966760 & 0.632798590 \\
\hline $\mathrm{H}$ & -2.851111880 & -5.474148360 & -0.982735120 \\
\hline 0 & -2.815943590 & -7.345499040 & -1.489476790 \\
\hline $\mathrm{H}$ & -3.240124100 & -7.581635430 & -2.304055010 \\
\hline $\mathrm{H}$ & -3.115070850 & -7.970420950 & -0.841984840 \\
\hline \multicolumn{4}{|c|}{ C3-C4-C5-07 angle: 140} \\
\hline C & -0.603044400 & -4.494119650 & -1.120825850 \\
\hline C & 0.631145330 & -3.805810720 & -0.977991180 \\
\hline C & 0.675247730 & -2.430812440 & -0.472261620 \\
\hline C & -0.598825030 & -1.817763300 & -0.128891250 \\
\hline C & -1.825447860 & -2.564115130 & -0.295084810 \\
\hline
\end{tabular}




\begin{tabular}{|c|c|c|c|}
\hline C & -1.793469490 & -3.880942920 & -0.785959520 \\
\hline $\mathrm{H}$ & -0.609318040 & -5.502026290 & -1.493274830 \\
\hline $\mathrm{H}$ & 1.550678740 & -4.292458130 & -1.239370290 \\
\hline $\mathrm{H}$ & -0.642570040 & -0.818744860 & 0.252570980 \\
\hline $\mathrm{H}$ & -2.771409690 & -2.125939910 & -0.045739650 \\
\hline 0 & -2.977878420 & -4.495818130 & -0.906348470 \\
\hline C & 1.915664340 & -1.782698650 & -0.347686990 \\
\hline C & 2.122326080 & -0.489131200 & 0.102165270 \\
\hline $\mathrm{H}$ & 2.793081260 & -2.345412870 & -0.613802590 \\
\hline $\mathrm{H}$ & 1.314712450 & 0.179134570 & 0.325618420 \\
\hline C & 3.475600020 & 0.106990340 & 0.048168360 \\
\hline 0 & 4.490960130 & -0.477295310 & 0.259838820 \\
\hline 0 & 3.428949970 & 1.379050950 & -0.322610920 \\
\hline C & 4.665004710 & 2.062340560 & -0.425060340 \\
\hline $\mathrm{H}$ & 5.294182200 & 1.596582570 & -1.169621140 \\
\hline $\mathrm{H}$ & 4.420329920 & 3.070908420 & -0.719516040 \\
\hline $\mathrm{H}$ & 5.179187240 & 2.063301500 & 0.525267580 \\
\hline $\mathrm{H}$ & -2.878411900 & -5.384401390 & -1.238977430 \\
\hline 0 & -2.862447830 & -7.211974700 & -1.888902100 \\
\hline $\mathrm{H}$ & -3.314643080 & -7.384175390 & -2.704437970 \\
\hline $\mathrm{H}$ & -3.144669190 & -7.882585400 & -1.280710450 \\
\hline \multicolumn{4}{|c|}{ C3-C4-C5-07 angle: 130} \\
\hline C & -0.635108190 & -4.399381200 & -1.355475970 \\
\hline C & 0.605435320 & -3.725934260 & -1.191407320 \\
\hline C & 0.666755330 & -2.394714000 & -0.579820950 \\
\hline C & -0.597592460 & -1.809850830 & -0.156216980 \\
\hline C & -1.829193600 & -2.539152980 & -0.348680080 \\
\hline C & -1.814598760 & -3.812035040 & -0.941798010 \\
\hline $\mathrm{H}$ & -0.654685870 & -5.374771470 & -1.805860100 \\
\hline $\mathrm{H}$ & 1.516366570 & -4.191669820 & -1.513878180 \\
\hline $\mathrm{H}$ & -0.628379700 & -0.844230860 & 0.304458800 \\
\hline $\mathrm{H}$ & -2.766815420 & -2.120619110 & -0.040758780 \\
\hline 0 & -3.003581270 & -4.415175410 & -1.079643690 \\
\hline C & 1.912268440 & -1.759606910 & -0.436722040 \\
\hline C & 2.133322830 & -0.504858270 & 0.112660700 \\
\hline $\mathrm{H}$ & 2.780415390 & -2.302148910 & -0.767727900 \\
\hline $\mathrm{H}$ & 1.330073140 & 0.145363660 & 0.397914960 \\
\hline C & 3.484047260 & 0.103152890 & 0.051530660 \\
\hline 0 & 4.497672790 & -0.453719920 & 0.330536350 \\
\hline 0 & 3.435290340 & 1.342754000 & -0.414858890 \\
\hline C & 4.667136620 & 2.031720910 & -0.534481410 \\
\hline $\mathrm{H}$ & 5.321119060 & 1.519684240 & -1.225287830 \\
\hline $\mathrm{H}$ & 4.420383100 & 3.012622180 & -0.909411710 \\
\hline $\mathrm{H}$ & 5.154643570 & 2.109034210 & 0.426685290 \\
\hline
\end{tabular}




\begin{tabular}{|c|c|c|c|}
\hline $\mathrm{H}$ & -2.914514660 & -5.275166490 & -1.482716150 \\
\hline 0 & -2.924692680 & -7.049897370 & -2.269256110 \\
\hline $\mathrm{H}$ & -3.407044010 & -7.157657480 & -3.078548160 \\
\hline $\mathrm{H}$ & -3.193369870 & -7.760852520 & -1.702135550 \\
\hline \multicolumn{4}{|c|}{ C3-C4-C5-07 angle: 120} \\
\hline C & -0.671450760 & -4.287192210 & -1.578679800 \\
\hline C & 0.575350520 & -3.641408550 & -1.395427130 \\
\hline C & 0.664409800 & -2.355801430 & -0.682079920 \\
\hline C & -0.591169760 & -1.796912840 & -0.182877900 \\
\hline C & -1.833317780 & -2.506668580 & -0.403708980 \\
\hline C & -1.833280310 & -3.735234670 & -1.097901210 \\
\hline $\mathrm{H}$ & -0.707095620 & -5.225029310 & -2.103110870 \\
\hline $\mathrm{H}$ & 1.475025350 & -4.086122470 & -1.774364410 \\
\hline $\mathrm{H}$ & -0.605025920 & -0.870914010 & 0.353715550 \\
\hline $\mathrm{H}$ & -2.761888290 & -2.111362820 & -0.043055850 \\
\hline 0 & -3.034566420 & -4.318125260 & -1.252090080 \\
\hline C & 1.907782360 & -1.741065260 & -0.519823820 \\
\hline C & 2.150419810 & -0.519179890 & 0.133747540 \\
\hline $\mathrm{H}$ & 2.768078470 & -2.256441440 & -0.909730860 \\
\hline $\mathrm{H}$ & 1.350723910 & 0.110886870 & 0.469740930 \\
\hline C & 3.495513270 & 0.103354110 & 0.055995050 \\
\hline 0 & 4.507883960 & -0.420437280 & 0.395341980 \\
\hline 0 & 3.443324690 & 1.304716510 & -0.500102340 \\
\hline C & 4.669425290 & 2.001428370 & -0.638773040 \\
\hline $\mathrm{H}$ & 5.347231870 & 1.450452750 & -1.274403300 \\
\hline $\mathrm{H}$ & 4.419633380 & 2.949007020 & -1.089578240 \\
\hline $\mathrm{H}$ & 5.130822480 & 2.154716040 & 0.326144100 \\
\hline $\mathrm{H}$ & -2.960724190 & -5.145114090 & -1.720854370 \\
\hline 0 & -3.010100710 & -6.866757130 & -2.638526280 \\
\hline $\mathrm{H}$ & -3.529297720 & -6.905624880 & -3.431053320 \\
\hline $\mathrm{H}$ & -3.276430440 & -7.607294470 & -2.109466010 \\
\hline \multicolumn{4}{|c|}{ C3-C4-C5-07 angle: 110} \\
\hline C & -0.722673970 & -4.158714610 & -1.795320790 \\
\hline C & 0.530879310 & -3.521337890 & -1.602057380 \\
\hline C & 0.637318450 & -2.297704490 & -0.795976220 \\
\hline C & -0.598612330 & -1.783989190 & -0.213660380 \\
\hline C & -1.844564150 & -2.476320850 & -0.446210160 \\
\hline C & -1.869535320 & -3.643223950 & -1.230064820 \\
\hline $\mathrm{H}$ & -0.777633060 & -5.052932800 & -2.388848330 \\
\hline $\mathrm{H}$ & 1.418358120 & -3.931250180 & -2.043750820 \\
\hline $\mathrm{H}$ & -0.594089080 & -0.901314830 & 0.391622590 \\
\hline $\mathrm{H}$ & -2.760849140 & -2.112970210 & -0.025246680 \\
\hline 0 & -3.070509960 & -4.217909270 & -1.390841380 \\
\hline C & 1.887621650 & -1.690203280 & -0.626488950 \\
\hline
\end{tabular}




\begin{tabular}{|c|c|c|c|}
\hline C & 2.151850060 & -0.532765110 & 0.108748470 \\
\hline $\mathrm{H}$ & 2.731454540 & -2.174082530 & -1.087813770 \\
\hline $\mathrm{H}$ & 1.365718630 & 0.065502540 & 0.526489880 \\
\hline C & 3.498323970 & 0.095898520 & 0.051339270 \\
\hline 0 & 4.494944600 & -0.385067210 & 0.485725940 \\
\hline 0 & 3.467692530 & 1.250043260 & -0.599292210 \\
\hline C & 4.693794490 & 1.949107240 & -0.724274800 \\
\hline $\mathrm{H}$ & 5.410635200 & 1.359529530 & -1.277153090 \\
\hline $\mathrm{H}$ & 4.461770750 & 2.855999000 & -1.260293430 \\
\hline $\mathrm{H}$ & 5.099928670 & 2.182152570 & 0.249526980 \\
\hline $\mathrm{H}$ & -3.011505000 & -5.005567300 & -1.925459350 \\
\hline 0 & -3.090123580 & -6.643510110 & -2.970796880 \\
\hline $\mathrm{H}$ & -3.629705290 & -6.624307580 & -3.750347570 \\
\hline $\mathrm{H}$ & -3.334250990 & -7.427953180 & -2.497194090 \\
\hline \multicolumn{4}{|c|}{ C3-C4-C5-07 angle: 100} \\
\hline C & -0.787367230 & -4.012404340 & -2.007520190 \\
\hline C & 0.468871730 & -3.376018140 & -1.819968640 \\
\hline C & 0.599097130 & -2.220394000 & -0.924564110 \\
\hline C & -0.611935700 & -1.768428360 & -0.248460660 \\
\hline C & -1.860572830 & -2.454563970 & -0.478313680 \\
\hline C & -1.911570880 & -3.555049870 & -1.349816190 \\
\hline $\mathrm{H}$ & -0.863130120 & -4.857372700 & -2.666918510 \\
\hline $\mathrm{H}$ & 1.338862420 & -3.738472620 & -2.332286520 \\
\hline $\mathrm{H}$ & -0.586475110 & -0.935682760 & 0.423413270 \\
\hline $\mathrm{H}$ & -2.759899260 & -2.137395050 & 0.010987920 \\
\hline 0 & -3.111999380 & -4.130014850 & -1.502026740 \\
\hline C & 1.852346440 & -1.612615440 & -0.760285550 \\
\hline C & 2.141173770 & -0.520400560 & 0.051856830 \\
\hline $\mathrm{H}$ & 2.675320550 & -2.048584640 & -1.301222570 \\
\hline $\mathrm{H}$ & 1.376992870 & 0.021707720 & 0.574274530 \\
\hline C & 3.495221470 & 0.101429290 & 0.039911150 \\
\hline 0 & 4.449607440 & -0.332892240 & 0.599012320 \\
\hline 0 & 3.522105950 & 1.198821060 & -0.702572240 \\
\hline C & 4.756579770 & 1.889428830 & -0.782834650 \\
\hline $\mathrm{H}$ & 5.516599190 & 1.257566650 & -1.219109010 \\
\hline $\mathrm{H}$ & 4.574033620 & 2.745502350 & -1.413219710 \\
\hline $\mathrm{H}$ & 5.078792300 & 2.207837280 & 0.198210640 \\
\hline $\mathrm{H}$ & -3.071358760 & -4.871467490 & -2.100754690 \\
\hline 0 & -3.188278170 & -6.416279300 & -3.271970190 \\
\hline $\mathrm{H}$ & -3.758410000 & -6.335550320 & -4.025392650 \\
\hline $\mathrm{H}$ & -3.410220390 & -7.239528200 & -2.856918490 \\
\hline \multicolumn{4}{|c|}{ C3-C4-C5-07 angle: 90} \\
\hline C & -0.869512920 & -3.840159140 & -2.216284510 \\
\hline C & 0.379432060 & -3.182535460 & -2.052271190 \\
\hline
\end{tabular}




\begin{tabular}{|c|c|c|c|}
\hline$C$ & 0.536893680 & -2.106542240 & -1.067736960 \\
\hline$C$ & -0.637310140 & -1.753950370 & -0.277619380 \\
\hline C & -1.879629320 & -2.456579730 & -0.487556120 \\
\hline$C$ & -1.959323300 & -3.476944720 & -1.448818750 \\
\hline $\mathrm{H}$ & -0.967652690 & -4.625942370 & -2.942488510 \\
\hline $\mathrm{H}$ & 1.222693380 & -3.469537640 & -2.650064030 \\
\hline $\mathrm{H}$ & -0.589182240 & -0.981669760 & 0.461787390 \\
\hline $\mathrm{H}$ & -2.752939790 & -2.212247400 & 0.083803290 \\
\hline 0 & -3.151030740 & -4.074458940 & -1.575993080 \\
\hline$C$ & 1.782342300 & -1.473291870 & -0.931014230 \\
\hline C & 2.093286780 & -0.444295490 & -0.052575430 \\
\hline $\mathrm{H}$ & 2.573392790 & -1.825687770 & -1.571716700 \\
\hline $\mathrm{H}$ & 1.375626580 & -0.027541210 & 0.626829590 \\
\hline$C$ & 3.466429530 & 0.134596880 & 0.019666180 \\
\hline 0 & 4.324015630 & -0.252224620 & 0.745408330 \\
\hline 0 & 3.621712210 & 1.151507330 & -0.814536470 \\
\hline$C$ & 4.884877970 & 1.793608840 & -0.815756790 \\
\hline $\mathrm{H}$ & 5.664166500 & 1.097981000 & -1.091838790 \\
\hline $\mathrm{H}$ & 4.812034790 & 2.583799710 & -1.546440300 \\
\hline $\mathrm{H}$ & 5.103273520 & 2.203359470 & 0.159901330 \\
\hline $\mathrm{H}$ & -3.131074570 & -4.757407470 & -2.241766160 \\
\hline 0 & -3.291215900 & -6.188749100 & -3.543056000 \\
\hline $\mathrm{H}$ & -3.909512250 & -6.051324980 & -4.248797680 \\
\hline $\mathrm{H}$ & -3.471340800 & -7.052478800 & -3.195487120 \\
\hline \multicolumn{4}{|c|}{ OMpCA(W2) } \\
\hline \multicolumn{4}{|c|}{ C3-C4-C5-07 angle: 180} \\
\hline C & -1.176854600 & -4.787394300 & 0.075381560 \\
\hline C & 0.093592640 & -4.136339070 & 0.054951170 \\
\hline C & 0.189141800 & -2.692401010 & 0.025319110 \\
\hline C & -1.030238200 & -1.922300680 & 0.014917710 \\
\hline C & -2.305379630 & -2.585320940 & 0.034181250 \\
\hline C & -2.362990650 & -4.002693220 & 0.064677450 \\
\hline $\mathrm{H}$ & -1.231799440 & -5.860287620 & 0.100715160 \\
\hline $\mathrm{H}$ & 0.995431050 & -4.717675500 & 0.069578140 \\
\hline $\mathrm{H}$ & -0.997502960 & -0.852333020 & -0.010422240 \\
\hline $\mathrm{H}$ & -3.219217960 & -2.025181640 & 0.026753090 \\
\hline 0 & -3.593175330 & -4.564722660 & 0.082236220 \\
\hline C & 1.503567560 & -2.099349800 & 0.009239810 \\
\hline C & 1.801434680 & -0.795274550 & 0.008444500 \\
\hline $\mathrm{H}$ & 2.322838420 & -2.795230620 & -0.001960050 \\
\hline $\mathrm{H}$ & 1.062558930 & -0.018705660 & 0.020937190 \\
\hline C & 3.205509800 & -0.353339470 & -0.004387580 \\
\hline 0 & 4.170387500 & -1.065601780 & -0.015471570 \\
\hline 0 & 3.298986280 & 0.965539150 & -0.003188940 \\
\hline
\end{tabular}




\begin{tabular}{|c|c|c|c|}
\hline C & 4.599902430 & 1.524948600 & -0.008812570 \\
\hline $\mathrm{H}$ & 5.138520750 & 1.223794980 & -0.895596860 \\
\hline $\mathrm{H}$ & 4.459262830 & 2.594365650 & -0.001710050 \\
\hline $\mathrm{H}$ & 5.150611990 & 1.213233660 & 0.866708010 \\
\hline 0 & 4.466719590 & -4.019942140 & 0.020395790 \\
\hline $\mathrm{H}$ & 4.532690670 & -3.077244710 & 0.139934520 \\
\hline $\mathrm{H}$ & 4.835661380 & -4.183540890 & -0.836583030 \\
\hline $\mathrm{H}$ & -3.531027550 & -5.509139240 & 0.108584140 \\
\hline \multicolumn{4}{|c|}{ C3-C4-C5-07 angle: 170} \\
\hline C & -1.183539030 & -4.782231080 & -0.123167250 \\
\hline C & 0.087755150 & -4.133100720 & -0.095581890 \\
\hline C & 0.185098000 & -2.689613770 & -0.055038660 \\
\hline C & -1.033132850 & -1.917727020 & -0.045878460 \\
\hline C & -2.309099940 & -2.578666720 & -0.075820730 \\
\hline C & -2.368574660 & -3.995816700 & -0.113139880 \\
\hline $\mathrm{H}$ & -1.240037380 & -5.855056830 & -0.147890680 \\
\hline $\mathrm{H}$ & 0.988601270 & -4.716108550 & -0.091393820 \\
\hline $\mathrm{H}$ & -0.998888280 & -0.847763320 & -0.022340730 \\
\hline $\mathrm{H}$ & -3.222169600 & -2.017262420 & -0.069662900 \\
\hline 0 & -3.599472930 & -4.555990260 & -0.138756050 \\
\hline C & 1.500394280 & -2.098529180 & -0.023227950 \\
\hline C & 1.799311990 & -0.797041720 & 0.051600710 \\
\hline $\mathrm{H}$ & 2.319041420 & -2.794377180 & -0.058956930 \\
\hline $\mathrm{H}$ & 1.060787300 & -0.020442340 & 0.078825450 \\
\hline C & 3.203794660 & -0.355746620 & 0.041359070 \\
\hline 0 & 4.167504840 & -1.064837720 & 0.123074000 \\
\hline 0 & 3.299181780 & 0.956312260 & -0.091673890 \\
\hline C & 4.600485540 & 1.514257170 & -0.117004140 \\
\hline $\mathrm{H}$ & 5.161418680 & 1.129598020 & -0.956395750 \\
\hline $\mathrm{H}$ & 4.461069780 & 2.579172290 & -0.216983090 \\
\hline $\mathrm{H}$ & 5.128064810 & 1.289242580 & 0.798443460 \\
\hline 0 & 4.451098500 & -4.019328640 & 0.172729830 \\
\hline $\mathrm{H}$ & 4.499817760 & -3.079643710 & 0.320855620 \\
\hline $\mathrm{H}$ & 4.940762520 & -4.166389060 & -0.624666800 \\
\hline $\mathrm{H}$ & -3.538605170 & -5.500699320 & -0.155950100 \\
\hline \multicolumn{4}{|c|}{ C3-C4-C5-07 angle: 160} \\
\hline C & -1.189271690 & -4.761560960 & -0.314857160 \\
\hline C & 0.081975150 & -4.116342690 & -0.237750110 \\
\hline C & 0.179691270 & -2.676320220 & -0.131945970 \\
\hline C & -1.037584890 & -1.903240650 & -0.109467940 \\
\hline C & -2.313512280 & -2.559903210 & -0.189921440 \\
\hline C & -2.373528640 & -3.974077140 & -0.290257220 \\
\hline $\mathrm{H}$ & -1.246549240 & -5.832225710 & -0.386658740 \\
\hline $\mathrm{H}$ & 0.981898360 & -4.700752980 & -0.241132330 \\
\hline
\end{tabular}




\begin{tabular}{|c|c|c|c|}
\hline $\mathrm{H}$ & -1.002628980 & -0.835247410 & -0.040585090 \\
\hline $\mathrm{H}$ & -3.226083680 & -1.997852160 & -0.174885390 \\
\hline 0 & -3.604320340 & -4.530580950 & -0.360887970 \\
\hline C & 1.495480530 & -2.089035060 & -0.051086290 \\
\hline C & 1.793572390 & -0.793972230 & 0.095060160 \\
\hline $\mathrm{H}$ & 2.314015810 & -2.783885160 & -0.107084930 \\
\hline $\mathrm{H}$ & 1.054509590 & -0.018126460 & 0.133530970 \\
\hline C & 3.198727120 & -0.351844520 & 0.090453940 \\
\hline 0 & 4.157707230 & -1.048542340 & 0.270432180 \\
\hline 0 & 3.300521090 & 0.938369520 & -0.179954100 \\
\hline C & 4.602860190 & 1.493344080 & -0.220986290 \\
\hline $\mathrm{H}$ & 5.189403140 & 1.025353070 & -0.998203420 \\
\hline $\mathrm{H}$ & 4.468111040 & 2.541999080 & -0.434501920 \\
\hline $\mathrm{H}$ & 5.100914550 & 1.364724810 & 0.728992690 \\
\hline 0 & 4.426242220 & -4.004851340 & 0.304768830 \\
\hline $\mathrm{H}$ & 4.455891230 & -3.070754700 & 0.488723390 \\
\hline $\mathrm{H}$ & 5.015091070 & -4.128249200 & -0.426813560 \\
\hline $\mathrm{H}$ & -3.543929670 & -5.473733640 & -0.418214250 \\
\hline \multicolumn{4}{|c|}{ C3-C4-C5-07 angle: 150} \\
\hline C & -1.195819590 & -4.725293240 & -0.503990430 \\
\hline C & 0.074891980 & -4.086314180 & -0.380113130 \\
\hline C & 0.172373530 & -2.652720340 & -0.209622850 \\
\hline C & -1.043798810 & -1.878705800 & -0.171711220 \\
\hline C & -2.319185360 & -2.528490170 & -0.300125520 \\
\hline C & -2.379266070 & -3.936991750 & -0.462841550 \\
\hline $\mathrm{H}$ & -1.253729210 & -5.791800140 & -0.622143530 \\
\hline $\mathrm{H}$ & 0.973745630 & -4.672192530 & -0.392193870 \\
\hline $\mathrm{H}$ & -1.008390950 & -0.814528000 & -0.058226740 \\
\hline $\mathrm{H}$ & -3.231253800 & -1.966018080 & -0.274659700 \\
\hline 0 & -3.609488730 & -4.487589840 & -0.576065280 \\
\hline C & 1.488493780 & -2.071425830 & -0.081943140 \\
\hline C & 1.784339430 & -0.786979520 & 0.136814250 \\
\hline $\mathrm{H}$ & 2.307168070 & -2.764252580 & -0.160734840 \\
\hline $\mathrm{H}$ & 1.043848140 & -0.013120750 & 0.192130320 \\
\hline C & 3.190218650 & -0.342093990 & 0.137652960 \\
\hline 0 & 4.141443020 & -1.017065920 & 0.412828410 \\
\hline 0 & 3.302222190 & 0.912206420 & -0.264753010 \\
\hline C & 4.605986140 & 1.463153240 & -0.321249840 \\
\hline $\mathrm{H}$ & 5.215988030 & 0.919187570 & -1.027683690 \\
\hline $\mathrm{H}$ & 4.478990760 & 2.484036450 & -0.645333860 \\
\hline $\mathrm{H}$ & 5.073795190 & 1.433644630 & 0.652086570 \\
\hline 0 & 4.395208380 & -3.975090840 & 0.429302690 \\
\hline $\mathrm{H}$ & 4.402213950 & -3.047500950 & 0.645190390 \\
\hline $\mathrm{H}$ & 5.074174240 & -4.078958940 & -0.222890820 \\
\hline
\end{tabular}




\begin{tabular}{|c|c|c|c|}
\hline $\mathrm{H}$ & -3.549314270 & -5.427538420 & -0.672748920 \\
\hline \multicolumn{4}{|c|}{ C3-C4-C5-07 angle: 140} \\
\hline C & -1.203982060 & -4.674434460 & -0.688084070 \\
\hline C & 0.066169740 & -4.043682980 & -0.522975170 \\
\hline C & 0.163399870 & -2.619226430 & -0.289743330 \\
\hline C & -1.051469750 & -1.844477170 & -0.233766350 \\
\hline C & -2.326323990 & -2.485236600 & -0.404296690 \\
\hline C & -2.386515760 & -3.885713690 & -0.626814230 \\
\hline $\mathrm{H}$ & -1.262565880 & -5.734870960 & -0.851757250 \\
\hline $\mathrm{H}$ & 0.963878150 & -4.630869840 & -0.547724660 \\
\hline $\mathrm{H}$ & -1.015459700 & -0.785623280 & -0.078260760 \\
\hline $\mathrm{H}$ & -3.237834540 & -1.922599940 & -0.365915520 \\
\hline 0 & -3.616152940 & -4.428595650 & -0.777038530 \\
\hline C & 1.479992170 & -2.045900060 & -0.119928090 \\
\hline C & 1.772787910 & -0.776664930 & 0.172511840 \\
\hline $\mathrm{H}$ & 2.299052650 & -2.735099450 & -0.226077150 \\
\hline $\mathrm{H}$ & 1.029945100 & -0.006814230 & 0.253596660 \\
\hline C & 3.179321090 & -0.326503030 & 0.180249490 \\
\hline 0 & 4.119702430 & -0.969694590 & 0.549501750 \\
\hline 0 & 3.305181330 & 0.878930090 & -0.347185130 \\
\hline C & 4.610254360 & 1.426505000 & -0.414479480 \\
\hline $\mathrm{H}$ & 5.243696460 & 0.816610590 & -1.041983300 \\
\hline $\mathrm{H}$ & 4.493865310 & 2.409221410 & -0.843422020 \\
\hline $\mathrm{H}$ & 5.044847970 & 1.496330570 & 0.572110530 \\
\hline 0 & 4.356296060 & -3.930981010 & 0.544496390 \\
\hline $\mathrm{H}$ & 4.339678550 & -3.010337180 & 0.787391490 \\
\hline $\mathrm{H}$ & 5.117126310 & -4.023040280 & -0.012086580 \\
\hline $\mathrm{H}$ & -3.556168500 & -5.363737990 & -0.912625940 \\
\hline \multicolumn{4}{|c|}{ C3-C4-C5-07 angle: 130} \\
\hline C & -1.215365170 & -4.610395140 & -0.866781960 \\
\hline C & 0.054405080 & -3.989414660 & -0.665571220 \\
\hline C & 0.151622710 & -2.576650680 & -0.371049450 \\
\hline C & -1.061981120 & -1.802127680 & -0.291938200 \\
\hline C & -2.336555420 & -2.432258520 & -0.499153040 \\
\hline C & -2.396992060 & -3.822217060 & -0.780884830 \\
\hline $\mathrm{H}$ & -1.274846030 & -5.662961700 & -1.074943210 \\
\hline $\mathrm{H}$ & 0.951002990 & -4.577351950 & -0.706537090 \\
\hline $\mathrm{H}$ & -1.025159420 & -0.750055200 & -0.095671160 \\
\hline $\mathrm{H}$ & -3.247534740 & -1.870084520 & -0.444693070 \\
\hline 0 & -3.626225020 & -4.356012170 & -0.963159590 \\
\hline C & 1.468967700 & -2.012704360 & -0.164957630 \\
\hline C & 1.758495690 & -0.762493590 & 0.198076850 \\
\hline $\mathrm{H}$ & 2.288497020 & -2.696697530 & -0.300791670 \\
\hline $\mathrm{H}$ & 1.012652490 & 0.001014360 & 0.311188130 \\
\hline
\end{tabular}




\begin{tabular}{|c|c|c|c|}
\hline C & 3.165682110 & -0.304903950 & 0.218013300 \\
\hline 0 & 4.089536010 & -0.903728060 & 0.687143150 \\
\hline 0 & 3.312231680 & 0.837537260 & -0.429713720 \\
\hline C & 4.618426730 & 1.383258960 & -0.499228310 \\
\hline $\mathrm{H}$ & 5.278694880 & 0.714550470 & -1.031943380 \\
\hline $\mathrm{H}$ & 4.518518210 & 2.314644860 & -1.033745170 \\
\hline $\mathrm{H}$ & 5.010621010 & 1.558990650 & 0.491946470 \\
\hline 0 & 4.315601570 & -3.869313670 & 0.644426110 \\
\hline $\mathrm{H}$ & 4.273367650 & -2.957844510 & 0.916361560 \\
\hline $\mathrm{H}$ & 5.145219980 & -3.952311360 & 0.195056220 \\
\hline $\mathrm{H}$ & -3.566509100 & -5.284806090 & -1.137094580 \\
\hline \multicolumn{4}{|c|}{ C3-C4-C5-07 angle: 120} \\
\hline C & -1.232164040 & -4.534669070 & -1.038363140 \\
\hline C & 0.038029760 & -3.924069810 & -0.809796970 \\
\hline C & 0.136168320 & -2.525385410 & -0.456070050 \\
\hline C & -1.076082110 & -1.752618980 & -0.347029370 \\
\hline C & -2.351251060 & -2.371507570 & -0.581714770 \\
\hline C & -2.412791210 & -3.748715090 & -0.920893300 \\
\hline $\mathrm{H}$ & -1.293178670 & -5.577626120 & -1.289897080 \\
\hline $\mathrm{H}$ & 0.933626470 & -4.511470650 & -0.873310430 \\
\hline $\mathrm{H}$ & -1.037838820 & -0.708786520 & -0.111001330 \\
\hline $\mathrm{H}$ & -3.261682360 & -1.810957270 & -0.505856630 \\
\hline 0 & -3.642389560 & -4.273008860 & -1.126462060 \\
\hline C & 1.455122220 & -1.971199170 & -0.223121800 \\
\hline C & 1.742191070 & -0.743621030 & 0.207148530 \\
\hline $\mathrm{H}$ & 2.274934240 & -2.647557180 & -0.393997720 \\
\hline $\mathrm{H}$ & 0.993106940 & 0.009720080 & 0.363410960 \\
\hline C & 3.150025740 & -0.277271290 & 0.247723750 \\
\hline 0 & 4.047351490 & -0.816526260 & 0.825443650 \\
\hline 0 & 3.328800130 & 0.787905520 & -0.512775480 \\
\hline C & 4.635949720 & 1.333745430 & -0.571582330 \\
\hline $\mathrm{H}$ & 5.325455270 & 0.616127640 & -0.991662310 \\
\hline $\mathrm{H}$ & 4.562750440 & 2.200139570 & -1.209602150 \\
\hline $\mathrm{H}$ & 4.973431810 & 1.620015800 & 0.413959210 \\
\hline 0 & 4.272658230 & -3.787311340 & 0.728272850 \\
\hline $\mathrm{H}$ & 4.202109000 & -2.888044610 & 1.032136000 \\
\hline $\mathrm{H}$ & 5.156863530 & -3.865811650 & 0.398072650 \\
\hline $\mathrm{H}$ & -3.583355920 & -5.193762180 & -1.339029680 \\
\hline \multicolumn{4}{|c|}{ C3-C4-C5-07 angle: 110} \\
\hline C & -1.255792820 & -4.450047500 & -1.202387530 \\
\hline C & 0.015656550 & -3.848376570 & -0.957433440 \\
\hline C & 0.115417060 & -2.465524400 & -0.547770120 \\
\hline C & -1.095813030 & -1.697811910 & -0.400559980 \\
\hline C & -2.372451400 & -2.306875870 & -0.651646990 \\
\hline
\end{tabular}




\begin{tabular}{|c|c|c|c|}
\hline C & -2.435693310 & -3.669620470 & -1.045336700 \\
\hline $\mathrm{H}$ & -1.318721560 & -5.482016630 & -1.495414790 \\
\hline $\mathrm{H}$ & 0.910647760 & -4.432745000 & -1.050117260 \\
\hline $\mathrm{H}$ & -1.055728270 & -0.663206710 & -0.127069030 \\
\hline $\mathrm{H}$ & -3.282599610 & -1.750305080 & -0.548243850 \\
\hline 0 & -3.666386580 & -4.185984670 & -1.263753920 \\
\hline C & 1.436764880 & -1.919109670 & -0.299460290 \\
\hline C & 1.722380080 & -0.716634030 & 0.192848040 \\
\hline $\mathrm{H}$ & 2.256816870 & -2.583692940 & -0.512089490 \\
\hline $\mathrm{H}$ & 0.970266730 & 0.020162430 & 0.406274720 \\
\hline C & 3.130641010 & -0.242309120 & 0.268550640 \\
\hline 0 & 3.982317160 & -0.702157980 & 0.968955130 \\
\hline 0 & 3.362414120 & 0.729496580 & -0.594558010 \\
\hline C & 4.670746720 & 1.275993920 & -0.623477340 \\
\hline $\mathrm{H}$ & 5.388662540 & 0.520005440 & -0.906188390 \\
\hline $\mathrm{H}$ & 4.643348060 & 2.061145960 & -1.362217940 \\
\hline $\mathrm{H}$ & 4.936393630 & 1.678268230 & 0.343329710 \\
\hline 0 & 4.229887470 & -3.677796500 & 0.790866680 \\
\hline $\mathrm{H}$ & 4.124371430 & -2.797107150 & 1.135274620 \\
\hline $\mathrm{H}$ & 5.152287830 & -3.754324510 & 0.590124690 \\
\hline $\mathrm{H}$ & -3.608245500 & -5.097501100 & -1.513284320 \\
\hline \multicolumn{4}{|c|}{ C3-C4-C5-07 angle: 100} \\
\hline C & -1.262786390 & -4.434279190 & -1.266163520 \\
\hline C & 0.008467460 & -3.823808650 & -1.043024430 \\
\hline C & 0.104067750 & -2.458099520 & -0.579550350 \\
\hline C & -1.110773710 & -1.714477430 & -0.358123920 \\
\hline C & -2.387521030 & -2.331455140 & -0.587785450 \\
\hline C & -2.446420050 & -3.678195160 & -1.034163880 \\
\hline $\mathrm{H}$ & -1.322905460 & -5.454294570 & -1.598965670 \\
\hline $\mathrm{H}$ & 0.907120970 & -4.390470170 & -1.192917460 \\
\hline $\mathrm{H}$ & -1.074309380 & -0.689808700 & -0.048734240 \\
\hline $\mathrm{H}$ & -3.300788720 & -1.793061400 & -0.429871270 \\
\hline 0 & -3.677172660 & -4.203842450 & -1.228700930 \\
\hline C & 1.426908440 & -1.901344690 & -0.354699720 \\
\hline C & 1.710194670 & -0.725173060 & 0.194712980 \\
\hline $\mathrm{H}$ & 2.248422610 & -2.536780490 & -0.639503980 \\
\hline $\mathrm{H}$ & 0.954420550 & -0.026867230 & 0.504502860 \\
\hline C & 3.115103590 & -0.234510640 & 0.299153780 \\
\hline 0 & 3.909574840 & -0.589277040 & 1.116759730 \\
\hline 0 & 3.404111070 & 0.633871310 & -0.651078110 \\
\hline C & 4.709574870 & 1.188629020 & -0.650434160 \\
\hline $\mathrm{H}$ & 5.448547680 & 0.413175740 & -0.790171630 \\
\hline $\mathrm{H}$ & 4.733006670 & 1.881282680 & -1.476610720 \\
\hline $\mathrm{H}$ & 4.902742390 & 1.704361180 & 0.278945400 \\
\hline
\end{tabular}




\begin{tabular}{|c|c|c|c|}
\hline 0 & 4.261577670 & -3.554165750 & 0.771199840 \\
\hline $\mathrm{H}$ & 4.109478680 & -2.713312310 & 1.188625390 \\
\hline $\mathrm{H}$ & 5.202670640 & -3.622949740 & 0.689982540 \\
\hline $\mathrm{H}$ & -3.615725320 & -5.103221830 & -1.518168230 \\
\hline \multicolumn{4}{|c|}{ C3-C4-C5-07 angle: 90} \\
\hline C & -1.497153730 & -3.927511240 & -2.107755250 \\
\hline C & -0.196942640 & -3.350765500 & -1.988828550 \\
\hline C & 0.085581650 & -2.357916250 & -0.977026650 \\
\hline C & -0.971834900 & -1.962362620 & -0.081447680 \\
\hline C & -2.278693280 & -2.546536270 & -0.203291620 \\
\hline C & -2.523370420 & -3.516499110 & -1.210866760 \\
\hline $\mathrm{H}$ & -1.696099040 & -4.663287090 & -2.865227360 \\
\hline $\mathrm{H}$ & 0.583200500 & -3.656238770 & -2.659433880 \\
\hline $\mathrm{H}$ & -0.790394240 & -1.248987080 & 0.696150100 \\
\hline $\mathrm{H}$ & -3.074195670 & -2.268669630 & 0.458940300 \\
\hline 0 & -3.773487590 & -4.026617070 & -1.277234910 \\
\hline C & 1.425274980 & -1.799960070 & -0.913100730 \\
\hline C & 1.858334040 & -0.855032730 & -0.087323200 \\
\hline $\mathrm{H}$ & 2.125920050 & -2.211074090 & -1.621750470 \\
\hline $\mathrm{H}$ & 1.230424260 & -0.390359390 & 0.650275920 \\
\hline C & 3.274993570 & -0.382560680 & -0.092915550 \\
\hline 0 & 4.141365450 & -0.842167930 & 0.586890430 \\
\hline 0 & 3.469230550 & 0.627191840 & -0.916519160 \\
\hline C & 4.776359810 & 1.178311560 & -0.973738390 \\
\hline $\mathrm{H}$ & 5.486744860 & 0.432420350 & -1.298834370 \\
\hline $\mathrm{H}$ & 4.722059260 & 1.982928930 & -1.689434850 \\
\hline $\mathrm{H}$ & 5.069838040 & 1.554899940 & -0.004815250 \\
\hline 0 & 3.854919560 & -3.016647560 & 2.673444940 \\
\hline $\mathrm{H}$ & 3.882968810 & -2.379501710 & 1.967974300 \\
\hline $\mathrm{H}$ & 4.691848220 & -2.935410490 & 3.108434730 \\
\hline $\mathrm{H}$ & -3.834986070 & -4.678952850 & -1.960695690 \\
\hline
\end{tabular}

LIIC path connecting the ${ }^{1} \pi \pi^{*}$ minima and the ${ }^{1} n \pi^{*}$ minima of OMpCA (10 points)

\section{Coord.1}

$\begin{array}{lrrr}\mathrm{C} & 0.064750500 & 4.144636000 & 0.104216700 \\ \mathrm{C} & 0.024941700 & 2.772893400 & 0.068250100 \\ \mathrm{C} & 1.197113900 & 1.989559600 & 0.056660200 \\ \mathrm{C} & 2.422421000 & 2.696014000 & 0.084211600 \\ \mathrm{C} & 2.467225400 & 4.063117300 & 0.120097200 \\ \mathrm{C} & 1.287266600 & 4.808706500 & 0.130645400 \\ \mathrm{H} & -0.854140700 & 4.707756400 & 0.111974000 \\ \mathrm{H} & -0.932193800 & 2.281315200 & 0.048298800 \\ \mathrm{H} & 3.351976200 & 2.157727400 & 0.077177900 \\ \mathrm{H} & 3.406847200 & 4.584226900 & 0.140688400\end{array}$




\begin{tabular}{|c|c|c|c|}
\hline 0 & 1.393158100 & 6.160427700 & 0.166586500 \\
\hline$C$ & 1.091776300 & 0.551841700 & 0.018502400 \\
\hline$C$ & 2.188670100 & -0.369800600 & 0.002627000 \\
\hline $\mathrm{H}$ & 0.095365900 & 0.148807600 & 0.000679400 \\
\hline$H$ & 3.202015700 & -0.018933300 & 0.019093300 \\
\hline$C$ & 2.068390300 & -1.716608100 & -0.033098900 \\
\hline 0 & 0.847687200 & -2.359524100 & -0.058230700 \\
\hline 0 & 3.116954300 & -2.554321400 & -0.047495500 \\
\hline$C$ & 2.909498100 & -3.949101900 & -0.084686800 \\
\hline $\mathrm{H}$ & 2.379705400 & -4.245660400 & -0.981741100 \\
\hline $\mathrm{H}$ & 3.893650300 & -4.389829900 & -0.089538600 \\
\hline $\mathrm{H}$ & 2.368041000 & -4.291136300 & 0.788907900 \\
\hline $\mathrm{H}$ & 0.535121500 & 6.560157700 & 0.170561200 \\
\hline \multicolumn{4}{|c|}{ Coord.2 } \\
\hline$C$ & 0.038942300 & 4.145908700 & 0.104150900 \\
\hline$C$ & 0.017430000 & 2.712381800 & 0.066804400 \\
\hline$C$ & 1.247376400 & 1.934163600 & 0.055683200 \\
\hline$C$ & 2.497127800 & 2.666444800 & 0.083897100 \\
\hline$C$ & 2.504314600 & 4.111268600 & 0.121431500 \\
\hline$C$ & 1.282710300 & 4.827886400 & 0.131015900 \\
\hline $\mathrm{H}$ & -0.885693300 & 4.693462700 & 0.111535000 \\
\hline $\mathrm{H}$ & -0.924375600 & 2.198906000 & 0.046535500 \\
\hline $\mathrm{H}$ & 3.431588600 & 2.144289200 & 0.077250600 \\
\hline $\mathrm{H}$ & 3.429055500 & 4.652866600 & 0.142299700 \\
\hline 0 & 1.363282200 & 6.178925500 & 0.166655500 \\
\hline$C$ & 1.166624400 & 0.527484100 & 0.018590400 \\
\hline$C$ & 2.182861800 & -0.352017600 & 0.003245100 \\
\hline $\mathrm{H}$ & 0.176138400 & 0.105221200 & 0.000346700 \\
\hline $\mathrm{H}$ & 3.215386200 & -0.064361400 & 0.018293000 \\
\hline$C$ & 1.903749400 & -1.799307500 & -0.036353700 \\
\hline 0 & 0.820389700 & -2.299102300 & -0.057259900 \\
\hline 0 & 3.026934100 & -2.506450100 & -0.046474200 \\
\hline$C$ & 2.896638100 & -3.914555600 & -0.083967400 \\
\hline $\mathrm{H}$ & 2.372857900 & -4.224527900 & -0.976798800 \\
\hline $\mathrm{H}$ & 3.902865000 & -4.304148700 & -0.086692700 \\
\hline $\mathrm{H}$ & 2.359941000 & -4.270211600 & 0.783812100 \\
\hline $\mathrm{H}$ & 0.500096900 & 6.567744800 & 0.170386400 \\
\hline \multicolumn{4}{|c|}{ Coord.3 } \\
\hline$C$ & 0.041757800 & 4.146432300 & 0.104173300 \\
\hline$C$ & 0.017959000 & 2.719760800 & 0.066979700 \\
\hline$C$ & 1.241373200 & 1.940720900 & 0.055800800 \\
\hline$C$ & 2.488489800 & 2.669994500 & 0.083937900 \\
\hline$C$ & 2.500190500 & 4.106232100 & 0.121291000 \\
\hline C & 1.283246900 & 4.826278400 & 0.130986400 \\
\hline
\end{tabular}


H

$\mathrm{H}$

$\mathrm{H}$

$\mathrm{H}$

0

C

C

$\mathrm{H}$

$\mathrm{H}$

C

0

0

C

$\mathrm{H}$

$\mathrm{H}$

$\mathrm{H}$

$\mathrm{H}$

\section{Coord.4}

C

C

C

C

C

C

$\mathrm{H}$

$\mathrm{H}$

H

$\mathrm{H}$

0

C

C

$\mathrm{H}$

$\mathrm{H}$

C

0

0

C

$\mathrm{H}$

$\mathrm{H}$

$\mathrm{H}$

$\mathrm{H}$

\section{Coord.5}

C

C

-0.882187600
-0.925635000
3.422369600
3.426652800
1.366775700
1.157782100
2.182810600
0.166572600
3.213430200
1.922281200
0.823026500
3.037870300
2.898798500
2.374302000
3.902658400
2.361518600
0.504197900

4.695827100

0.111601200

$-1.790822700$

$-2.305602000$

$-2.512931500$

$-3.919750700$

$-4.228162900$

$-4.315195700$

$-4.273827900$

0.044586500

$-0.054074400$

$-1.782156500$

$-2.312148900$

$-3.924604300$

$-4.231473400$

$-4.325859000$

$-4.277118600$

2.362926900

0.508247600

0.047428200

0.019248500
2.208807100

2.145957100

4.645495000

6.177407600

0.530581200

$-0.353823500$

0.110561300

$-0.059203300$

6.567536100

4.146787600

2.726974600

1.947177000

2.673477700

4.101115500

4.824537600

4.697995800

2.218521400

2.147580700

4.638057400

6.175752800

0.533580600

$-0.355674200$

0.115777600

$-2.519121900$

6.567166400

0.046748400

0.077246700

0.142126800

0.166658900

0.018590900

0.003181900

0.000396600

0.018386900

$-0.035998000$

$-0.057361300$

$-0.046607500$

$-0.084082500$

$-0.977391400$

$-0.087048900$

0.784349800

0.170419000

0.104191900

0.067151200

0.055916100

0.083977200

0.121148600

0.130953900

0.111662900

0.046956900

0.077241800

0.141952400

0.166659500

0.018588800

0.003117200

0.000443300

0.018479400

$-0.035640700$

$-0.057464100$

$-0.046736000$

$-0.084188700$

$-0.977972900$

$-0.087395200$

0.784894800

0.170448100

4. 146975400

0.104206600

2.734023500

0.067319100 


\begin{tabular}{|c|c|c|c|}
\hline C & 1.229681200 & 1.953532300 & 0.056029200 \\
\hline C & 2.471466500 & 2.676894500 & 0.084015000 \\
\hline C & 2.491942200 & 4.095919500 & 0.121004200 \\
\hline C & 1.284297500 & 4.822664800 & 0.130918500 \\
\hline $\mathrm{H}$ & -0.875174100 & 4.699969400 & 0.111720300 \\
\hline $\mathrm{H}$ & -0.927857800 & 2.228049200 & 0.047161200 \\
\hline $\mathrm{H}$ & 3.404210200 & 2.149160200 & 0.077235900 \\
\hline $\mathrm{H}$ & 3.421795800 & 4.630554800 & 0.141776400 \\
\hline 0 & 1.373633800 & 6.173962100 & 0.166657300 \\
\hline C & 1.140489000 & 0.536482200 & 0.018584200 \\
\hline C & 2.183225000 & -0.357568700 & 0.003051200 \\
\hline $\mathrm{H}$ & 0.147882300 & 0.120869800 & 0.000486700 \\
\hline $\mathrm{H}$ & 3.209868600 & -0.048974000 & 0.018570700 \\
\hline C & 1.959181100 & -1.773311200 & -0.035281800 \\
\hline 0 & 0.828593400 & -2.318745800 & -0.057568300 \\
\hline 0 & 3.059044200 & -2.525021400 & -0.046859700 \\
\hline C & 2.902570000 & -3.929117300 & -0.084286100 \\
\hline $\mathrm{H}$ & 2.376677400 & -4.234460700 & -0.978543600 \\
\hline $\mathrm{H}$ & 3.901627700 & -4.336139400 & -0.087731500 \\
\hline $\mathrm{H}$ & 2.364165700 & -4.280084700 & 0.785447000 \\
\hline $\mathrm{H}$ & 0.512245500 & 6.566636700 & 0.170474000 \\
\hline \multicolumn{4}{|c|}{ Coord. 6} \\
\hline C & 0.050283000 & 4.146996700 & 0.104217600 \\
\hline C & 0.020008300 & 2.740908400 & 0.067483300 \\
\hline C & 1.223992200 & 1.959786900 & 0.056140000 \\
\hline C & 2.463081300 & 2.680245100 & 0.084051400 \\
\hline C & 2.487818800 & 4.090644700 & 0.120857800 \\
\hline C & 1.284811400 & 4.820660900 & 0.130880200 \\
\hline $\mathrm{H}$ & -0.871666900 & 4.701748600 & 0.111773300 \\
\hline $\mathrm{H}$ & -0.928822200 & 2.237390800 & 0.047361300 \\
\hline $\mathrm{H}$ & 3.395270200 & 2.150695800 & 0.077228800 \\
\hline $\mathrm{H}$ & 3.419342500 & 4.622988100 & 0.141598900 \\
\hline 0 & 1.376997900 & 6.172036600 & 0.166652300 \\
\hline C & 1.132039300 & 0.539286100 & 0.018576900 \\
\hline C & 2.183692500 & -0.359506200 & 0.002983800 \\
\hline $\mathrm{H}$ & 0.138758400 & 0.125837800 & 0.000526900 \\
\hline $\mathrm{H}$ & 3.208263900 & -0.043901600 & 0.018660800 \\
\hline C & 1.977544200 & -1.764289100 & -0.034921400 \\
\hline 0 & 0.831524600 & -2.325395300 & -0.057674200 \\
\hline 0 & 3.069281100 & -2.530630000 & -0.046978400 \\
\hline C & 2.904181200 & -3.933290600 & -0.084374800 \\
\hline $\mathrm{H}$ & 2.377608800 & -4.237125700 & -0.979103300 \\
\hline $\mathrm{H}$ & 3.900805100 & -4.346037500 & -0.088057800 \\
\hline $\mathrm{H}$ & 2.365235200 & -4.282727400 & 0.786006400 \\
\hline
\end{tabular}




\begin{tabular}{|c|c|c|c|}
\hline $\mathrm{H}$ & 0.516191200 & 6.565948100 & 0.170496600 \\
\hline C & 0.053150800 & 4.146852400 & 0.104224800 \\
\hline C & 0.020844300 & 2.747629700 & 0.067643800 \\
\hline C & 1.218407900 & 1.965941100 & 0.056248500 \\
\hline C & 2.454780400 & 2.683529800 & 0.084086400 \\
\hline $\mathrm{C}$ & 2.483696500 & 4.085292100 & 0.120709500 \\
\hline C & 1.285317700 & 4.818526800 & 0.130839000 \\
\hline $\mathrm{H}$ & -0.868160000 & 4.703334300 & 0.111821900 \\
\hline $\mathrm{H}$ & -0.929689200 & 2.246546300 & 0.047557100 \\
\hline $\mathrm{H}$ & 3.386423600 & 2.152187900 & 0.077220800 \\
\hline $\mathrm{H}$ & 3.416873300 & 4.615358200 & 0.141419800 \\
\hline 0 & 1.380318400 & 6.169977500 & 0.166644600 \\
\hline C & 1.123721400 & 0.541992300 & 0.018567100 \\
\hline C & 2.184334800 & -0.361485500 & 0.002915100 \\
\hline $\mathrm{H}$ & 0.129782600 & 0.130681300 & 0.000563800 \\
\hline $\mathrm{H}$ & 3.206777000 & -0.038856400 & 0.018749600 \\
\hline C & 1.995845900 & -1.755092500 & -0.034559500 \\
\hline 0 & 0.834555300 & -2.332100000 & -0.057781700 \\
\hline 0 & 3.079284300 & -2.535948100 & -0.047092100 \\
\hline C & 2.905609500 & -3.937125300 & -0.084454600 \\
\hline $\mathrm{H}$ & 2.378369300 & -4.239469700 & -0.979652200 \\
\hline $\mathrm{H}$ & 3.899778600 & -4.355554300 & -0.088374100 \\
\hline $\mathrm{H}$ & 2.366135100 & -4.285048000 & 0.786572900 \\
\hline $\mathrm{H}$ & 0.520084300 & 6.565101600 & 0.170515900 \\
\hline \multicolumn{4}{|c|}{ Coord. 7} \\
\hline C & 0.056031500 & 4.146543100 & 0.104228300 \\
\hline C & 0.021756000 & 2.754188100 & 0.067800800 \\
\hline C & 1.212928000 & 1.971995200 & 0.056354700 \\
\hline C & 2.446564000 & 2.686748700 & 0.084119900 \\
\hline C & 2.479575600 & 4.079862200 & 0.120559300 \\
\hline C & 1.285816400 & 4.816263300 & 0.130794900 \\
\hline $\mathrm{H}$ & -0.864653600 & 4.704727000 & 0.111866300 \\
\hline $\mathrm{H}$ & -0.930459300 & 2.255516200 & 0.047748700 \\
\hline $\mathrm{H}$ & 3.377670800 & 2.153636700 & 0.077211700 \\
\hline $\mathrm{H}$ & 3.414388700 & 4.607666200 & 0.141239300 \\
\hline 0 & 1.383594900 & 6.167785800 & 0.166634100 \\
\hline C & 1.115535800 & 0.544600900 & 0.018554700 \\
\hline C & 2.185152800 & -0.363505700 & 0.002845000 \\
\hline $\mathrm{H}$ & 0.120955200 & 0.135400200 & 0.000597500 \\
\hline $\mathrm{H}$ & 3.205408500 & -0.033837700 & 0.018837200 \\
\hline C & 2.014084000 & -1.745723800 & -0.034196200 \\
\hline 0 & 0.837686100 & -2.338862600 & -0.057891000 \\
\hline 0 & 3.089053500 & -2.540975900 & -0.047200800 \\
\hline
\end{tabular}




\begin{tabular}{|c|c|c|c|}
\hline C & 2.906855200 & -3.940622600 & -0.084525700 \\
\hline $\mathrm{H}$ & 2.378959100 & -4.241493800 & -0.980190300 \\
\hline $\mathrm{H}$ & 3.898549000 & -4.364690900 & -0.088680300 \\
\hline $\mathrm{H}$ & 2.366865700 & -4.287047700 & 0.787146300 \\
\hline $\mathrm{H}$ & 0.523924300 & 6.564098200 & 0.170532000 \\
\hline \multicolumn{4}{|c|}{ Coord. 8} \\
\hline C & 0.058925100 & 4.146070000 & 0.104228100 \\
\hline C & 0.022743100 & 2.760584400 & 0.067954100 \\
\hline C & 1.207552400 & 1.977949400 & 0.056458800 \\
\hline C & 2.438431900 & 2.689902200 & 0.084151900 \\
\hline C & 2.475456500 & 4.074355900 & 0.120407200 \\
\hline C & 1.286307500 & 4.813871500 & 0.130747900 \\
\hline $\mathrm{H}$ & -0.861148000 & 4.705927800 & 0.111906400 \\
\hline $\mathrm{H}$ & -0.931133100 & 2.264300700 & 0.047936200 \\
\hline $\mathrm{H}$ & 3.369011800 & 2.155042700 & 0.077201500 \\
\hline $\mathrm{H}$ & 3.411889200 & 4.599913000 & 0.141057200 \\
\hline 0 & 1.386827200 & 6.165462900 & 0.166620900 \\
\hline C & 1.107482800 & 0.547111900 & 0.018539800 \\
\hline C & 2.186147500 & -0.365565700 & 0.002773600 \\
\hline $\mathrm{H}$ & 0.112276400 & 0.139994300 & 0.000628000 \\
\hline $\mathrm{H}$ & 3.204158500 & -0.028844800 & 0.018923700 \\
\hline C & 2.032255700 & -1.736185200 & -0.033831600 \\
\hline 0 & 0.840917800 & -2.345685800 & -0.058002200 \\
\hline 0 & 3.098588300 & -2.545713700 & -0.047304300 \\
\hline C & 2.907918400 & -3.943783600 & -0.084588100 \\
\hline $\mathrm{H}$ & 2.379378200 & -4.243199300 & -0.980717800 \\
\hline $\mathrm{H}$ & 3.897117300 & -4.373448300 & -0.088976500 \\
\hline $\mathrm{H}$ & 2.367426800 & -4.288728100 & 0.787726700 \\
\hline $\mathrm{H}$ & 0.527710700 & 6.562939100 & 0.170544900 \\
\hline \multicolumn{4}{|c|}{ Coord.9 } \\
\hline C & 0.061831500 & 4.145434000 & 0.104224300 \\
\hline C & 0.023805200 & 2.766819300 & 0.068103900 \\
\hline C & 1.202281100 & 1.983804100 & 0.056560600 \\
\hline C & 2.430384300 & 2.692990600 & 0.084182500 \\
\hline C & 2.471339600 & 4.068774000 & 0.120253200 \\
\hline C & 1.286790900 & 4.811352200 & 0.130698100 \\
\hline $\mathrm{H}$ & -0.857643600 & 4.706937300 & 0.111942300 \\
\hline $\mathrm{H}$ & -0.931711100 & 2.272900200 & 0.048119500 \\
\hline $\mathrm{H}$ & 3.360446900 & 2.156406100 & 0.077190300 \\
\hline $\mathrm{H}$ & 3.409375200 & 4.592099500 & 0.140873500 \\
\hline 0 & 1.390015100 & 6.163009700 & 0.166605000 \\
\hline C & 1.099562800 & 0.549525400 & 0.018522400 \\
\hline C & 2.187319600 & -0.367664400 & 0.002700900 \\
\hline $\mathrm{H}$ & 0.103746500 & 0.144463400 & 0.000655300 \\
\hline
\end{tabular}




\begin{tabular}{|c|c|c|c|}
\hline $\mathrm{H}$ & 3.203027500 & -0.023876900 & 0.019009000 \\
\hline C & 2.050358600 & -1.726479200 & -0.033465900 \\
\hline 0 & 0.844251300 & -2.352572100 & -0.058115400 \\
\hline 0 & 3.107888600 & -2.550162100 & -0.047402500 \\
\hline C & 2.908799300 & -3.946609600 & -0.084641800 \\
\hline $\mathrm{H}$ & 2.379627000 & -4.244587700 & -0.981234700 \\
\hline $\mathrm{H}$ & 3.895484100 & -4.381827500 & -0.089262600 \\
\hline $\mathrm{H}$ & 2.367818600 & -4.290090400 & 0.788314000 \\
\hline $\mathrm{H}$ & 0.531443300 & 6.561625200 & 0.170554600 \\
\hline \multicolumn{4}{|c|}{ Coord.10 } \\
\hline C & 0.064750500 & 4.144636000 & 0.104216700 \\
\hline C & 0.024941700 & 2.772893400 & 0.068250100 \\
\hline C & 1.197113900 & 1.989559600 & 0.056660200 \\
\hline C & 2.422421000 & 2.696014000 & 0.084211600 \\
\hline C & 2.467225400 & 4.063117300 & 0.120097200 \\
\hline C & 1.287266600 & 4.808706500 & 0.130645400 \\
\hline $\mathrm{H}$ & -0.854140700 & 4.707756400 & 0.111974000 \\
\hline $\mathrm{H}$ & -0.932193800 & 2.281315200 & 0.048298800 \\
\hline $\mathrm{H}$ & 3.351976200 & 2.157727400 & 0.077177900 \\
\hline $\mathrm{H}$ & 3.406847200 & 4.584226900 & 0.140688400 \\
\hline 0 & 1.393158100 & 6.160427700 & 0.166586500 \\
\hline C & 1.091776300 & 0.551841700 & 0.018502400 \\
\hline C & 2.188670100 & -0.369800600 & 0.002627000 \\
\hline $\mathrm{H}$ & 0.095365900 & 0.148807600 & 0.000679400 \\
\hline $\mathrm{H}$ & 3.202015700 & -0.018933300 & 0.019093300 \\
\hline C & 2.068390300 & -1.716608100 & -0.033098900 \\
\hline 0 & 0.847687200 & -2.359524100 & -0.058230700 \\
\hline 0 & 3.116954300 & -2.554321400 & -0.047495500 \\
\hline C & 2.909498100 & -3.949101900 & -0.084686800 \\
\hline $\mathrm{H}$ & 2.379705400 & -4.245660400 & -0.981741100 \\
\hline $\mathrm{H}$ & 3.893650300 & -4.389829900 & -0.089538600 \\
\hline $\mathrm{H}$ & 2.368041000 & -4.291136300 & 0.788907900 \\
\hline $\mathrm{H}$ & 0.535121500 & 6.560157700 & 0.170561200 \\
\hline
\end{tabular}

LIIC path connecting the ${ }^{1} \mathrm{~mm}^{*}$ minima and the ${ }^{1} \mathrm{n} \pi^{*}$ minima of OMpCA-W1(10 points)

\section{Coord.1}

$\begin{array}{lrrr}\text { C } & -2.747656300 & 0.794856500 & -0.000610300 \\ \text { C } & -1.344310000 & 1.089556800 & 0.016855600 \\ \text { C } & -0.342090000 & 0.035243700 & -0.004105800 \\ \text { C } & -0.822770200 & -1.330089700 & -0.043514000 \\ \text { C } & -2.239674700 & -1.613231800 & -0.060772700 \\ \text { C } & -3.182368800 & -0.556007500 & -0.039320200 \\ H & -3.464664400 & 1.593356100 & 0.015553500 \\ H & -1.020130200 & 2.112215000 & 0.046126500 \\ H & -0.130597500 & -2.146788400 & -0.060343800\end{array}$


H

0

C

C

$\mathrm{H}$

$\mathrm{H}$

C

0

0

C

$\mathrm{H}$

$\mathrm{H}$

$\mathrm{H}$

$\mathrm{H}$

O

$\mathrm{H}$

$\mathrm{H}$

\section{Coord.2}

$\mathrm{C}$

C

C

C

C

C

$\mathrm{H}$

$\mathrm{H}$

H

$\mathrm{H}$

0

C

C

$\mathrm{H}$

$\mathrm{H}$

C

0

0

C

$\mathrm{H}$

H

$\mathrm{H}$

H

0

$\mathrm{H}$

$\mathrm{H}$

-2.591057900
-4.487755400
1.019690900
2.081190300
1.245569200
1.994620400
3.446554000
3.735030300
4.356416700
5.712911600
5.933185500
6.288446600
5.945307000
-5.045037800
-6.289341200
-6.858082600
-6.847600500

$-2.625732300$

$-0.089969700$

$-2.745664400$

$-1.350003800$

$-0.346802200$

$-0.820756900$

$-2.227297000$

$-3.174192000$

$-3.466445100$

$-1.031380000$

$-0.124539000$

$-2.573792000$

$-4.476488800$

1.018659800

2.080501900

1.244739700

1.980458300

3.444272800

3.754836900

4.359949100

5.712068900

5.982225500

6.293349700

5.887517100

$-5.040861900$

$-6.302472800$

$-6.877881500$

$-6.855727300$
$-0.891867100$

0.382562300

$-0.449190500$

1.435052000

$-1.517140600$

0.098583500

1.256832800

$-0.870080300$

$-0.473062000$

0.087749100

$-1.385792900$

0.134271100

$-0.118997000$

1.383073800

1.441587100

1.481624400

$-0.057205500$

0.014460400

$-0.000206600$

0.043833900

$-0.029027500$

0.023677100

0.056272000

0.004347000

0.024181200

0.921293100

0.004021600

$-0.838939800$

$-0.040625000$

$-0.009548600$

0.747082800

$-0.769822200$

0.803035400

$-0.002138600$

1.101883700

0.055133900

$-1.307877000$

$-1.596791100$

$-0.546668300$

1.598634200

2.126442700

$-2.121690600$

$-2.611005700$

$-0.892463700$

0.402251000

$-0.442471600$

1.454318100

$-1.509204800$

0.073622700

1.245585300

$-0.891901500$

$-0.488827200$

0.130304000

$-1.398163200$

0.060708900

$-0.125007800$

1.362506000

1.409963100

1.458626100
0.029308700

0.012776900

$-0.036911800$

$-0.068198300$

$-0.050765100$

0.011070300

0.066156600

$-0.050863800$

$-0.104998600$

$-0.082223200$

0.045148200

0.034719800

0.082001300

0.000381800

0.072588700

0.121878500

0.069629100

$-0.018007200$

0.825801200

$-0.012938000$

$-0.932204000$

$-0.067091900$

$-0.038342000$

0.714024900

$-0.802587500$ 


\begin{tabular}{|c|c|c|c|}
\hline \multicolumn{4}{|c|}{ Coord.3 } \\
\hline C & -2.742454700 & 0.810079400 & -0.002090700 \\
\hline C & -1.354710100 & 1.113026600 & 0.044102200 \\
\hline C & -0.350377300 & 0.074060800 & 0.032445200 \\
\hline C & -0.817474800 & -1.286594400 & -0.027960300 \\
\hline C & -2.213506700 & -1.581244400 & -0.074011200 \\
\hline C & -3.164697000 & -0.538332400 & -0.061056400 \\
\hline $\mathrm{H}$ & -3.467028200 & 1.602718900 & 0.007827000 \\
\hline $\mathrm{H}$ & -1.041804100 & 2.139427200 & 0.088852200 \\
\hline $\mathrm{H}$ & -0.117232900 & -2.097510000 & -0.038714900 \\
\hline $\mathrm{H}$ & -2.554920500 & -2.597144000 & -0.118766200 \\
\hline 0 & -4.463824200 & -0.893436000 & -0.106930600 \\
\hline C & 1.018338000 & 0.422044600 & 0.079579300 \\
\hline C & 2.081553100 & -0.434227100 & 0.073722200 \\
\hline $\mathrm{H}$ & 1.243642800 & 1.473825600 & 0.124271400 \\
\hline $\mathrm{H}$ & 1.969616700 & -1.499695700 & 0.033397800 \\
\hline C & 3.442010500 & 0.052855600 & 0.126166900 \\
\hline 0 & 3.772766900 & 1.238574700 & 0.192099900 \\
\hline 0 & 4.365232000 & -0.907539900 & 0.138510400 \\
\hline C & 5.705249300 & -0.505851000 & -0.065684900 \\
\hline $\mathrm{H}$ & 6.025581500 & 0.172090300 & 0.713155700 \\
\hline $\mathrm{H}$ & 6.294339300 & -1.409682400 & -0.035054400 \\
\hline $\mathrm{H}$ & 5.815676400 & -0.022171700 & -1.026457700 \\
\hline $\mathrm{H}$ & -5.034775900 & -0.131066800 & -0.093559200 \\
\hline 0 & -6.312619800 & 1.342472800 & -0.067318200 \\
\hline $\mathrm{H}$ & -6.895116100 & 1.378625800 & 0.680218500 \\
\hline $\mathrm{H}$ & -6.860133200 & 1.437124400 & -0.835878700 \\
\hline \multicolumn{4}{|c|}{ Coord.4 } \\
\hline C & -2.737765000 & 0.815713300 & -0.000188100 \\
\hline C & -1.358211300 & 1.122711100 & 0.061645800 \\
\hline C & -0.352558000 & 0.091807500 & 0.055229700 \\
\hline C & -0.812636000 & -1.266453500 & -0.016567100 \\
\hline C & -2.197993500 & -1.566814200 & -0.078250300 \\
\hline C & -3.153601400 & -0.531247600 & -0.070150000 \\
\hline $\mathrm{H}$ & -3.466158400 & 1.605321600 & 0.006168700 \\
\hline $\mathrm{H}$ & -1.051216700 & 2.150883600 & 0.114799700 \\
\hline $\mathrm{H}$ & -0.108393500 & -2.074456500 & -0.023878200 \\
\hline $\mathrm{H}$ & -2.534095300 & -2.584363000 & -0.131488500 \\
\hline 0 & -4.449496500 & -0.894895700 & -0.131423000 \\
\hline $\mathrm{C}$ & 1.018883100 & 0.441982100 & 0.118259100 \\
\hline C & 2.084819300 & -0.424072200 & 0.117315200 \\
\hline $\mathrm{H}$ & 1.242215400 & 1.493641400 & 0.171302800 \\
\hline $\mathrm{H}$ & 1.962927500 & -1.488309500 & 0.070405600 \\
\hline C & 3.440191700 & 0.037211100 & 0.185105300 \\
\hline
\end{tabular}




\begin{tabular}{|c|c|c|c|}
\hline 0 & 3.788650600 & 1.237021100 & 0.267307900 \\
\hline 0 & 4.373160000 & -0.915588900 & 0.211346300 \\
\hline C & 5.690993000 & -0.524449800 & -0.119392400 \\
\hline $\mathrm{H}$ & 6.060823400 & 0.212420000 & 0.580291300 \\
\hline $\mathrm{H}$ & 6.290633500 & -1.420092800 & -0.062898200 \\
\hline $\mathrm{H}$ & 5.726876400 & -0.116155700 & -1.120361300 \\
\hline $\mathrm{H}$ & -5.026417300 & -0.137184800 & -0.120027000 \\
\hline 0 & -6.319231300 & 1.323180800 & -0.096447800 \\
\hline $\mathrm{H}$ & -6.909240300 & 1.347837500 & 0.645652400 \\
\hline $\mathrm{H}$ & -6.860247900 & 1.417450400 & -0.869647500 \\
\hline \multicolumn{4}{|c|}{ Coord.5 } \\
\hline C & -2.731276000 & 0.819586000 & 0.003674600 \\
\hline C & -1.360239100 & 1.130589000 & 0.082074100 \\
\hline C & -0.353019300 & 0.108103300 & 0.081107700 \\
\hline C & -0.805876700 & -1.247716600 & -0.002970700 \\
\hline C & -2.180371100 & -1.553778900 & -0.081184800 \\
\hline C & -3.140560300 & -0.525727800 & -0.078156100 \\
\hline $\mathrm{H}$ & -3.463530400 & 1.606071600 & 0.006329000 \\
\hline $\mathrm{H}$ & -1.059390500 & 2.160449100 & 0.144297800 \\
\hline $\mathrm{H}$ & -0.097654800 & -2.052789000 & -0.006726400 \\
\hline $\mathrm{H}$ & -2.510881400 & -2.572931700 & -0.143596400 \\
\hline 0 & -4.433188300 & -0.896983200 & -0.155856300 \\
\hline C & 1.020492200 & 0.462123400 & 0.161247000 \\
\hline C & 2.090902100 & -0.411489800 & 0.165507800 \\
\hline $\mathrm{H}$ & 1.240369500 & 1.513856900 & 0.223289300 \\
\hline $\mathrm{H}$ & 1.961451200 & -1.474623300 & 0.111301400 \\
\hline C & 3.439333600 & 0.027889900 & 0.249491800 \\
\hline 0 & 3.802257700 & 1.242487900 & 0.347257400 \\
\hline 0 & 4.384833300 & -0.914202700 & 0.287943500 \\
\hline C & 5.667492600 & -0.545052500 & -0.178959100 \\
\hline $\mathrm{H}$ & 6.084032700 & 0.249821600 & 0.424770100 \\
\hline $\mathrm{H}$ & 6.281282800 & -1.429040500 & -0.096434600 \\
\hline $\mathrm{H}$ & 5.618413200 & -0.223083300 & -1.210741900 \\
\hline $\mathrm{H}$ & -5.015347500 & -0.143376200 & -0.146495600 \\
\hline 0 & -6.321625000 & 1.304894200 & -0.125670600 \\
\hline $\mathrm{H}$ & -6.919499500 & 1.318007900 & 0.610406300 \\
\hline $\mathrm{H}$ & -6.855449500 & 1.399948000 & -0.903758500 \\
\hline \multicolumn{4}{|c|}{ Coord. 6} \\
\hline C & -2.722669000 & 0.821286400 & 0.009393200 \\
\hline C & -1.360524500 & 1.136253900 & 0.105184300 \\
\hline C & -0.351415500 & 0.122640400 & 0.109581400 \\
\hline C & -0.796803800 & -1.230677200 & 0.012104600 \\
\hline C & -2.160230100 & -1.542452500 & -0.083445300 \\
\hline C & -3.125224000 & -0.522135800 & -0.085409300 \\
\hline
\end{tabular}


H

H

$\mathrm{H}$

$\mathrm{H}$

0

C

C

$\mathrm{H}$

$\mathrm{H}$

C

0

0

C

$\mathrm{H}$

$\mathrm{H}$

$\mathrm{H}$

$\mathrm{H}$

O

$\mathrm{H}$

$\mathrm{H}$

\section{Coord.7}

C

C

C

C

C

C

$\mathrm{H}$

H

$\mathrm{H}$

$\mathrm{H}$

0

C

C

$\mathrm{H}$

$\mathrm{H}$

C

0

0

C

H

$\mathrm{H}$

$\mathrm{H}$

$\mathrm{H}$

-3.458849900
-1.066105500
-0.084612700
-2.484811900
-4.414592900
1.023360500
2.100472900
1.237963800
1.966399800
3.440001400
3.813276800
4.401463400
5.632855000
6.089939200
6.265354400
5.488515200
-5.001125000
-6.319074900
-6.924983700
-6.845197600

1.604529400

2.167695800

$-2.032791000$

$-2.563148900$

$-0.899858100$

0.482550900

$-0.395838600$

1.534580800

$-1.458095500$

0.026314200

1.256806300

$-0.901140700$

$-0.568203300$

0.281844900

$-1.436078100$

$-0.344628700$

$-0.149657700$

1.287924000

1.289714000

1.384914700

$-2.711719400$

$-1.358880100$

$-0.347456900$

$-0.785067900$

$-2.137217300$

$-3.107325000$

$-3.451937400$

$-1.071234600$

$-0.068885800$

$-2.455464400$

$-4.393502600$

1.027615800

2.114164700

1.234763000

1.978983800

3.442716200

3.821283200

4.424185600

5.585528400

6.072444600

6.242206000

5.337277800

$-4.983419000$
0.820387800

1.139288400

0.135122400

$-1.215603800$

$-1.533128900$

$-0.520834800$

1.600224800

2. 172173200

$-2.014707300$

$-2.555282700$

$-0.903674500$

0.503381800

$-0.376371800$

1.555935500

$-1.438062900$

0.034017300

1.281910400

$-0.873907500$

$-0.594574400$

0.304866400

$-1.440719200$

$-0.481997800$

$-0.156046700$
0.008439700

0.177322000

0.011773900

$-0.155892600$

$-0.180493700$

0.208009700

0.217610300

0.279838600

0.155277800

0.318600600

0.430928000

0.367335800

$-0.243242500$

0.245466700

$-0.134837800$

$-1.292319200$

$-0.172964900$

$-0.154882000$

0.574707800

$-0.937955800$

0.016641700

0.130411200

0.139586200

0.027304400

$-0.086157500$

$-0.092534000$

0.012572500

0.213542200

0.029863400

$-0.169726000$

$-0.205756500$

0.257318200

0.272071600

0.339912700

0.200619800

0.390727400

0.516431400

0.447582100

$-0.309922200$

0.043357100

$-0.176365300$

$-1.357696100$

$-0.199435200$ 


\begin{tabular}{|c|c|c|c|}
\hline 0 & -6.310962100 & 1.272593600 & -0.183924300 \\
\hline $\mathrm{H}$ & -6.924744800 & 1.263687500 & 0.538990300 \\
\hline $\mathrm{H}$ & -6.829232800 & 1.372505600 & -0.971840300 \\
\hline \multicolumn{4}{|c|}{ Coord.8 } \\
\hline C & -2.698414500 & 0.816529200 & 0.024905400 \\
\hline C & -1.355307800 & 1.139350800 & 0.156871900 \\
\hline C & -0.341009400 & 0.145356400 & 0.169474700 \\
\hline C & -0.770447800 & -1.202636900 & 0.040555600 \\
\hline C & -2.111125400 & -1.525982700 & -0.091043900 \\
\hline C & -3.086779900 & -0.522101500 & -0.100482100 \\
\hline $\mathrm{H}$ & -3.442859700 & 1.592726200 & 0.018822200 \\
\hline $\mathrm{H}$ & -1.074859900 & 2.173487000 & 0.252425200 \\
\hline $\mathrm{H}$ & -0.050183500 & -1.998625700 & 0.044851000 \\
\hline $\mathrm{H}$ & -2.422544300 & -2.549461700 & -0.187150400 \\
\hline 0 & -4.369904900 & -0.908537200 & -0.232271300 \\
\hline C & 1.033235500 & 0.524794200 & 0.307247500 \\
\hline C & 2.132417600 & -0.352272800 & 0.326413700 \\
\hline $\mathrm{H}$ & 1.230397500 & 1.578058600 & 0.401892000 \\
\hline $\mathrm{H}$ & 2.000179000 & -1.413741600 & 0.244575900 \\
\hline C & 3.447819500 & 0.052456400 & 0.463147500 \\
\hline 0 & 3.825712000 & 1.319564900 & 0.601063600 \\
\hline 0 & 4.453775500 & -0.830024900 & 0.525662900 \\
\hline C & 5.524845200 & -0.624986900 & -0.375448000 \\
\hline $\mathrm{H}$ & 6.025615200 & 0.313951000 & -0.177774300 \\
\hline $\mathrm{H}$ & 6.211800600 & -1.442562100 & -0.218367200 \\
\hline $\mathrm{H}$ & 5.167594600 & -0.635551000 & -1.397780200 \\
\hline $\mathrm{H}$ & -4.962148600 & -0.162558800 & -0.225906600 \\
\hline 0 & -6.296979200 & 1.259173700 & -0.212586500 \\
\hline $\mathrm{H}$ & -6.917976800 & 1.240747400 & 0.503939000 \\
\hline $\mathrm{H}$ & -6.807822700 & 1.362602800 & -1.004867600 \\
\hline \multicolumn{4}{|c|}{ Coord.9 } \\
\hline C & -2.683067400 & 0.809534700 & 0.033560900 \\
\hline C & -1.350103100 & 1.136302700 & 0.183487600 \\
\hline C & -0.332193600 & 0.153387600 & 0.197101100 \\
\hline C & -0.752928000 & -1.191634100 & 0.049074600 \\
\hline C & -2.081968700 & -1.520917000 & -0.100457200 \\
\hline C & -3.063777300 & -0.525996100 & -0.110526300 \\
\hline $\mathrm{H}$ & -3.432058900 & 1.581740500 & 0.027419500 \\
\hline $\mathrm{H}$ & -1.077391200 & 2.171412600 & 0.293418300 \\
\hline $\mathrm{H}$ & -0.028363100 & -1.984298000 & 0.053088600 \\
\hline $\mathrm{H}$ & -2.385949000 & -2.545509600 & -0.211020100 \\
\hline 0 & -4.344055000 & -0.914434700 & -0.260894300 \\
\hline C & 1.039971300 & 0.547066100 & 0.355311400 \\
\hline C & 2.155295200 & -0.322717800 & 0.377310700 \\
\hline
\end{tabular}




\begin{tabular}{|c|c|c|c|}
\hline $\mathrm{H}$ & 1.224348400 & 1.601107100 & 0.463789200 \\
\hline $\mathrm{H}$ & 2.030429300 & -1.384241500 & 0.283355700 \\
\hline C & 3.455320000 & 0.082737000 & 0.532248400 \\
\hline 0 & 3.825878700 & 1.370978400 & 0.681547100 \\
\hline 0 & 4.490305500 & -0.767469800 & 0.597541100 \\
\hline C & 5.451548400 & -0.660431700 & -0.435205100 \\
\hline $\mathrm{H}$ & 5.945047000 & 0.302845400 & -0.410914300 \\
\hline $\mathrm{H}$ & 6.174963100 & -1.441489900 & -0.257459300 \\
\hline $\mathrm{H}$ & 4.985757700 & -0.804355400 & -1.402686300 \\
\hline $\mathrm{H}$ & -4.937612900 & -0.169202700 & -0.252379200 \\
\hline 0 & -6.277353600 & 1.247787900 & -0.240620200 \\
\hline $\mathrm{H}$ & -6.904226300 & 1.221673500 & 0.470501500 \\
\hline $\mathrm{H}$ & -6.782011200 & 1.354665100 & -1.036373000 \\
\hline \multicolumn{4}{|c|}{ Coord.10 } \\
\hline C & -2.666383000 & 0.799561400 & 0.041986100 \\
\hline C & -1.343920300 & 1.130365900 & 0.209154900 \\
\hline C & -0.321455300 & 0.159669200 & 0.220004600 \\
\hline C & -0.732747800 & -1.181970000 & 0.049502500 \\
\hline C & -2.050022600 & -1.517366200 & -0.117308200 \\
\hline C & -3.038818200 & -0.532194800 & -0.124196000 \\
\hline $\mathrm{H}$ & -3.420429600 & 1.567234300 & 0.038834400 \\
\hline $\mathrm{H}$ & -1.079643900 & 2.166029800 & 0.336160900 \\
\hline $\mathrm{H}$ & -0.003463100 & -1.970906300 & 0.050110700 \\
\hline $\mathrm{H}$ & -2.345797000 & -2.542723100 & -0.244960100 \\
\hline 0 & -4.316489200 & -0.921152100 & -0.292696600 \\
\hline C & 1.047310200 & 0.570616100 & 0.398724100 \\
\hline C & 2.182312400 & -0.286982700 & 0.420821700 \\
\hline $\mathrm{H}$ & 1.215994100 & 1.625255900 & 0.523575700 \\
\hline $\mathrm{H}$ & 2.069318900 & -1.348619500 & 0.312286500 \\
\hline C & 3.464776900 & 0.125242900 & 0.593836100 \\
\hline 0 & 3.821115600 & 1.436321200 & 0.754444800 \\
\hline 0 & 4.532827600 & -0.685302900 & 0.658418300 \\
\hline C & 5.368123800 & -0.702059900 & -0.483898700 \\
\hline $\mathrm{H}$ & 5.829330300 & 0.264223500 & -0.645834800 \\
\hline $\mathrm{H}$ & 6.133465800 & -1.437929400 & -0.289833500 \\
\hline $\mathrm{H}$ & 4.801386000 & -0.985678300 & -1.363032700 \\
\hline $\mathrm{H}$ & -4.910572500 & -0.175974600 & -0.278852900 \\
\hline 0 & -6.253039700 & 1.238302900 & -0.267775100 \\
\hline $\mathrm{H}$ & -6.883588800 & 1.207034600 & 0.439842000 \\
\hline $\mathrm{H}$ & -6.753806000 & 1.347586000 & -1.065621500 \\
\hline
\end{tabular}

LIIC path connecting the ${ }^{1} \mathrm{~mm}^{*}$ minima and the ${ }^{1} \mathrm{n} \pi^{*}$ minima of OMpCA-W2(10 points) Coord.1

$\begin{array}{llll}\text { C } & -2.910786800 & 0.847882500 & -0.016567900 \\ \text { C } & -1.485130000 & 1.024347300 & -0.013460100\end{array}$




\begin{tabular}{|c|c|c|c|}
\hline C & -0.584659700 & -0.122370600 & 0.010414600 \\
\hline C & -1.175438500 & -1.447327400 & 0.033932600 \\
\hline C & -2.615050700 & -1.605668800 & 0.033484800 \\
\hline C & -3.456201500 & -0.463447100 & 0.007176600 \\
\hline $\mathrm{H}$ & -3.554634900 & 1.707900700 & -0.038767400 \\
\hline $\mathrm{H}$ & -1.067399200 & 2.012195300 & -0.039383600 \\
\hline $\mathrm{H}$ & -0.555367200 & -2.319712100 & 0.056807600 \\
\hline $\mathrm{H}$ & -3.057996500 & -2.581507000 & 0.051599400 \\
\hline 0 & -4.792746900 & -0.687382400 & 0.007038400 \\
\hline C & 0.814583400 & 0.118278500 & 0.006829500 \\
\hline C & 1.797286000 & -0.785564600 & 0.006443300 \\
\hline $\mathrm{H}$ & 1.103535700 & 1.154342300 & 0.003216800 \\
\hline $\mathrm{H}$ & 1.630510600 & -1.844591500 & 0.007679900 \\
\hline C & 3.205508200 & -0.351076500 & -0.002863700 \\
\hline 0 & 3.596629400 & 0.782043600 & -0.006093800 \\
\hline 0 & 4.029947100 & -1.383918100 & -0.006930600 \\
\hline C & 5.418970300 & -1.107570700 & -0.023271100 \\
\hline $\mathrm{H}$ & 5.704399700 & -0.547175500 & 0.855122800 \\
\hline $\mathrm{H}$ & 5.908994700 & -2.068422100 & -0.029767200 \\
\hline $\mathrm{H}$ & 5.683208000 & -0.545606900 & -0.907164800 \\
\hline 0 & 2.161062200 & 3.386706900 & -0.018035000 \\
\hline $\mathrm{H}$ & 2.750760300 & 2.649837200 & -0.146408200 \\
\hline $\mathrm{H}$ & 2.374516500 & 3.723125800 & 0.841370900 \\
\hline $\mathrm{H}$ & -5.269297700 & 0.130136000 & -0.019847700 \\
\hline \multicolumn{4}{|c|}{ Coord.2 } \\
\hline C & -2.907908400 & 0.843975900 & -0.040625300 \\
\hline C & -1.489310900 & 1.019516800 & -0.027665200 \\
\hline C & -0.590660800 & -0.121124300 & 0.016076600 \\
\hline C & -1.181522500 & -1.441248600 & 0.048834000 \\
\hline C & -2.612069100 & -1.601223100 & 0.037982800 \\
\hline C & -3.453934300 & -0.463418500 & -0.007492600 \\
\hline $\mathrm{H}$ & -3.551750900 & 1.703942700 & -0.077171500 \\
\hline $\mathrm{H}$ & -1.072791200 & 2.008061200 & -0.059945700 \\
\hline $\mathrm{H}$ & -0.561530300 & -2.313726100 & 0.086119200 \\
\hline $\mathrm{H}$ & -3.054097300 & -2.577668100 & 0.062946600 \\
\hline 0 & -4.790375600 & -0.688032600 & -0.016491100 \\
\hline C & 0.811106500 & 0.120068700 & 0.022027600 \\
\hline C & 1.798270800 & -0.794310900 & 0.040437000 \\
\hline $\mathrm{H}$ & 1.101930000 & 1.155394100 & 0.009109700 \\
\hline $\mathrm{H}$ & 1.622009900 & -1.851836200 & 0.053409800 \\
\hline C & 3.199213000 & -0.381063000 & 0.039485300 \\
\hline 0 & 3.602675400 & 0.769027500 & 0.032475900 \\
\hline 0 & 4.040520300 & -1.402524400 & 0.066970600 \\
\hline C & 5.419632900 & -1.107789100 & -0.065069600 \\
\hline
\end{tabular}


H

$\mathrm{H}$

$\mathrm{H}$

0

$\mathrm{H}$

$\mathrm{H}$

$\mathrm{H}$

\section{Coord.3}

C

C

C

C

C

C

$\mathrm{H}$

$\mathrm{H}$

$\mathrm{H}$

$\mathrm{H}$

0

C

C

$\mathrm{H}$

$\mathrm{H}$

C

0

0

C

$\mathrm{H}$

H

$\mathrm{H}$

0

H

$\mathrm{H}$

$\mathrm{H}$

Coord.4

C

C

C

C

C

C

$\mathrm{H}$

$\mathrm{H}$

H
5.752744300

5.928362200

5.613101600

2. 163328000

2.773289900

2.361698100

$-5.266729300$

$-2.904235100$

$-1.492998200$

$-0.595138600$

$-1.184705900$

$-2.605893500$

$-3.449515600$

$-3.548867600$

$-1.078716300$

$-0.563979200$

$-3.045985800$

$-4.785504800$

0.808780900

1.801176000

1.100417400

1.616489400

3.194023200

3.609143300

4.052934800

5.413198000

5.793845300

5.941673300

5.528403300

2. 166544000

2.797954200

2.348599700

$-5.262440000$

$-2.899511700$

$-1.496027800$

$-0.597674200$

$-1.184210800$

$-2.595649500$

$-3.442332200$

$-3.545939500$

$-1.085295900$

$-0.561722000$
$-0.475403100$

$-2.058266700$

$-0.614629600$

3.409140700

2.695205000

3.714255000

0.129131600

0.838363100

1.014695900

$-0.118582400$

$-1.434398400$

$-1.597720500$

$-0.465564000$

1.697345800

2.004295300

$-2.306055500$

$-2.575159600$

$-0.692477900$

0.124857500

$-0.798732100$

1.159637700

$-1.854704500$

$-0.404792500$

0.762484000

$-1.412794300$

$-1.113839600$

$-0.409661000$

$-2.050858300$

$-0.702684000$

3.434718400

2.746436400

3.707023700

0.123621800

0.830679100

1.009951400

$-0.114361200$

$-1.426554900$

$-1.595367200$

$-0.470398100$

1.687511100

2.001074000

$-2.296252400$
0.745398300

$-0.029412700$

$-1.006860100$

0.017408100

$-0.133319800$

0.893052700

$-0.055124700$

$-0.066659900$

$-0.042735600$

0.022882700

0.065670700

0.043372400

$-0.023306200$

$-0.119164800$

$-0.082112500$

0.118969100

0.075878800

$-0.042225100$

0.039528800

0.079085200

0.016477700

0.104607600

0.087741300

0.075104000

0.147286000

$-0.111171200$

0.615202400

$-0.031941200$

$-1.104145300$

0.053323300

$-0.119160600$

0.944042000

$-0.093993200$

$-0.094377300$

$-0.058282300$

0.030881200

0.084041800

0.049175500

$-0.040412300$

$-0.164211400$

$-0.105175700$

0.154710000 
H

0

C

C

$\mathrm{H}$

$\mathrm{H}$

C

0

0

C

$\mathrm{H}$

$\mathrm{H}$

$\mathrm{H}$

0

$\mathrm{H}$

$\mathrm{H}$

$\mathrm{H}$

\section{Coord.5}

C

C

C

C

C

C

$\mathrm{H}$

$\mathrm{H}$

$\mathrm{H}$

$\mathrm{H}$

0

C

C

H

$\mathrm{H}$

C

0

0

C

$\mathrm{H}$

$\mathrm{H}$

$\mathrm{H}$

O

$\mathrm{H}$

$\mathrm{H}$

$\mathrm{H}$

-3.032506900
-4.777411000
0.807906900
1.806547700
1.099012300
1.614779100
3.190408300
3.616167100
4.067848100
5.398002400
5.825045100
5.947617300
5.426361000
2.170337600
2.824390900
2.334971800
-5.255911400

$-2.574302700$

0.089599500

$-0.701667300$

$-0.070434600$

0.059520300

0.122372600

$-0.797591700$

1.167995800

$-1.852021300$

$-0.420593900$

0.764191800

$-1.412770000$

$-1.127548000$

$-0.354193500$

$-2.047461500$

$-0.812558400$

3.464512100

2.804974900

3.702224700

0.112484500

0.025850700

0.160891400

0.142101300

0.121947300

0.233572300

$-0.161474900$

0.461888000

$-0.037648000$

$-1.195404000$

0.089691100

$-0.103962700$

0.994184900

$-0.136488400$

$-2.893429000$

0.820574000

$-0.123109800$

$-1.498199900$

1.005399100

$-0.108029500$

$-1.417492300$

$-0.073745200$

0.039915500

0.103177800

$-1.594395200$

0.054746900

$-0.058756600$

$-0.211116000$

$-0.128120000$

0.192041100

0.103016900

$-0.101011000$

0.081798600

0.169515600

0.037531400

0.220929000

0.201804600

0.172423500

0.324178200

$-0.215118700$

0.284123900

$-0.046468200$

$-1.274968400$

0.126470000

$-0.087959900$

1.043274000

$-0.182017200$ 


\begin{tabular}{|c|c|c|c|}
\hline \multicolumn{4}{|c|}{ Coord. 6} \\
\hline C & -2.885690700 & 0.807790200 & -0.151785800 \\
\hline C & -1.499333500 & 1.001202700 & -0.088393600 \\
\hline C & -0.594917100 & -0.099173000 & 0.049580600 \\
\hline C & -1.168439800 & -1.407027500 & 0.121887600 \\
\hline C & -2.558735700 & -1.595031800 & 0.059249100 \\
\hline C & -3.416709400 & -0.490235000 & -0.078061300 \\
\hline $\mathrm{H}$ & -3.539747800 & 1.655929600 & -0.257954200 \\
\hline $\mathrm{H}$ & -1.101037700 & 1.997350600 & -0.149610200 \\
\hline $\mathrm{H}$ & -0.538298400 & -2.268532200 & 0.228916900 \\
\hline $\mathrm{H}$ & -2.983852700 & -2.579069400 & 0.114706300 \\
\hline 0 & -4.748047200 & -0.738252300 & -0.133411900 \\
\hline C & 0.811928700 & 0.166074600 & 0.105688800 \\
\hline C & 1.827320500 & -0.773323500 & 0.218816100 \\
\hline $\mathrm{H}$ & 1.096553700 & 1.201225200 & 0.051527000 \\
\hline $\mathrm{H}$ & 1.626793100 & -1.825144000 & 0.282283000 \\
\hline C & 3.190197500 & -0.421279000 & 0.264941000 \\
\hline 0 & 3.632487500 & 0.800043000 & 0.225065000 \\
\hline 0 & 4.108374100 & -1.373771100 & 0.416026900 \\
\hline C & 5.333431600 & -1.185789800 & -0.270334000 \\
\hline $\mathrm{H}$ & 5.840614500 & -0.297845700 & 0.082704100 \\
\hline $\mathrm{H}$ & 5.931449900 & -2.058176200 & -0.057890300 \\
\hline $\mathrm{H}$ & 5.161340100 & -1.104000800 & -1.335300800 \\
\hline 0 & 2.177498500 & 3.541129800 & 0.163657300 \\
\hline $\mathrm{H}$ & 2.880089700 & 2.950451700 & -0.071559200 \\
\hline $\mathrm{H}$ & 2.305564400 & 3.702195300 & 1.091079700 \\
\hline $\mathrm{H}$ & -5.233631400 & 0.068713300 & -0.229272000 \\
\hline \multicolumn{4}{|c|}{ Coord.7 } \\
\hline C & -2.876104600 & 0.792243300 & -0.178959400 \\
\hline C & -1.499338000 & 0.997560000 & -0.101349100 \\
\hline C & -0.588724100 & -0.087478100 & 0.059215800 \\
\hline C & -1.151370000 & -1.395070700 & 0.138564800 \\
\hline C & -2.530031600 & -1.597454900 & 0.061646500 \\
\hline C & -3.396887000 & -0.506015900 & -0.097843100 \\
\hline $\mathrm{H}$ & -3.536535800 & 1.633561600 & -0.302108600 \\
\hline $\mathrm{H}$ & -1.110660000 & 1.997519900 & -0.168018100 \\
\hline $\mathrm{H}$ & -0.514785200 & -2.250080600 & 0.262558500 \\
\hline $\mathrm{H}$ & -2.945925400 & -2.585421600 & 0.122907900 \\
\hline 0 & -4.725116100 & -0.767191800 & -0.166680800 \\
\hline C & 0.817423600 & 0.191586000 & 0.130036000 \\
\hline C & 1.843985500 & -0.748050900 & 0.267643100 \\
\hline $\mathrm{H}$ & 1.095383300 & 1.227936700 & 0.067532300 \\
\hline $\mathrm{H}$ & 1.642492300 & -1.799129800 & 0.341411100 \\
\hline C & 3.194751500 & -0.403308200 & 0.328420500 \\
\hline
\end{tabular}




\begin{tabular}{|c|c|c|c|}
\hline 0 & 3.642038200 & 0.837354400 & 0.277502600 \\
\hline 0 & 4.135608100 & -1.331720900 & 0.504604000 \\
\hline C & 5.280378000 & -1.233484900 & -0.324419800 \\
\hline $\mathrm{H}$ & 5.814097800 & -0.310645200 & -0.138756700 \\
\hline $\mathrm{H}$ & 5.906771400 & -2.074481200 & -0.070932900 \\
\hline $\mathrm{H}$ & 4.996810500 & -1.283894900 & -1.367598400 \\
\hline 0 & 2.179759500 & 3.589536200 & 0.201345000 \\
\hline $\mathrm{H}$ & 2.907665100 & 3.040307900 & -0.055272200 \\
\hline $\mathrm{H}$ & 2.290261300 & 3.706927300 & 1.137363200 \\
\hline $\mathrm{H}$ & -5.216745900 & 0.034351200 & -0.276256100 \\
\hline \multicolumn{4}{|c|}{ Coord.8 } \\
\hline C & -2.864681000 & 0.774080800 & -0.202911100 \\
\hline C & -1.498290500 & 0.994670500 & -0.111629000 \\
\hline C & -0.579017800 & -0.072816900 & 0.067951800 \\
\hline C & -1.127386400 & -1.381663200 & 0.151264400 \\
\hline C & -2.493584800 & -1.601741200 & 0.060721100 \\
\hline C & -3.371846600 & -0.525826700 & -0.117481200 \\
\hline $\mathrm{H}$ & -3.533507800 & 1.606943000 & -0.340429800 \\
\hline $\mathrm{H}$ & -1.121910100 & 1.999474400 & -0.181484300 \\
\hline $\mathrm{H}$ & -0.482290100 & -2.228602300 & 0.289591900 \\
\hline $\mathrm{H}$ & -2.897614400 & -2.594728400 & 0.125560800 \\
\hline 0 & -4.695997600 & -0.803563600 & -0.199563900 \\
\hline C & 0.825388500 & 0.223730200 & 0.153301200 \\
\hline C & 1.865401100 & -0.713445600 & 0.312594600 \\
\hline $\mathrm{H}$ & 1.094001300 & 1.262037500 & 0.084995100 \\
\hline $\mathrm{H}$ & 1.665615100 & -1.764411900 & 0.393871800 \\
\hline C & 3.202989900 & -0.372411400 & 0.388169800 \\
\hline 0 & 3.652575600 & 0.888516000 & 0.326632800 \\
\hline 0 & 4.168323300 & -1.274016800 & 0.584221600 \\
\hline C & 5.212006300 & -1.294367600 & -0.373888700 \\
\hline $\mathrm{H}$ & 5.757122200 & -0.359244400 & -0.373487300 \\
\hline $\mathrm{H}$ & 5.870250500 & -2.098597500 & -0.084214400 \\
\hline $\mathrm{H}$ & 4.813146400 & -1.481121600 & -1.362787600 \\
\hline 0 & 2.180658800 & 3.644834000 & 0.239757000 \\
\hline $\mathrm{H}$ & 2.933767100 & 3.142743300 & -0.039601600 \\
\hline $\mathrm{H}$ & 2.275729900 & 3.713615100 & 1.181853500 \\
\hline $\mathrm{H}$ & -5.195646300 & -0.008630900 & -0.320452400 \\
\hline \multicolumn{4}{|c|}{ Coord.9 } \\
\hline C & -2.851715000 & 0.753691700 & -0.221794500 \\
\hline C & -1.496497700 & 0.992686200 & -0.118193700 \\
\hline C & -0.565964200 & -0.055309600 & 0.074806300 \\
\hline C & -1.096478900 & -1.366983600 & 0.157838500 \\
\hline C & -2.449356800 & -1.607814200 & 0.055103900 \\
\hline C & -3.341701300 & -0.549344900 & -0.136323800 \\
\hline
\end{tabular}


$\mathrm{H}$

H

$\mathrm{H}$

$\mathrm{H}$

0

C

C

$\mathrm{H}$

$\mathrm{H}$

C

0

0

C

$\mathrm{H}$

$\mathrm{H}$

$\mathrm{H}$

0

$\mathrm{H}$

$\mathrm{H}$

$\mathrm{H}$

\section{Coord.10}

C

C

C

C

C

C

$\mathrm{H}$

H

$\mathrm{H}$

$\mathrm{H}$

0

C

C

$\mathrm{H}$

$\mathrm{H}$

C

0

0

C

H

$\mathrm{H}$

$\mathrm{H}$

0

-3.531103400
-1.135264000
-0.440653900
-2.838714400
-4.660765000
0.835613000
1.891586900
1.092040800
1.696339700
3.215042900
3.663995000
4.206729800
5.128574100
5.664980600
5.822495000
4.615232300
2.180236700
2.957444500
2.263646200
-5.170540400

1.576654300

$-0.369486900$

2.003403100

$-2.204467900$

$-2.606953300$

$-0.846870200$

0.262220300

$-0.670011900$

1.303318900

$-1.721661100$

$-0.329343000$

0.952897400

$-1.201686700$

$-1.367599100$

$-0.447959200$

$-2.129833600$

$-1.688213200$

3.706183900

3.257663800

3.720366700

$-0.059579800$

$-2.837834400$

$-1.494528900$

$-0.550154600$

$-1.059301200$

$-2.398043300$

$-3.307133800$

$-3.529967500$

$-1.151276700$

$-0.390511300$

$-2.769933700$

$-4.620141100$

0.847547600

1.922092400

1.088980300

1.734193400

3.230665400

3.676016800

4.250509300

5.031858200

5.534840400

5.765378100

4.410898500

2.178991200
0.731664300

0.991651800

$-0.035350000$

$-1.351315000$

$-1.615398200$

$-0.575888900$

1.543594900

2.009267300

$-2.178267700$

$-2.621738000$

$-0.895949500$

0.306166900

$-0.619071200$

1.350875600

$-1.672286500$

$-0.275992100$

1.028669700

$-1.117021300$

$-1.451046800$

$-0.578483400$

$-2.166501400$

$-1.895530600$

3.771878500
$-0.187994700$

0.306284900

0.120361000

$-0.230705000$

0.173749600

0.349813100

0.103260400

0.434698500

0.439528800

0.368947300

0.648518700

$-0.414766100$

$-0.611701600$

$-0.096116700$

$-1.312701700$

0.279254900

$-0.024898600$

1.224179400

$-0.359105800$

$-0.233791300$

$-0.119986700$

0.078804600

0.156093500

0.043317000

$-0.153801500$

$-0.385839500$

$-0.185450800$

0.308821100

0.104830500

$-0.258866700$

0.189683700

0.375374300

0.121759000

0.458844300

0.477741200

0.400941900

0.691072800

$-0.442970400$

$-0.841143100$

$-0.105003900$

$-1.211188900$

0.320312700 


\begin{tabular}{|c|c|c|c|}
\hline $\mathrm{H}$ & 2.977898900 & 3.384000200 & -0.011214300 \\
\hline $\mathrm{H}$ & 2.256315400 & 3.724624300 & 1.263760600 \\
\hline $\mathrm{H}$ & -5.142157100 & -0.117098200 & -0.389543900 \\
\hline \multicolumn{4}{|c|}{ Cl-WO } \\
\hline C & -0.569720000 & 3.265295000 & 1.808566000 \\
\hline C & -0.299789000 & 2.187201000 & 0.959166000 \\
\hline C & 0.941725000 & 1.554045000 & 0.957516000 \\
\hline C & 1.928948000 & 2.044995000 & 1.857493000 \\
\hline C & 1.668893000 & 3.091588000 & 2.684305000 \\
\hline C & 0.403337000 & 3.713074000 & 2.663649000 \\
\hline $\mathrm{H}$ & -1.537612000 & 3.736720000 & 1.787727000 \\
\hline $\mathrm{H}$ & -1.071845000 & 1.841894000 & 0.294784000 \\
\hline $\mathrm{H}$ & 2.895248000 & 1.577564000 & 1.876826000 \\
\hline $\mathrm{H}$ & 2.410976000 & 3.467159000 & 3.363985000 \\
\hline 0 & 0.227906000 & 4.745611000 & 3.512438000 \\
\hline C & 1.193025000 & 0.442043000 & 0.071509000 \\
\hline C & 2.492215000 & -0.232662000 & -0.096566000 \\
\hline $\mathrm{H}$ & 0.414750000 & 0.238550000 & -0.646943000 \\
\hline $\mathrm{H}$ & 3.270188000 & 0.027212000 & -0.785843000 \\
\hline C & 2.403807000 & -1.323692000 & 0.715487000 \\
\hline 0 & 1.280948000 & -1.429255000 & 1.327370000 \\
\hline 0 & 3.362031000 & -2.233694000 & 0.899915000 \\
\hline C & 3.071179000 & -3.331393000 & 1.735773000 \\
\hline $\mathrm{H}$ & 2.226790000 & -3.894857000 & 1.360218000 \\
\hline $\mathrm{H}$ & 3.954997000 & -3.950733000 & 1.728323000 \\
\hline $\mathrm{H}$ & 2.860547000 & -3.007502000 & 2.746482000 \\
\hline $\mathrm{H}$ & -0.648234000 & 5.097767000 & 3.436667000 \\
\hline \multicolumn{4}{|c|}{ Cl-W1 } \\
\hline C & -0.266261000 & 3.143487000 & 1.814694000 \\
\hline C & -0.201664000 & 2.028349000 & 1.012340000 \\
\hline C & 0.996038000 & 1.322682000 & 0.828174000 \\
\hline C & 2.141419000 & 1.792656000 & 1.494090000 \\
\hline C & 2.091697000 & 2.904093000 & 2.297608000 \\
\hline C & 0.885980000 & 3.587473000 & 2.465820000 \\
\hline $\mathrm{H}$ & -1.191152000 & 3.675096000 & 1.946396000 \\
\hline $\mathrm{H}$ & -1.092575000 & 1.687220000 & 0.514956000 \\
\hline $\mathrm{H}$ & 3.067028000 & 1.265764000 & 1.361877000 \\
\hline $\mathrm{H}$ & 2.964466000 & 3.267587000 & 2.806679000 \\
\hline 0 & 0.890651000 & 4.661257000 & 3.255059000 \\
\hline C & 1.027660000 & 0.165792000 & -0.016761000 \\
\hline C & 2.213002000 & -0.632290000 & -0.326658000 \\
\hline $\mathrm{H}$ & 0.112724000 & -0.022808000 & -0.561244000 \\
\hline $\mathrm{H}$ & 2.846588000 & -0.507595000 & -1.182262000 \\
\hline 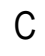 & 2.162054000 & -1.695651000 & 0.541365000 \\
\hline
\end{tabular}




\begin{tabular}{lrrr} 
O & 1.167938000 & -1.715230000 & 1.355048000 \\
O & 3.079862000 & -2.668723000 & 0.552573000 \\
$\mathrm{C}$ & 2.887623000 & -3.721812000 & 1.466241000 \\
$\mathrm{H}$ & 1.949943000 & -4.231038000 & 1.284982000 \\
$\mathrm{H}$ & 3.709968000 & -4.404690000 & 1.310805000 \\
$\mathrm{H}$ & 2.899341000 & -3.361895000 & 2.486701000 \\
$\mathrm{H}$ & 0.024692000 & 5.057914000 & 3.325313000 \\
$\mathrm{O}$ & -1.686282000 & 5.872610000 & 3.604590000 \\
$\mathrm{H}$ & -2.079938000 & 5.758008000 & 4.459979000 \\
$\mathrm{H}$ & -1.808540000 & 6.784475000 & 3.373995000 \\
$\mathrm{CI}-\mathrm{W} 2$ & & & \\
$\mathrm{C}$ & -2.366714000 & -0.858066000 & -0.067733000 \\
$\mathrm{C}$ & -1.110446000 & -1.344774000 & -0.293241000 \\
$\mathrm{C}$ & -0.048329000 & -0.456396000 & -0.634437000 \\
$\mathrm{C}$ & -0.303315000 & 0.934693000 & -0.712538000 \\
$\mathrm{C}$ & -1.563121000 & 1.426317000 & -0.499714000 \\
$\mathrm{C}$ & -2.588346000 & 0.537711000 & -0.174961000 \\
$\mathrm{H}$ & -3.179277000 & -1.505900000 & 0.207952000 \\
$\mathrm{H}$ & -0.898135000 & -2.389595000 & -0.179553000 \\
$\mathrm{H}$ & 0.531316000 & 1.565671000 & -0.942382000 \\
$\mathrm{H}$ & -1.788402000 & 2.473354000 & -0.556797000 \\
$\mathrm{O}$ & -3.782222000 & 1.050160000 & 0.034184000 \\
$\mathrm{C}$ & 1.236820000 & -0.940689000 & -0.842662000 \\
$\mathrm{C}$ & 2.425199000 & -0.140627000 & -1.078775000 \\
$\mathrm{H}$ & 1.281716000 & -2.025283000 & -0.781010000 \\
$\mathrm{H}$ & 2.855456000 & -0.152153000 & -2.062610000 \\
$\mathrm{C}$ & 3.177608000 & -0.127245000 & 0.057964000 \\
$\mathrm{O}$ & 2.772596000 & -0.506783000 & 1.183505000 \\
$\mathrm{O}$ & 4.436682000 & 0.358942000 & -0.049077000 \\
$\mathrm{H}$ & 5.183414000 & 0.447926000 & 1.132672000 \\
$\mathrm{H}$ & 5.335232000 & -0.524056000 & 1.583868000 \\
$\mathrm{H}$ & 6.139739000 & 0.868385000 & 0.849524000 \\
$\mathrm{H}$ & 4.705562000 & 1.091848000 & 1.859414000 \\
$\mathrm{H}$ & -4.425109000 & 0.391920000 & 0.268334000 \\
$\mathrm{H}$ & 0.997500000 & -2.574959000 & 1.776997000 \\
$\mathrm{H}$ & 1.480823000 & -3.234799000 & 2.254204000 \\
$\mathrm{H}$ & -1.837233000 & 1.683885000 \\
\hline
\end{tabular}

